\title{
Development of Concussion Evaluation Tools Using Life-Like Virtual Reality Environments
}

\author{
by \\ Yousef Sawires
}

\author{
A thesis \\ presented to the University of Waterloo \\ in fulfillment of the \\ thesis requirement for the degree of \\ Master's of Applied Science \\ in \\ Electrical Engineering
}

Waterloo, Ontario, Canada, 2019

(C) Yousef Sawires 2019 
I hereby declare that I am the sole author of this thesis. This is a true copy of the thesis, including any required final revisions, as accepted by my examiners.

I understand that my thesis may be made electronically available to the public. 


\begin{abstract}
Concussions are a common type of traumatic brain injury that can have severe consequences and are often induced by sports injuries. Once concussed, patients are more likely to sustain a second concussion, as they could be returned to play before they have fully recovered, in what is known as second-impact syndrome. Repeated concussions can also lead to delayed post-traumatic brain degradation, causing dementia and movement disorders. It is important to be able to detect concussions in an accurate and timely manner to avoid severe long-term effects. Current methods of diagnosis require a knowledgeable physician to be able to identify inconspicuous symptoms such as dizziness, headaches, nausea, and imbalance. It is necessary to develop new tools that enable the diagnosis of concussions reliably and quantitatively.

The researchers in this thesis propose a method to classify concussions in hockey players based on the reaction time to an external stimulus in a Virtual Reality (VR) environment. Different hockey scenarios were recorded with the use of a 360 degree camera and ambisonic microphone. The scenarios involved the dual-task challenge of a puck being passed towards the camera followed by a hockey player charging towards the camera. The collected audio and video are played back to participants on an Oculus Rift head-mounted display. The participants then attempt to track the puck and avoid the charging hockey player while the VR scenario is replayed. Electromyography (EMG) data is collected from the quadriceps femoris muscle group, minus the vastus lateralis, to measure the reaction time of the participants to the virtual collision. Motion capture data is also collected to track head directionality during the experimental procedure to gauge the spatial awareness of participants.

There appear to be no observable differences between participants with a history of concussions and participants without a history of concussions. Preliminary results will be shown; however, more data collection is needed in the future to be able to classify concussions based on reaction time and motion. In the future, this work will be expanded to players who have suffered a recent concussion ( $<1$ month), where their reaction times and motion will also be collected throughout their recovery.
\end{abstract}




\section{Acknowledgements}

I would like to thank my advisor Professor David Wang for his guidance, advice and feedback. I would also like to thank my research group, particularly Adam Gomes, Keegan Fernandes, and Elaine Huang for the continued help and support they have provided throughout this journey. Data collection would not have been possible without Monisha Naik, Elaine Huang, Madeleine Wang, Adam Gomes, and Keegan Fernandes.

This research would not have been possible without the funding and support provided by Key Rehab Inc. Special thanks to Scott McIlwraith, David Gonzalez, and Jean Taylor for all the help they have provided, especially with participant recruitment. Special thanks to David Gonzalez for his guidance.

Thanks to my readers, James Tung and Siby Samuel for reviewing and providing feedback on this thesis.

Finally I would like to thank my friends and family for their constant support and encouragement. I would like to thank my parents, Ehab Sawires, and Terez Ramzy for everything. Their love and support knows no bounds and I would never be able to reciprocate it. I would like to thank my sister, Mariam Sawires, and brother-in-law, Joey Youssef for their incredible love and support. I would like to thank Andrew Yacoub and Ebraam Yacoub for providing me with a second home whenever I needed help, love, or support. I would also like to thank Moe Samaha, Abdullah Ibrahim, Daniel Modaour, Nasser Naoum, Mahmoud Al-Samouly, and William El-Khazen for providing much needed humour and laughter. Lastly, I would like to thank the love of my life Nervana Wadie for her constant love and support, as well as for staying up countless nights to help me think and work through problems. 


\section{Dedication}

This is dedicated to my family and friends. 


\section{Table of Contents}

List of Tables $\quad$ ix

List of Figures $\quad$ x

Abbreviations $\quad$ xiii

1 Introduction $\quad 1$

2 Background $\quad 6$

2.1 Concussion Diagnostic Tools . . . . . . . . . . . . . . . . . 6

2.1.1 Sport Concussion Assessment Tool (SCAT) . . . . . . . . . . 7

2.1.2 Military Acute Concussion Evaluation . . . . . . . . . . . . . 10

2.1.3 Automated Neuropsychological Assessment Metrics . . . . . . . . . 10

2.1.4 Banyan Brain Trauma Indicator . . . . . . . . . . . . . . . . 11

2.1.5 Assessing Concussions using VR . . . . . . . . . . . . . . 11

2.2 Situational Awareness . . . . . . . . . . . . . . . . . . 12

2.2.1 Factors That Affect EMG Measurements . . . . . . . . . . . . . . 14

3 Methodology 16

3.1 IR Markers \& Situational Awareness . . . . . . . . . . . . . . . 16

3.2 Anatomy Background \& EMG . . . . . . . . . . . . . . 18

3.2.1 Appendicular Muscles . . . . . . . . . . . . . 18 
3.3 General Procedure . . . . . . . . . . . . . . . . . . . . . . 21

3.3.1 Overview of VR Simulation . . . . . . . . . . . . . . 22

3.4 Raw Data . . . . . . . . . . . . . . . . . . . . . . . . 32

4 Results $\quad 36$

4.1 Signal Analysis Overview . . . . . . . . . . . . . . . . . . 36

4.1 .1 Fourier Series . . . . . . . . . . . . . . 36

4.1 .2 Fourier Transforms . . . . . . . . . . . . . . 37

4.1.3 Short Time Fourier Transform Resolution Issues . . . . . . . . . . 37

4.1.4 Wavelet Transforms . . . . . . . . . . . . . . . . . 40

4.2 EMG Post-Processing . . . . . . . . . . . . . . . . . . . . . . . 43

4.2.1 Discrete Wavelet Transform . . . . . . . . . . . . . . . 44

4.2 .2 Continuous Wavelet Transforms . . . . . . . . . . . . . . 50

4.2 .3 Principal Component Analysis . . . . . . . . . . . . . . . . 54

4.3 Summary of Results . . . . . . . . . . . . . . . . . . . . 57

$\begin{array}{lll}5 & \text { Conclusions } & 65\end{array}$

6 Future Work \& Current Limitations $\quad 68$

6.1 Participant Data Collection . . . . . . . . . . . . . . . . 68

6.2 Wireless Approach . . . . . . . . . . . . . . . . . . . . . 68

6.3 EMG Improvements _ . . . . . . . . . . . . . . . . . . . . . 69

6.4 ECG and EEG Data Collection . . . . . . . . . . . . . . . . . . 69

6.5 Improved Motion Capture Technology _ . . . . . . . . . . . . . . 70

6.6 Theoretical Improvements _ . . . . . . . . . . . . . . . . . 70

6.7 Scenario Improvements . . . . . . . . . . . . . . . . . . 70

$\begin{array}{ll}\text { References } & 72\end{array}$

$\begin{array}{lr}\text { APPENDICES } & 80\end{array}$ 
$\begin{array}{ll}\text { A All the Different Hockey Scenes } & 81\end{array}$

A.1 Scenario 1 . . . . . . . . . . . . . . . . . . . 81

A.2 Scenario $2 \ldots \ldots \ldots \ldots$

A.3 Scenario $3 \ldots \ldots \ldots$. . . . . . . . . . . . . . . 85

A.4 Scenario $4 \ldots \ldots \ldots 8$

A.5 Scenario 5 . . . . . . . . . . . . . . . . . . 90

A.6 Scenario $6 \ldots \ldots \ldots \ldots$. . . . . . . . . . . . . . . . . . . . . . . . . .

A.7 Scenario $7 \ldots \ldots \ldots$. . . . . . . . . . . . . . . 94

$\begin{array}{ll}\text { B Survey Questions and Ethics Documents } & 97\end{array}$

B.1 SCAT Survey . . . . . . . . . . . . . . . . . . . 98

B.2 ACE Survey . . . . . . . . . . . . . . . . . . . 102

B.3 Data Collection Form . . . . . . . . . . . . . . . . . . . . 110 


\section{List of Tables}

1.1 Return to Play Protocol $[33] . \ldots \ldots \ldots \ldots$. . . . . . . . . . . . 2 


\section{List of Figures}

1.1 Experimental Setup with Cameras Circled in Yellow and IR Markers in Green 4

2.1 Flowchart of the Immediate Actions performed during a SCAT Examination $[23] \ldots \ldots \ldots \ldots \ldots \ldots$

2.2 Flowchart of the Cognitive Screening as part of the SAC [23] . . . . . . . 9

2.3 Model of Situational Awareness in Dynamic Decision Making [20]. . . . . . 13

3.1 Oculus Rift with Infrared (IR) Markers. . . . . . . . . . . . . . . . 17

3.2 Appendicular Muscles of the Lower Limb [42] . . . . . . . . . . . . . . . 18

3.3 Electrode Placement on Appendicular Muscles. . . . . . . . . . . . . . . . 20

3.4 Scenario $1 \ldots \ldots \ldots \ldots$

3.5 Scenario $2 \ldots \ldots \ldots \ldots \ldots$

3.6 Scenarios 3 and $5 \ldots \ldots \ldots \ldots$. . . . . . . . . . . . . . . . 24

3.7 Scenario $4 \ldots \ldots \ldots \ldots \ldots$

3.8 Scenarios 6 and $7 \ldots \ldots \ldots \ldots \ldots$

3.9 Real footage of Scene 8 from Scenario 2 . . . . . . . . . . . . . . 27

3.10 Participant with Skates on Synthetic Ice . . . . . . . . . . . . . . . . . 28

3.11 Real Time Visualization of Head Markers in Optitrack . . . . . . . . . . . 29

3.12 Communication between Different Components in the Experimental Procedure 30

3.13 OpenBCI EMG Data Streaming . . . . . . . . . . . . . . . . . 31

3.14 Pain Scale. . . . . . . . . . . . . . . . . . . . 32 
3.15 Raw EMG Data of the Right Rectus Femoris . . . . . . . . . . . . . . 33

3.16 Head Directionality from a non-concussed Participant . . . . . . . . . . . 34

3.17 Head Directionality from a Concussed Participant . . . . . . . . . . . . 35

4.1 Short Time Fourier Transform (STFT) with different Window Sizes . . . . 39

4.2 Morlet wavelets with different dilation and translation factors . . . . . . . 40

4.3 Wavelet Transform of Equation 4.5 . . . . . . . . . . . . . . . . 42

4.4 Post-processing Technique . . . . . . . . . . . . . . . . . . . . . 43

4.5 Mallat's cascaded filter $[65] \ldots \ldots \ldots$. . . . . . . . . . . 44

4.6 Daubechies 7 th Order Wavelet . . . . . . . . . . . . . . . . 46

4.7 db7 Decomposition and Reconstruction of the Scaling and Wavelet Functions 47

4.8 EMG Decomposition . . . . . . . . . . . . . . . . . . . . . . . . . 48

4.9 Denoised EMG Signal in Black, Original in Red . . . . . . . . . . . . . 49

4.10 Reaction Time Visualization . . . . . . . . . . . . . . . . 50

4.11 Effects of symmetry and time-bandwidth values on the shape a Morse wavelet 52

4.12 Continuous Wavelet Transform (CWT) of an EMG Signal for a Non-concussed Participant . . . . . . . . . . . . . . . . 53

4.13 CWT of an EMG Signal for a Concussed Participant . . . . . . . . . . 54

4.14 Principal Component Analysis (PCA) Example . . . . . . . . . . . 56

4.15 PCA for the EMG Data Set . . . . . . . . . . . . . . . . . 59

4.16 Principal Component 1 Boxplot . . . . . . . . . . . . . . . 60

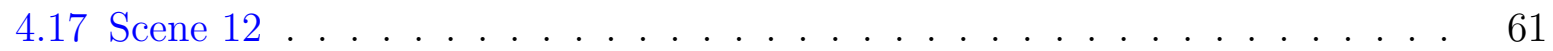

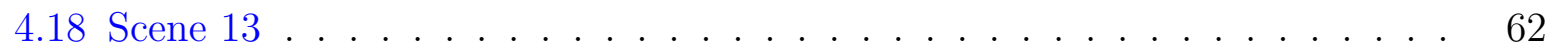

4.19 Scene 12 responses . . . . . . . . . . . . . . . . . . . . . 63

4.20 Scenes 12 and 13 Boxplot . . . . . . . . . . . . . . . 64

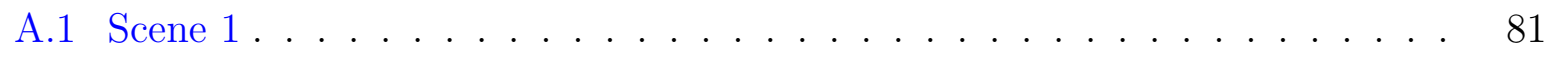

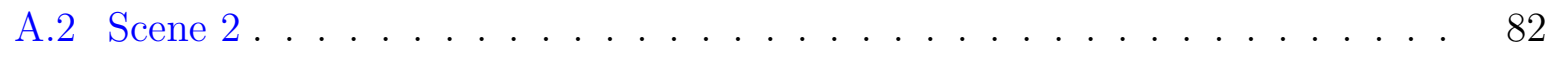

A.3 Scene $3 \ldots \ldots \ldots \ldots \ldots$. . . . . . . . . . . . . . . . . . . . 82 
A.4 Scene $4 \ldots \ldots \ldots \ldots$

A.5 Scene $5 \ldots \ldots \ldots \ldots \ldots$

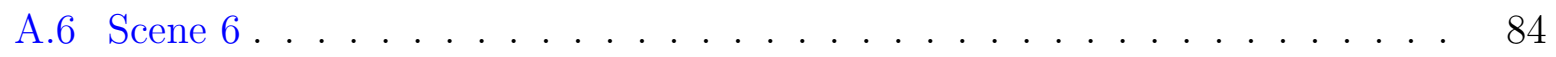

A.7 Scene $7 \ldots \ldots \ldots \ldots \ldots \ldots$

A.8 Scene $8 \ldots \ldots \ldots \ldots \ldots \ldots$

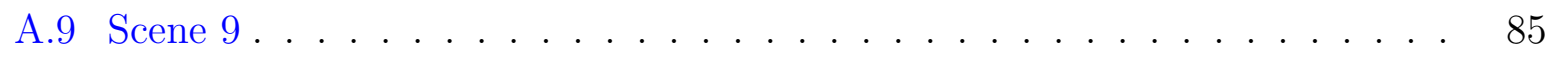

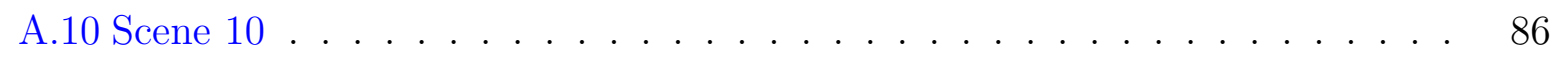

A.11 Scene $11 \ldots \ldots \ldots \ldots$

A.12 Scene $12 \ldots \ldots \ldots \ldots \ldots \ldots$

A.13 Scene $13 \ldots \ldots \ldots \ldots \ldots \ldots$

A.14 Scene $14 \ldots \ldots \ldots \ldots \ldots$

A.15 Scene $15 \ldots \ldots \ldots \ldots \ldots$

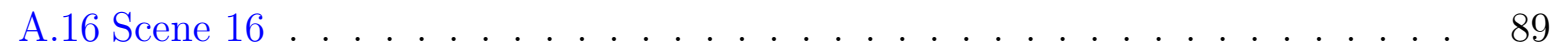

A.17 Scene $17 \ldots \ldots \ldots \ldots$

A.18 Scene $18 \ldots \ldots \ldots \ldots \ldots$

A.19 Scene $19 \ldots \ldots \ldots \ldots$. . . . . . . . . . . . . . . . . . . 90

A.20 Scene $20 \ldots \ldots \ldots \ldots$

A.21 Scene $21 \ldots \ldots \ldots \ldots$

A.22 Scene $22 \ldots \ldots \ldots \ldots$

A.23 Scene $23 \ldots \ldots \ldots$

A.24 Scene $24 \ldots \ldots \ldots$

A.25 Scene $25 \ldots \ldots \ldots$

A.26 Scene $26 \ldots \ldots \ldots \ldots$

A.27 Scene $27 \ldots \ldots \ldots$. . . . . . . . . . . . . . . . . . . . . . . . . . 94

A.28 Scene $28 \ldots \ldots \ldots$

A.29 Scene $29 \ldots \ldots \ldots$

A.30 Scene $30 \ldots \ldots \ldots \ldots$ 


\section{Abbreviations}

ACE Acute Concussion Evaluation 67

ANAM Automated Neuropsychological Assessment Metrics 10

BTI Brain Trauma Indicator 11

COM Centre of Mass 2, 3, 29

CT Computed Tomography 6

CWT Continuous Wavelet Transform xi, 43, 53, 54, 67, 70

DDWT Daubechies Discrete Wavelet Transform 43

DWT Discrete Wavelet Transform 47, 70

EMG Electromyography iii, xi, 3-6, 14-16, 18, 29, 30, 43, 44, 46-49, 51-54, 59, 65, 68, 70,71

GCS Glasgow Coma Scale 8

IR Infrared x, 3, 16, 17, 28, 29, 69

MACE Military Acute Concussion Evaluation 10

mBESS Modified Balance Error Scoring System 9

MRI Magnetic Resonance Imaging 6

PCA Principal Component Analysis xi, 4, 5, 43, 54-57, 59, 65, 70 
ROM Range of Motion 8, 9

SA Situational Awareness 3, 12, 13, 16

SAC Standardized Assessment of Concussion 8

SAGAT Situation Awareness Global Assessment Technique 13, 14, 67

SCAT Sport Concussion Assessment Tool 3, 7, 9, 10, 67

STFT Short Time Fourier Transform xi, 37-41, 50

TBI Traumatic Brain Injury 2, 7, 8, 12

VIGIL-CPT Vigil Continuous Performance Test 11

VR Virtual Reality iii, 3, 11-13, 26, 65, 66 


\section{Chapter 1}

\section{Introduction}

Concussions are a common type of traumatic brain injury that can have severe consequences and occur frequently among athletes [1]. In contact sports such as ice-hockey, recurrence of concussions is an issue since concussions can occur without the loss of consciousness [31]. There were 5.8 concussions per 100 players per season recorded during the NHL regular season games between 1997 and 2004 and only 18\% of the instances resulted in loss of consciousness [1].

The high acceleration in these situations exerts rotational forces on the brain that result in bio-mechanical injury and a malfunctioning metabolism. Other symptoms of concussions include headaches, nausea, confusion, memory loss and fatigue [45].

Despite the alleviation of these symptoms, it is still not safe to assume that athletes should return to their active lifestyles. This is because it is possible that symptoms may return and worsen after physical or cognitive exercise either right after exercise or even the day after. There are step-wise return to play protocols that guide the athlete during rehabilitation where the athlete is required to be asymptomatic at each single level before progressing to the next step of the protocol [33]. An example of such protocol is shown in Table 1.1. 


\begin{tabular}{|l|l|}
\hline Step 1: & No activity, complete rest \\
\hline Step 2: & $\begin{array}{l}\text { Light aerobic exercise such as walking or stationary cycling, no } \\
\text { resistance training }\end{array}$ \\
\hline Step 3: & Sport-specific training \\
\hline Step 4: & Non-contact training drills \\
\hline Step 5: & Full contact training after medical clearance \\
\hline Step 6: & Game play \\
\hline
\end{tabular}

Table 1.1: Return to Play Protocol [33].

Table 1.1 is only applicable to simple concussion cases and might not be a suitable protocol for complex or recurring concussions [33]. In some instances, it is advised not to return to contact sports or collisions as there is proof that individuals who have suffered a concussion are more prone to suffering another [6, 63].

Beyond the aforementioned general symptoms, concussions impact the visual motor coordination [14], impede information processing [2], and affect gait stability [52]. Jordan et al. administered several neurocognitive tests to professional boxers and found decreased performance in visual motor coordination as sparring increased. These findings suggest that repetitive concussive and sub-concussive Traumatic Brain Injury (TBI) blows may result in the deterioration of cognitive brain functionality [30]. Similar cognitive defects were found in collegiate athletes participating in ice hockey [32]. The notion that there is complete recovery after a single mild head injury is also under scrutiny. In a test where the event related potential P300 wave is measured for a set of easy and difficult tasks, it was found that those who had suffered a mild head injury had a reduced P300 amplitude and performed more poorly than the controls group on cognitive tasks several years after injury. These results seem to point to impaired information processing speed and a struggle with divided attention [2]. Furthermore, a study measuring Centre of Mass (COM) sway found an a significant increase in imbalance while the subjects were engaged in simple cognitive activities, which confirms the hypothesis that a mild head trauma restricts the ability to divide attention across different tasks. Gait instability was also noticeable thought not as significant when attention was undivided [52].

It is important to be able to detect concussions in an accurate and timely manner to avoid severe long term effects. Once concussed, patients are more likely to sustain a second concussion as they could return to play before they have fully recovered in what is known as second-impact syndrome [7]. Repeated concussions can also lead to delayed 
post-traumatic brain degradation causing dementia and movement disorders [46]. Current methods of diagnosis require a knowledgeable physician to be able to identify inconspicuous symptoms such as dizziness, headaches, nausea, and imbalance. Additionally, diagnostic tools such as the Sport Concussion Assessment Tool (SCAT) are traditionally being used. This diagnosis consists of a questionnaire that helps identify 22 possible symptoms, with the presence of one symptom being sufficient for a diagnosis [25]. Unfortunately, these diagnostic tools rely on the patients' cooperation alongside several physical factors [10]. Such physical factors include sex [15, 22, 39, 62, 64, 69], education level [69], age [58], concussion history [5, 64], and depression and anxiety levels [58]. Concussed athletes may also hide their symptoms so that they can continue playing and avoid long recovery periods. Therefore, it is necessary to develop new tools that enable the diagnosis of concussions reliably and quantitatively.

The research in this thesis propose a quantitative method to diagnose and check the progression of a concussion. This involves using VR in conjunction with EMG responses and tracking the directionality of participant movements using a motion tracking system. The study will primarily focus on concussions sustained by hockey players, with the hopes of expanding to athletes of other sports in the future. Players will be immersed into a lifelike hockey environment using VR. To achieve the desired realism, a 360-degree camera and ambisonic microphone will be used to record real life hockey scenarios in a hockey rink, where players simulate intercepting passes as well as attempting to check a virtual player situated at the camera locator. Ambisonic microphones, unlike standard microphones, encapsulate several diaphragms for capturing full-spectrum sound from all directions. These recordings will then be inserted into a virtual world to perfectly recreate the desired situation with accurate 360 video and audio. It is important to include ambisonic recordings to recreate 360 audio, as this helps immerse the participant in a more realistic experience.

Electrodes will be attached to several muscles below the torso in order to measure muscle twitch activity and collect EMG data. Additionally, motion capture, via infrared cameras and markers placed on the posterior of the head, will be used to track the directionality of participants during dynamic motion to assess the Situational Awareness (SA) of the participants. VR based neuropsychological testing has been shown to have a high ecological validity and so is more applicable to real life settings [54], this enables us to create an environment in which the hockey players would be exposed to familiar and realistic conditions. This experimental setup can be seen in Figure 1.1 which captures a participant with skates on synthetic ice surrounded by two Optitrack cameras (circled in yellow) and six IR markers (three on the head and three on the waist, circled in green). Future investigations of Optitrack data can also allow us to analyze COM accelerations during gait, which is known to be an issue among concussed patients [52]. 


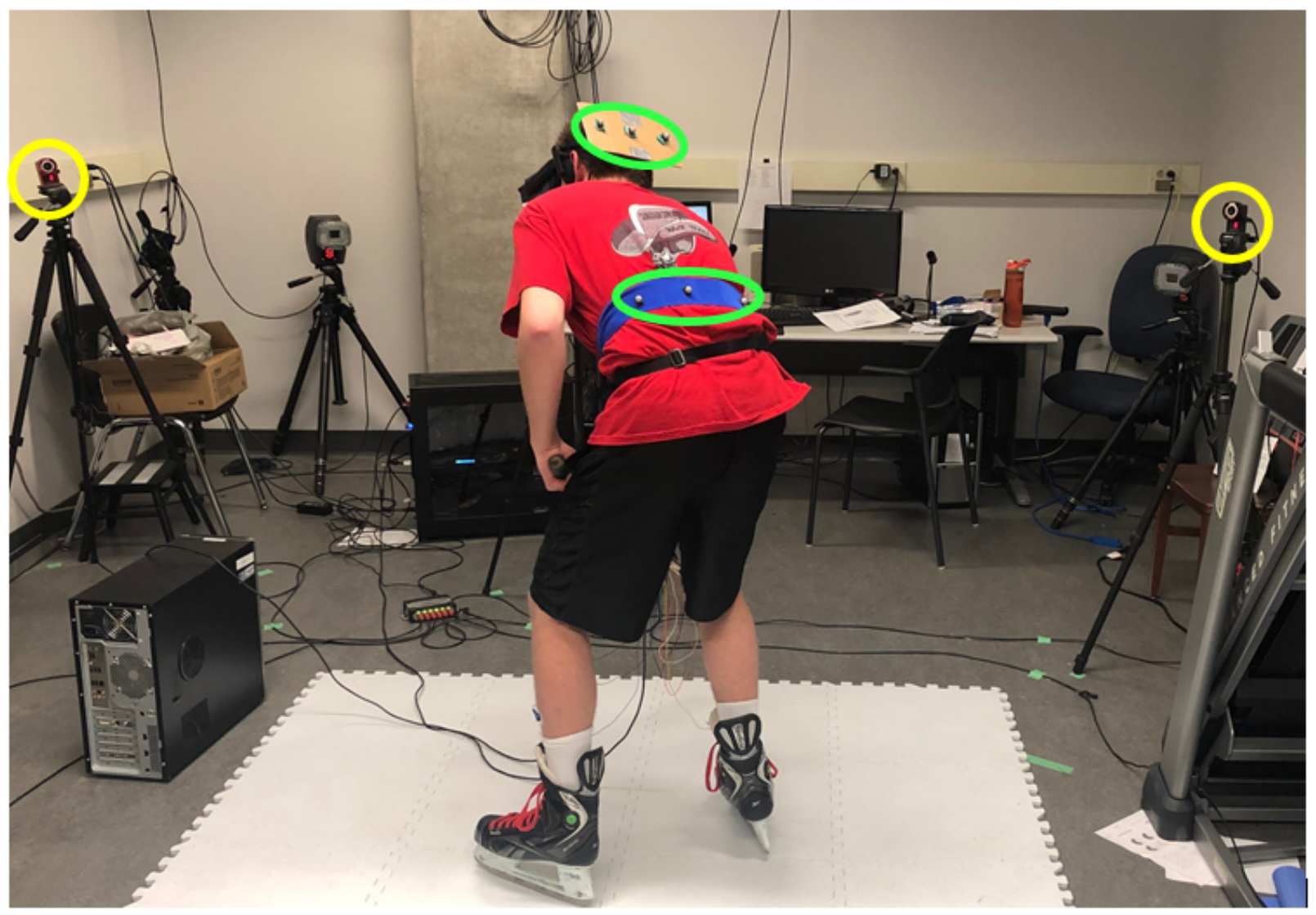

Figure 1.1: Experimental Setup with Cameras Circled in Yellow and IR Markers in Green

Once the data has been captured, signal processing methods will be incorporated to help analyze the data for statistical significance. The hypothesis is that concussed hockey players have slower reaction times than non-concussed hockey players. The researchers propose a method of analyzing EMG data using wavelet transforms [56]. Unlike Fourier transforms, wavelet transforms are localized in both time and frequency and can therefore be utilized to analyze a signal that varies in frequency over time. This representation should be visually intuitive to a physician since it can display muscle twitch activity over time and would help identify reaction time, which is one of the key markers for concussion diagnosis. Reaction times can be further processed using PCA, a pattern recognition algorithm which transforms the data to a new coordinate system based on the largest variances in the data set [28]. Classification of different groups (e.g. concussed vs non-concussed) with the use of PCA then creates a visual aid to help determine if there are any differences in reactions times. Boxplots are finally incorporated to encapsulate the new data after PCA analysis. 
Refer to Chapter 2 for more information regarding PCA implementation.

The chapters in the thesis are as follows. Chapter 2 reviews the background behind current concussion diagnosis tools as well as background concepts in signal analysis. Chapter 3 outlines the instrumentation used and describes the methodology carried out during the experimental procedure. Chapter 4 presents the results of both EMG and directionality data as well as the appropriate signal processing techniques. Chapter 5 draws conclusions on the presented results. Lastly, Chapter 6 gives insight into some of the weaknesses of the experimental procedure and outlines areas for improvement. Future research issues are also explored. 


\section{Chapter 2}

\section{Background}

In order to create concussion diagnostic tools to aid physicians and clinicians in their assessments, several research areas have arisen. Of most relevance are previous work in concussion diagnosis tools, and the physical effects of concussions, both symptomatic and non-symptomatic. This chapter provides the background information behind current-day concussion assessment tools along with their advantages and disadvantages, which will in turn provide a leeway into the advantages of the current research in this thesis. Also in this chapter, the physical effects of concussions will be reviewed alongside Endsley's Situational Awareness Model. Lastly, the anatomy of the lower limbs, specifically the appendicular muscles will be presented to familiarize the reader with the set of muscles used for EMG testing.

Previous work has been conducted on diagnosing the effects of concussions. The complexity arises in the elusiveness of the diagnosis since the use of traditional brain imaging techniques such as Computed Tomography (CT) scans and Magnetic Resonance Imaging (MRI) scans prove to be of no use in cases where there is no internal bleeding [72].

\subsection{Concussion Diagnostic Tools}

Several concussion assessment tools have been developed in the past to aid in the clinical evaluation of concussions. This thesis will consider the most widely used tools on the sidelines of major sports such as hockey, football, soccer and rugby. 


\subsubsection{Sport Concussion Assessment Tool (SCAT)}

The SCAT survey is an example of such a tool, and has been incorporated in this experiment to aid in the analysis. The SCAT3 is used in sideline assessments and is implemented by several sports associations such as FIFA, the Olympics, and IIHF [23].

Immediately after head impact, the physician assesses the state of the athlete and looks for any clear signs of trauma. This includes loss of consciousness, lack of coordination, disorientation/confusion, loss of memory, and any visible superficial impact on the head. There are also several tests within the SCAT3 assessment to evaluate the severity of the head trauma.

It is valuable to briefly explore the SCAT procedure, considering its use across major sporting events and the research in this thesis.

\section{SCAT Procedure}

The latest revision of the SCAT survey is the SCAT5 (2017) which was published 4 years after SCAT3. The latest edition synthesized the latest research, included additional tests and refined previous tests [19].

The SCAT survey is divided into 6 major sections, beginning with an immediate onfield assessment to identify any life-threatening injuries, and gradually following through

with standardized tests once it has been determined that the athlete is in no immediate danger.

\section{Section 1: Immediate Assessment}

The first step of the procedure after an athlete has suffered a TBI is for the sideline physicians to immediately evaluate the player and check for any "Red Flags". These involve (and are not limited to) any of the following symptoms:

1. Weakness or tingling in the arms or legs

2. Severe headache

3. Seizures or convulsions

4. Loss of consciousness 


\section{Vomiting}

If any of these symptoms are available, the athlete is in need of immediate medical attention and must carefully be transferred to the hospital.

If none of these immediate signs are noticeable then the clinicians will search for any observable signs such as disorientation, confusion, a blank or vacant countenance, and imbalance. This is followed by the "Memory Assessment Maddocks Questions" where a few simple questions are presented to the athlete in order to ensure that the athlete is not suffering from any memory loss [40]. The following step is to perform the Glasgow Coma Scale (GCS) examination, where a scored is assigned to the athlete's eye response, verbal response, and motor response. Finally, a Cervical Spine Assessment is performed to ensure that the athlete's neck has full Range of Motion (ROM) and is free from pain.

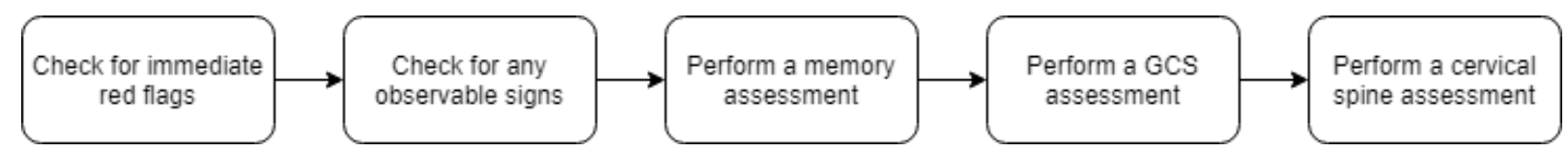

Figure 2.1: Flowchart of the Immediate Actions performed during a SCAT Examination [23]

Figure 2.1 summarizes the procedures performed by clinicians and physicians immediately after an athlete has suffered a TBI. Once this examination is complete, the physicians then move on to the next step where a series of tests are performed in an off-field assessment.

\section{Section 2: Off-field Assessment}

The off-field assessment is comprised of two tasks. Firstly, the background information of the athlete is collected, both personal and medical. This includes information such as history of concussions suffered and list of current medications. Secondly, the athlete is required to complete a symptom evaluation list on their own and check off the 22 symptoms on a severity scale. The symptom evaluation is completed twice, the first time to identify a baseline score based on how the athlete typically feels, and a second time post-injury.

\section{Section 3: Cognitive Screening}

For Section 3 of the examination, a Standardized Assessment of Concussion (SAC) test is performed [44]. The SAC is comprised of 4 different tests, where each response is given 
a score. The first test is an orientation test designed to test the athlete's ability to recall the date including the day, month, year alongside the time in hours. This is followed by an immediate memory test where a list of 5 words is read aloud to the athlete, who's then asked to recite the list backwards. The third test focuses on the concentration of the athlete who's then required to recite a string of numbers in reverse order. Lastly, the athlete is asked to recite the months of the year in reverse order.

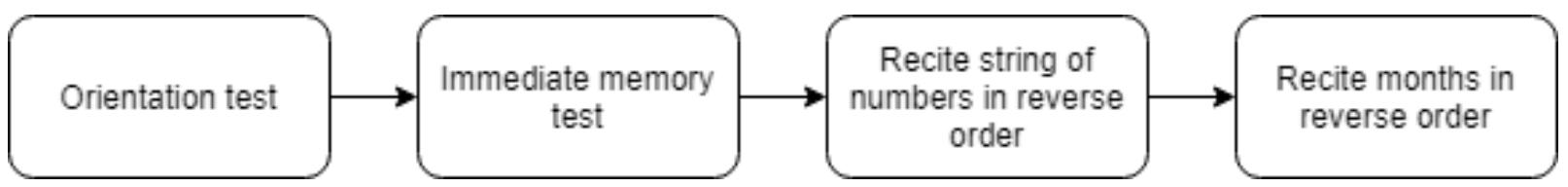

Figure 2.2: Flowchart of the Cognitive Screening as part of the SAC [23]

Figure 2.2 above displays a flowchart to summarize the testing performed during the cognitive screening aspect of the SCAT.

\section{Section 4: Neurological Screen}

Step 4 of the procedure tests the athlete for any observable neurological disorders and imbalances. The athlete is graded on their ability to follow instructions, display full cervical spine ROM without pain, perform the finger nose test, move their head and neck without experiencing double vision, and perform tandem gait normally [23].

Once the above has been graded, a Modified Balance Error Scoring System (mBESS) test is performed. This involves testing the athlete's ability to maintain balance in several different scenarios, where a grade for each is assigned.

\section{Section 5: Delayed Recall}

The delayed recall test involves reciting the words used in the immediate memory test in Section 2.1.1 after waiting 5 minutes from the end of the immediate recall section.

\section{Section 6: Decision}

The scores from all previous 5 sections are then tallied up and a decision is made regarding the status of the athlete. It is important to note that the SCAT is not intended to be used 
alone to diagnose concussions or inform the physician about the recovery time required before the athlete can return to play.

While the SCAT examination is widely used by several sporting associations it still contains several flaws. The SCAT method does not make use of any bio-markers for accurate diagnosis, which puts in question the accuracy of the testing involved. Furthermore, it should be noted that not all concussions display symptoms at the onset of impact. In fact, research performed on a sample of college athletes found that $50 \%$ of those who sustained concussions did not experience immediate symptomatic effects [13]. SCAT scores have also been found to vary with age and sex, the significance of which is not known.

One of the big issues with the SCAT examination is that it does not provide any insight into the recovery of the athlete, nor does it give any quantitative indication of the severity of the concussion. Therefore, unless a concussion has immediate significant symptomatic impact, it will not be identified by the SCAT examination.

\subsubsection{Military Acute Concussion Evaluation}

Similar to the SCAT examination the Military Acute Concussion Evaluation (MACE) examination is a survey with no bio-markers involved. Due to the absence of any reliable neurocognitive impairment test, the American military employs the use of MACE in the battlefield. Another disadvantage of the MACE is that the validity of the test decreases if the test is not administered immediately after head injury [13].

\subsubsection{Automated Neuropsychological Assessment Metrics}

Neurocognitive tests, the most widely used being the Automated Neuropsychological Assessment Metrics (ANAM), have also been developed to measure cognitive parameters such as attention, concentrations, and reaction time [9]. However, further investigation has questioned whether the ANAM test is accurate in long-term assessment of patients [12].

This thesis is interested in identifying all anthropometric variables that could affect the results, as well as identifying the validity of the process for long-term testing. 


\subsubsection{Banyan Brain Trauma Indicator}

The Banyan Brain Trauma Indicator (BTI) is the first blood test developed to test concussions in adults [51]. The principle is that two protein bio-markers are released by the brain into the blood stream within 12 hours of impact. However, there are several questions which need to be addressed. It's still unclear whether this indicator can be used to aid with recovery. It is also unclear how long the proteins remain in the bloodstream and whether the quantity of these proteins correlates to the severity of the concussion. While the initial findings look promising, the Banyan BTI seems to only confirm the presence of a head trauma.

\subsubsection{Assessing Concussions using VR}

The assessments of concussions using VR is a relatively new field of research with great potential due to the nature of VR being able to recreate a life-like immersive environment in which the participants can be assessed.

A study done in 2012 incorporated VR as a tool for assessing sports concussions in adolescents [49]. The experiment relies on the use of Vigil Continuous Performance Test (VIGIL-CPT) as a computerized test [8]. The participants are immersed in an environment where a letter appears on a whiteboard at different intervals, and a keyboard response is required of them whenever the letter appears. It was reported that the concussed group reported increased symptoms of cybersickness compared to the control group. Findings also suggest that sports concussions were associated with minor deficits in attention and concentration. While cybersickness is used as a measure for concussions, there is no quantifier indicating the severity of the concussion. There is also no indication of whether this can be used to indicate when participants can return to an active lifestyle. Furthermore, the VIGIL-CPT computerized test was initially developed to test concentration and attention in order to identify attention deficit issues in adolescents. There is no indication that using the VIGIL-CPT test in a VR environment has any added benefits.

More recently, the use of VR in detecting balance impairment following a mild traumatic brain injury has been shown to be valid and can replace expensive equipment. Furthermore, it has been shown to be useful in a rehabilitation setting by monitoring and controlling the participant's posture and balance [73].

The use of VR in the rehabilitation of stroke patients is greatly increasing as more data confirms the increased recovery of motor functions and daily activities [75]. This is promising as several research papers are embarking on the exploration of the use of 
VR in a rehabilitation and identification setting for concussions [3, 16-18, 61, 67, 73, 74]. However, no work has been done in classifying the severity of concussions and identifying an indicator that can be used to accurately assess the state of the athlete and determine their ability to return to sports.

\subsection{Situational Awareness}

One concept from Neuropsychology that is worth investigating for the purposes of this thesis is SA. SA is broken down into 3 distinct levels. Level 1 is the perception of all the elements in the environment within a volume of time and space. Level 2 is the integration of the different elements and the comprehension of the current situation, and Level 3 is the prediction of their states in the near future [20].

Concussions can have a drastic impact on an individual's SA often in the form of a lack of perception of dangerous elements in the environment, or a delayed response in evading them. A study conducted in 2010 found that drivers were late in responding to important cues when driving after they had suffered a mild TBI [57].

Figure 2.3 provides an overview of Endsley's SA model. This will prove to be useful during the research for this thesis since it will give insight into which specific area of cognition has been affected to help determine the severity of the concussion. 


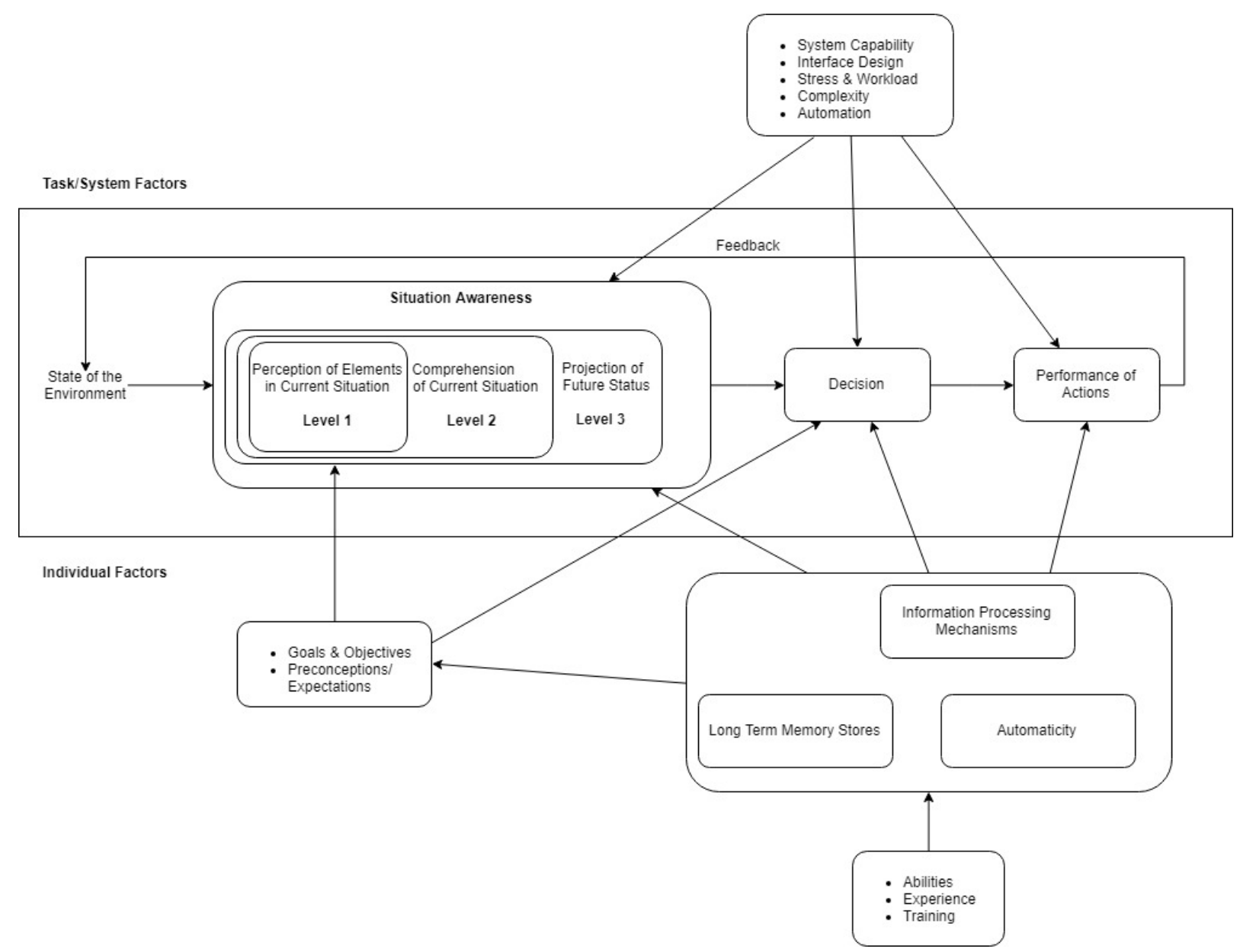

Figure 2.3: Model of Situational Awareness in Dynamic Decision Making [20].

Loss of SA has drastic effects as determined by Endsley. There are three main components that can gauge the severity of SA loss and which have been utilized in the experimental design. First, spatial disorientation can be worsened by the use of VR [48]. Second, a lack of system awareness can occur which encompasses insufficient scanning of the surrounding. Last, a lack of task awareness can exist where competing tasks are not handled properly.

Situation Awareness Global Assessment Technique (SAGAT) is a query-based measure used to assess SA by asking the participants a set of predetermined questions during any experiment. It is informally utilized during the experimental procedure in this thesis to ensure that the participants are not experiencing drastic disorientation and that they're 
aware of the dual-task of receiving the puck and bracing for a body-check. However, the use of SAGAT is limited since it introduces the challenge of being intrusive and can affect performance.

\subsubsection{Factors That Affect EMG Measurements}

Measuring EMG with surface electrodes is tricky due to 3 factors that can make the procedure quite challenging. These factors will be carefully identified and minimized based on previous guidelines and research [59].

\section{Causative Factors}

Causative factors are generally subdivided into two distinct classes, Extrinsic and Intrinsic factors. Extrinsic factors include electrode placement, the shape of the electrode, the medium between the electrode and the contact surface, and the location of the electrode with respect to the belly of the muscle. These factors are generally minimized by ensuring that the surface is smooth, which involves shaving any hair to remove any source of interference. Furthermore, having one researcher consistently place the electrodes on all the participants helps reduce the variability of the placement of the electrodes.

The intrinsic factors comprise anatomical and physiological factors that are determined by the number of active motor units such as blood flow, and fiber diameter. It is beyond the scope of this thesis and research to determine and account for these variables.

\section{Intermediate Factors}

Intermediate factors are physiological phenomena brought about by causative factors. Most notably, crosstalk from neighbouring muscles cause intermediate factors and reduce the reliability of the EMG data. This is even more pronounced in the case where dynamic movement is involved, which makes EMG data more susceptible to crosstalk from other muscles. The research involved in this thesis attempted to mitigate these errors by examining larger muscles below the torso that are less influenced by crosstalk.

\section{Deterministic Factors}

Deterministic factors are the direct results of the number of motor firing rate and the interaction between different muscle fibers. It is important to note that these factors have 
a direct effect on the information in the EMG signal; however, they are outside of the scope of the research in this thesis. 


\section{Chapter 3}

\section{Methodology}

The use of IR markers is described in this chapter by considering SA and Endsley's model. Furthermore, an outline of the Appendicular muscles is shown in this chapter in order to familiarize the reader with the anatomical structure of the leg muscles, which is crucial for understanding where the EMG electrodes should be placed. The last section of this chapter is focused on the procedural aspect of the experiment.

\subsection{IR Markers \& Situational Awareness}

SA plays a big role in hockey games since players are required to process a vast amount of information, and predict future events. Tracking the participants' directionality during the experiment gives insight into the level of SA that each participant is capable of. A participant with significant experience, who is not capable of processing the number of players in the rink, the location of the puck, and their own location in the rink may have suffered a severe concussion that has impacted Level 1 and Level 2 of their SA. A participant that is aware of their surroundings but is slow to determine which of the opposing players will body-check them may have suffered a milder concussion that has impacted Level 3 of their SA.

IR markers placed on the back of the participants' heads will allow for gathering directionality data that can be used to track where in the simulation the participants are facing, and whether they're aware of the location of the puck and the opposing virtual player who will body-check them. The setup of the Oculus Rift with IR markers can be seen in Figure 3.1 . 


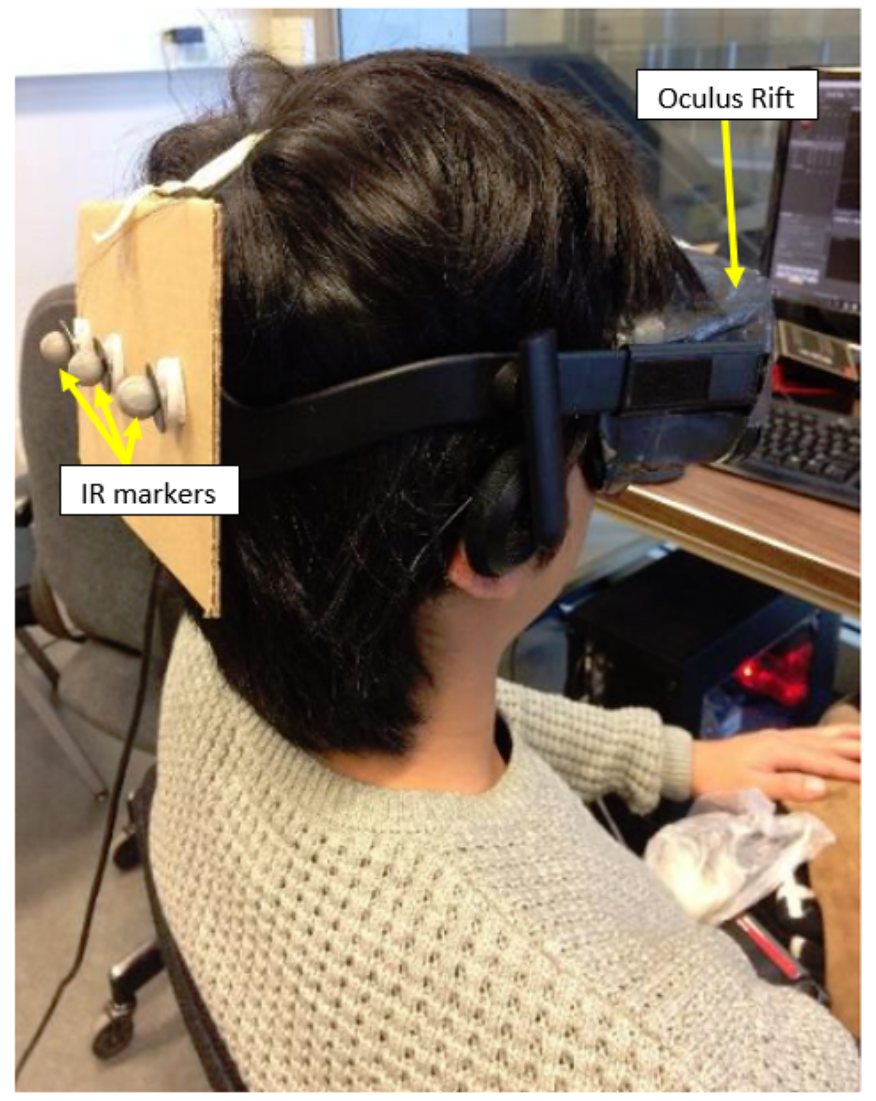

Figure 3.1: Oculus Rift with IR Markers.

Optitrack is chosen for research purposes as a result of the low noise levels as compared to other types of motion capture solutions such as inertial and regular computer vision [27]. IR markers allow for quick modifications to experimental design and have shown to be robust throughout dynamic movement when attached properly to the desired element. It is worth noting that Optitrack does experience drift with longer duration of testing (30 minutes), which will not impact the data in this thesis as the experimental duration is at most 15 minutes. Refer to Chapter 6 for improvements to the motion capture system. 


\subsection{Anatomy Background \& EMG}

Recall from Chapter 1 that electrodes are placed on specific leg muscles in order to measure the participants' reaction time through EMG measurements during the experiment. It is important to note where the EMG electrodes are placed and the justification behind their location.

\subsubsection{Appendicular Muscles}

There are 8 surface EMG electrodes that are placed on each participant. Figure 3.2 below displays the 4 muscles and 2 bones where the electrodes are attached.

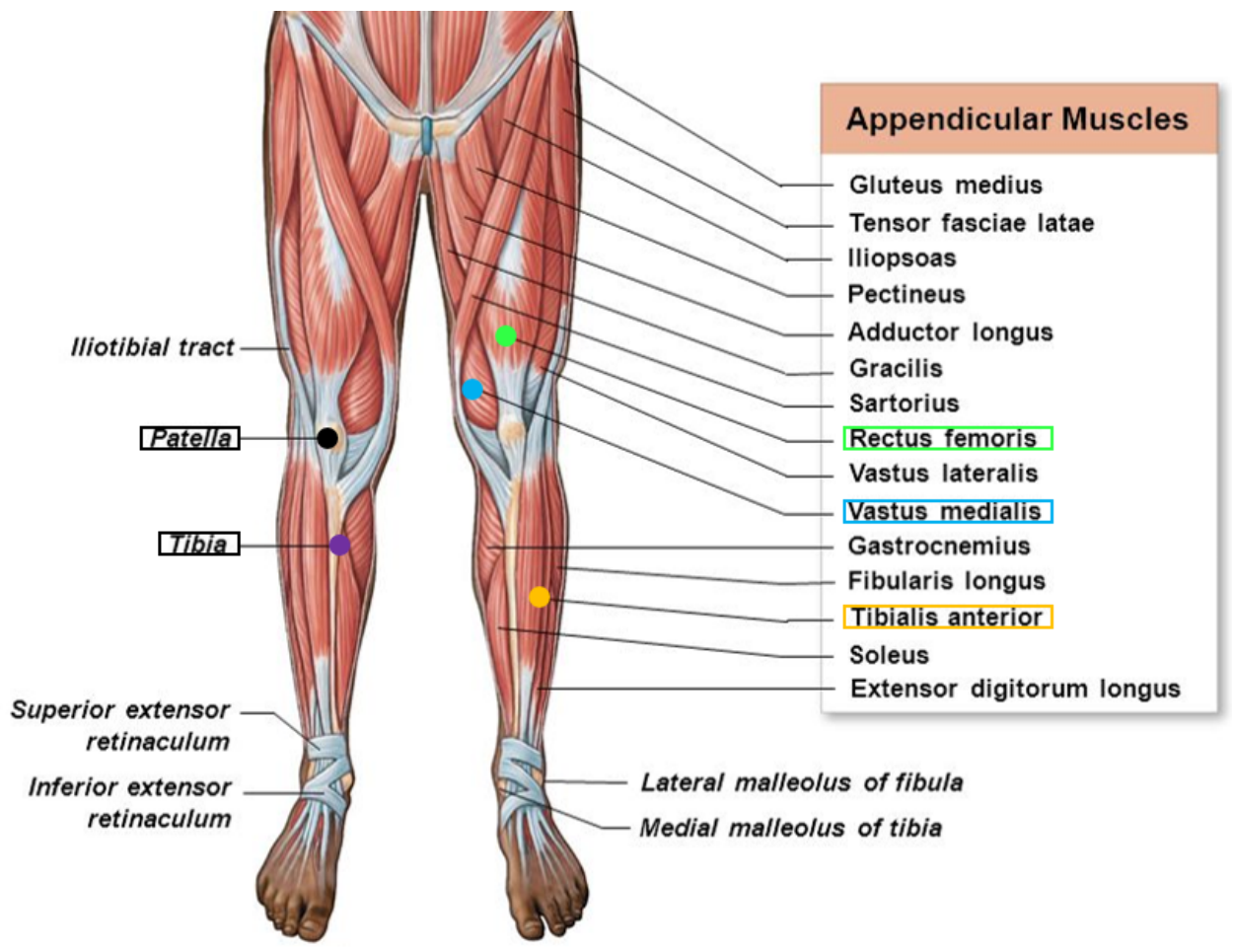

Figure 3.2: Appendicular Muscles of the Lower Limb [42].

More specifically, eight disposable surface electrodes are placed on the participants legs on the: 
1. Right Tibial Tubercle

2. Right Patella

3. Right Rectus Femoris

4. Right Vastus Medialis

5. Right Tibialis Anterior

6. Left Rectus Femoris

7. Left Vastus Medialis

8. Left Tibialis Anterior

Figure 3.3 demonstrates the placement of the electrodes on a participant during the experimental procedure. An elastic bandage wrap was initially used for cable management as can also be seen in the figure; however, it proved to be a source of interference. 


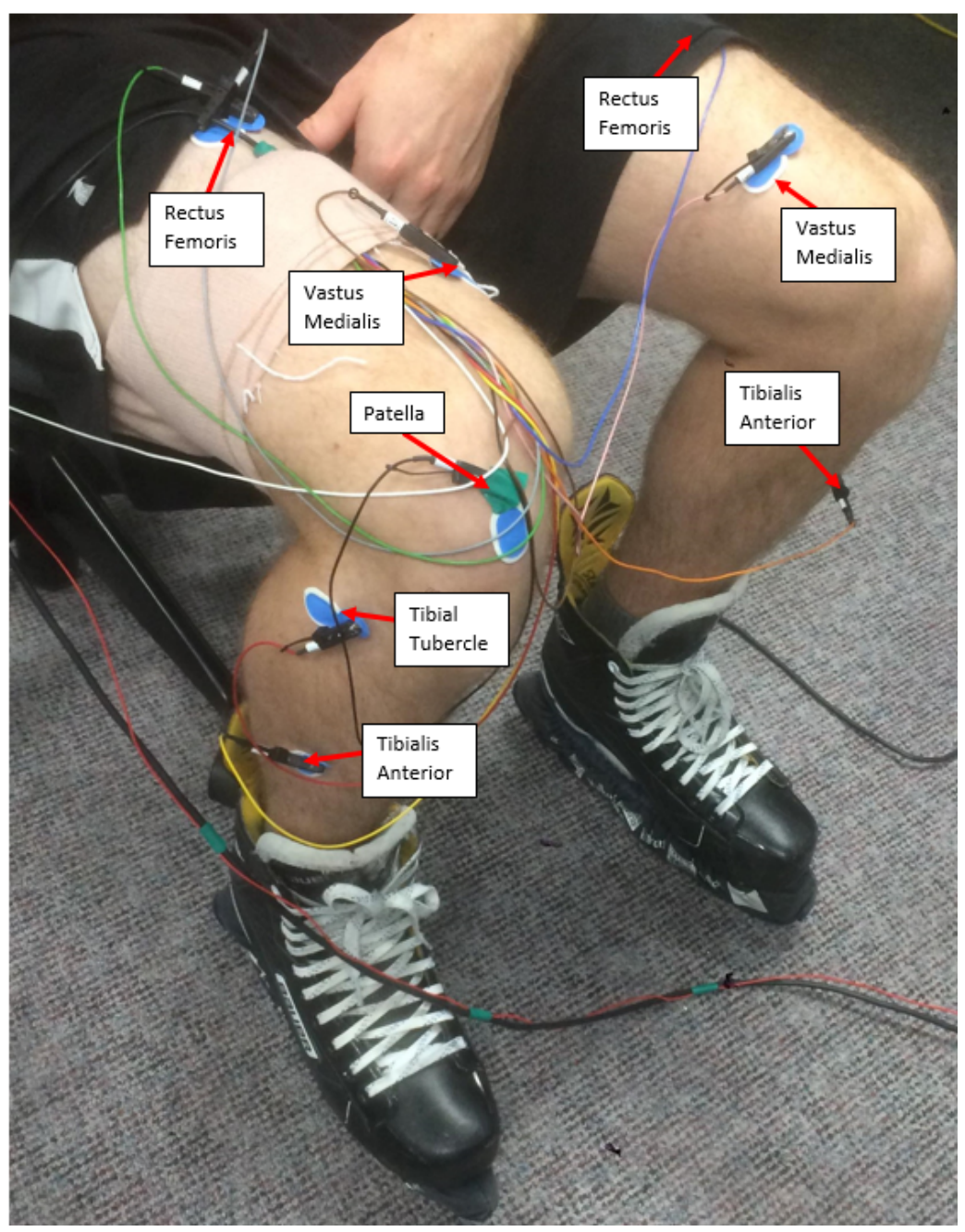

Figure 3.3: Electrode Placement on Appendicular Muscles.

The reason behind placing the electrodes in the specified locations has to do with the nature of the electrodes used and the muscles that the electrodes are placed on.

During dynamic movement such as those in hockey and for ethical reasons, it is not possible to insert indwelling electrodes directly into the muscle. The only solution is to utilize surface electrodes which are not as accurate or reliable [66]. In order to minimize the effects of the inaccuracies brought about by the surface electrodes, it is important to use larger muscles to take measurements since it reduces the possibility of muscle cross-talk. The Rectus Femoris, Vastus Medialis, and Tibialis Anterior have all shown in previous 
research to be reliable when measured using surface electrodes [43, 47, 59].

These muscles are part of the Quadriceps Femoris muscle group and form a complete set when the Vastus Lateralis is included [4]. Collectively, the quadriceps femoris muscle group act as the main extensors of the leg at the knee joint. However, due to limitations in the number of EMG channels, several muscles were not taken into account including the Vastus Lateralis and any of the posterior muscles such as the Gastrocnemius, and as a result, flexion is not well covered.

Despite the limitations in the number of muscles that could be measured, the chosen muscle group provides excellent insight into some of the most active muscles during icehockey [26].

\subsection{General Procedure}

This section describes the general procedure undertaken to carry out the experiment.

An invitation to participate in the experiment is sent out to several intramural icehockey teams at the University of Waterloo. An ethics approved information letter is also sent out detailing the experimental procedure, the exclusion criteria, and the risks and benefits. Participants who are interested, and who qualify for the experiment are then scheduled to undergo the experiment.

The participants are told in advance to wear shorts, a tight fitting shirt, and to bring their hockey skates. The first step of the procedure is for the participants to fill out the ACE and SCAT surveys, which have been detailed in Chapter 2. Upon completion, the participants are required to fill out a Consent Form and a Data Collection Form. The Data Collection Form records the following parameters:

- Age

- Gender

- Height

- Weight

- Level of Play

- Contact/Non-Contact Play 
- Level of Experience

- History of Concussions and Treatment

- Dominant Hand

The participants are then prepped for the experiment. This involves shaving any hair on the location where the electrodes are placed to minimize any interference. Once they are in proper clothing, with skates on, the experiment commences.

\subsubsection{Overview of VR Simulation}

After discussions with physiotherapists who are familiar with concussions brought about by sports, specifically hockey, several scenarios on the ice rink were devised. The focus was on scenarios where a concussed individual's lack of spatial awareness could result in physical contact that could lead to injury or, head trauma. Equipped with a 360 degree video camera and an ambisonic microphone to capture 360 degree audio, 30 scenes were created in order to test the participants' spatial awareness and stability. The goal is to create an immersive life-like experience of real hockey scenarios in a risk-free environment that can allow the researchers to collect data. The 30 scenes are divided across 7 different scenarios. The scenarios are similar in their setup but differ in where the camera is placed in the ice rink, and the number of players involved in the recording. 


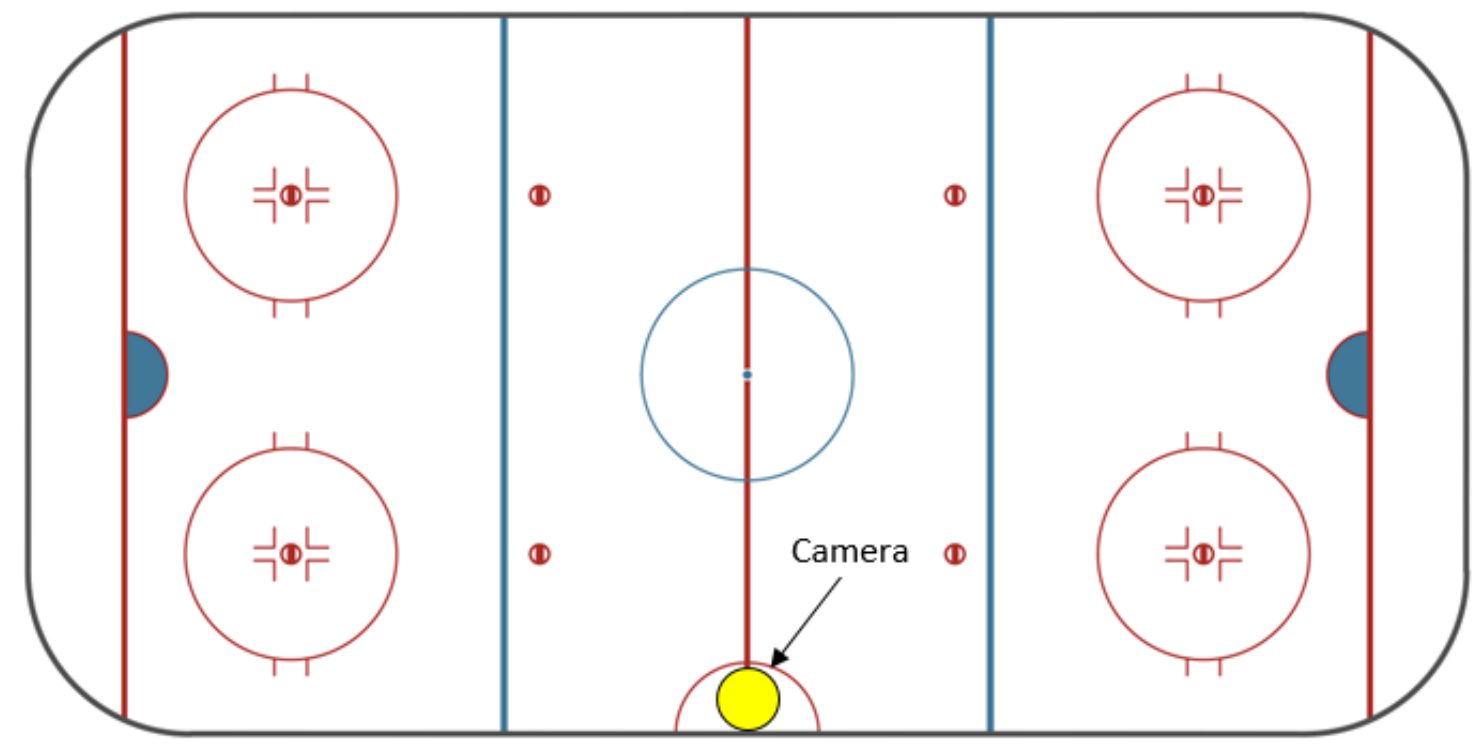

Figure 3.4: Scenario 1

In Figure 3.4 the camera is placed in the referee crease. Scenes 1-4 are carried out in scenario 1.

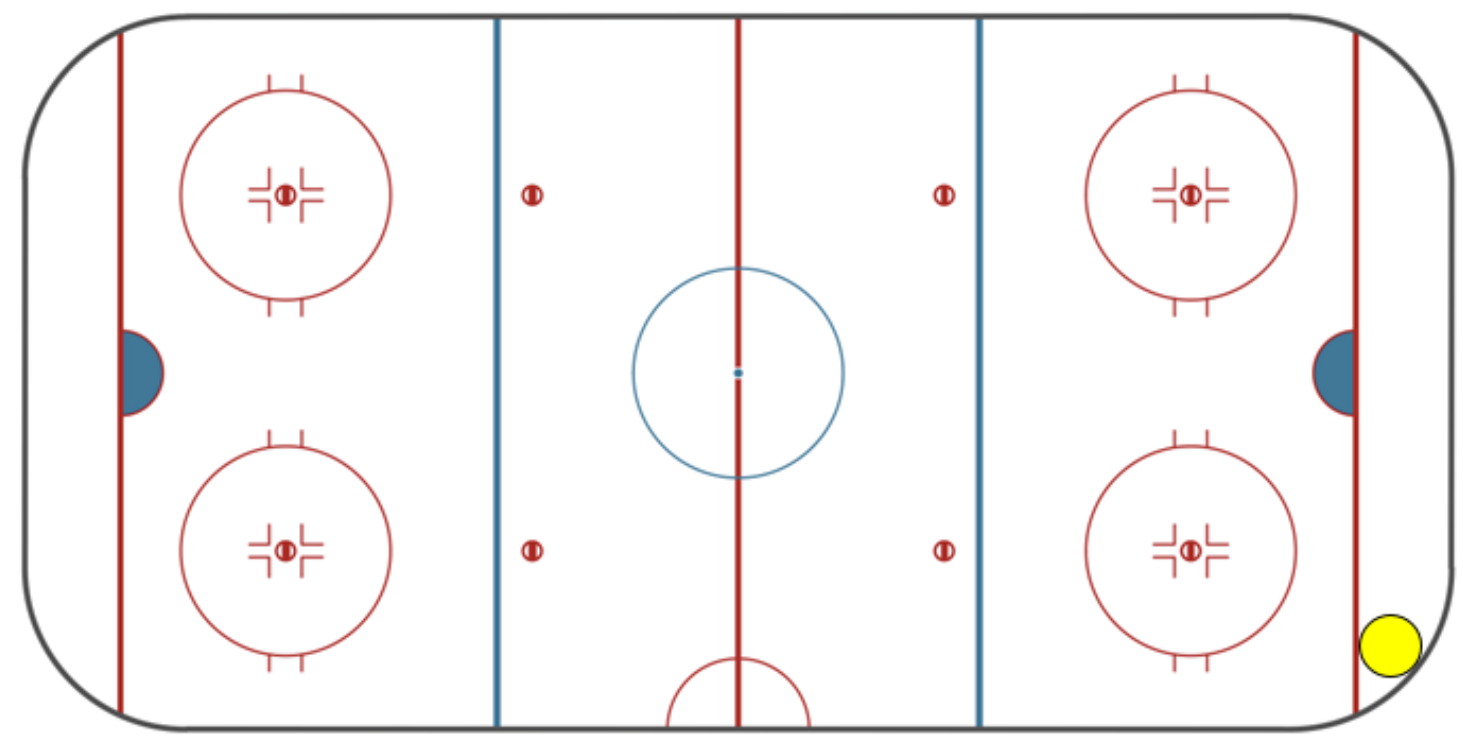

Figure 3.5: Scenario 2 
In Figure 3.5 the camera is placed by the boards in the corner. The scenario is made up of scenes 5-8.

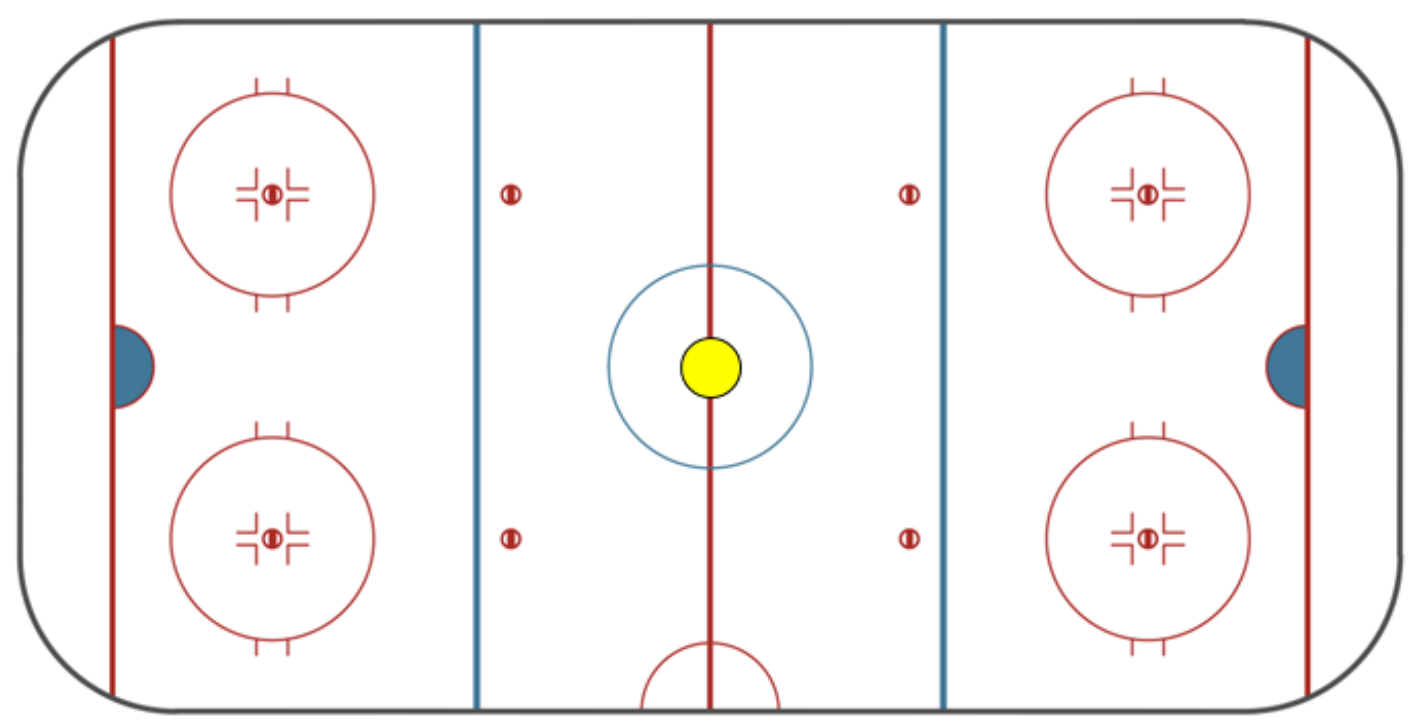

Figure 3.6: Scenarios 3 and 5

In Figure 3.6 the camera is placed in the center ice. Scenario 3 is made up of scenes 9-14 and provides the greatest challenge to the participants as it involves dual tasking (receiving the puck and reacting to a body-check) while also being spatially aware in all directions.

Scenario 5 is similar in that the camera is placed in the center ice however, there is no puck involved in the recordings. An opposing player will still skate towards the camera to simulate a body-check. This simplifies scenario 3 by removing the complexity of dual tasking, allowing the participant to focus on the one task of predicting which opposing players will body-check them. Scenario 5 is made up of scenes 19-22. 


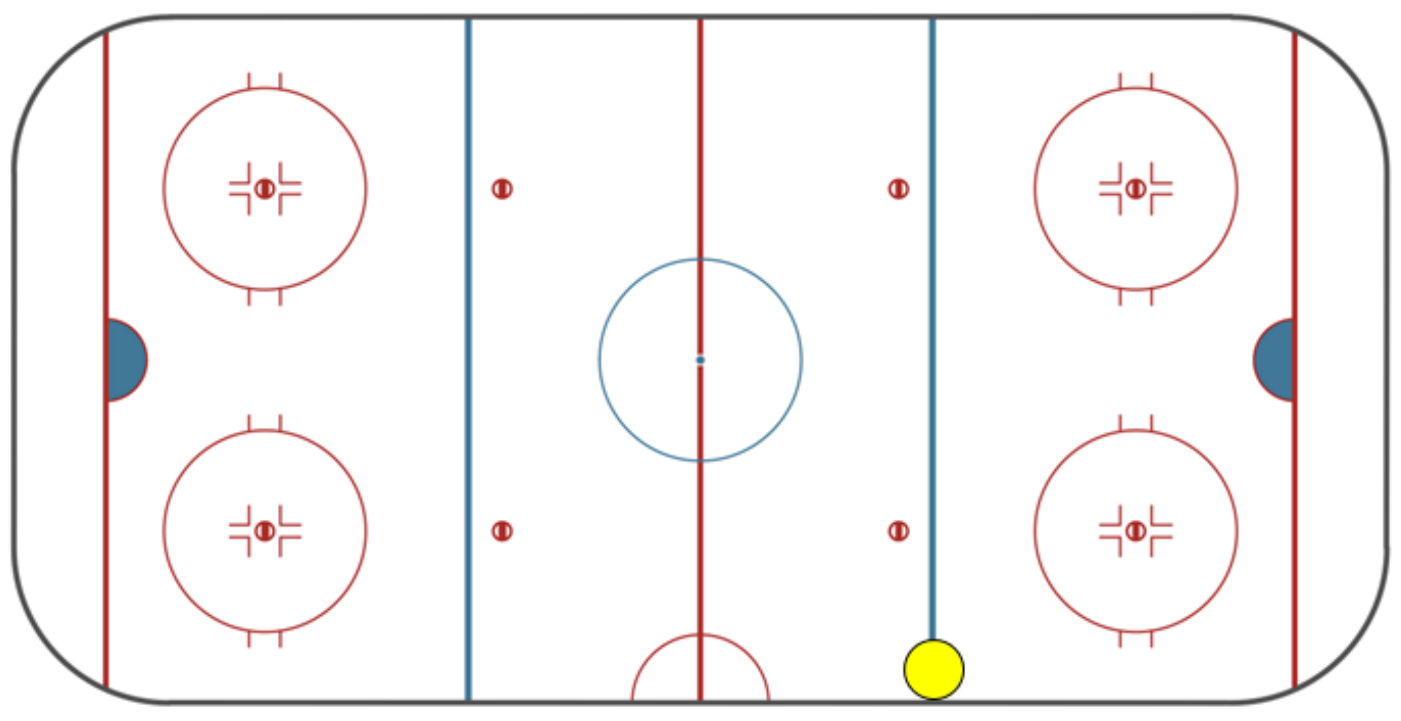

Figure 3.7: Scenario 4

Scenario 4 in Figure 3.7 is made up of scenes 15 -18 where the camera is placed on the blue line by the boards.

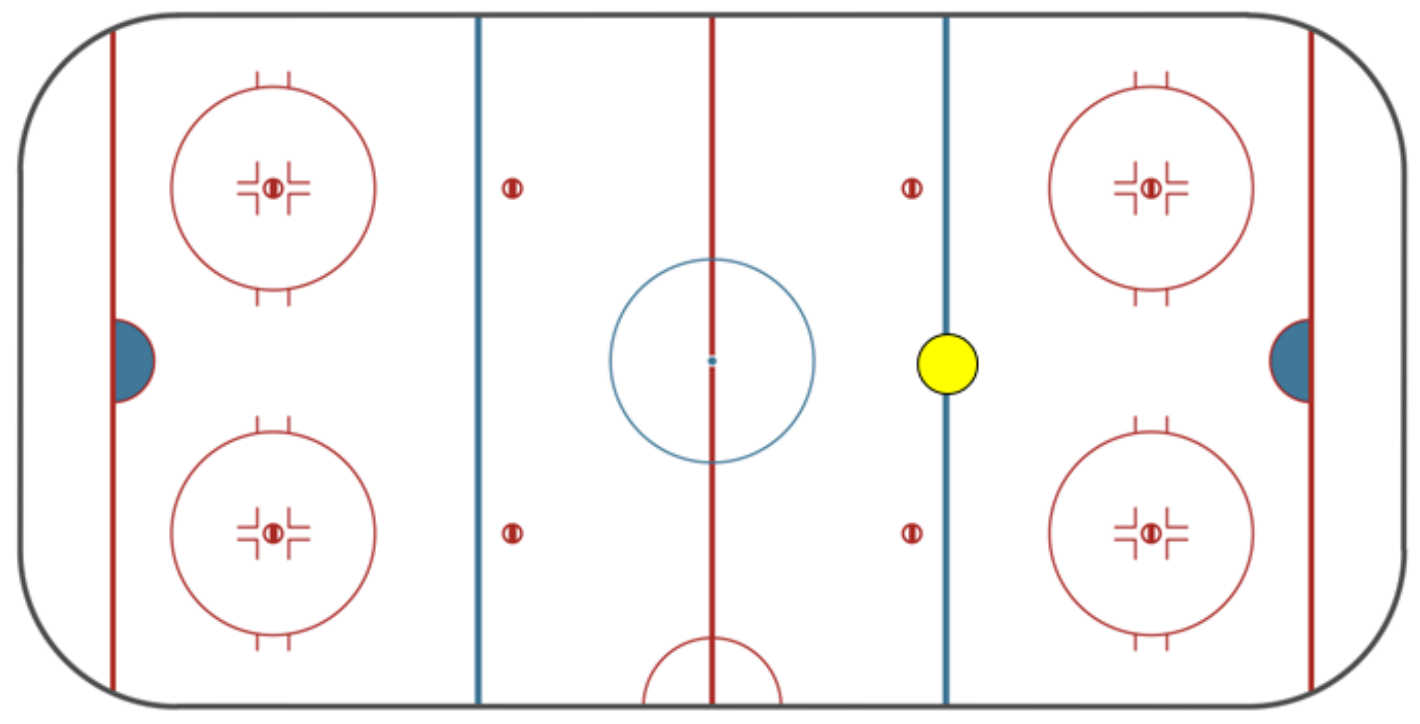

Figure 3.8: Scenarios 6 and 7 
In Figure 3.8 the camera is placed in the center of the blue line. Scenario 6 is made up of scenes 23-26 and scenario 7 is made up of scenes 27-30.

There are two distinct teams present in the recordings, one team wearing white jerseys and the second wearing black jerseys. In the simulation, one player wearing a white-jersey passes the puck towards the camera (i.e. the participant). Through all this, the black-jersey team is skating around the rink and as soon as the puck is passed towards the camera, a member from the black-jersey team charges towards the camera to simulate a body-check and the recording ends. Only 2 players wearing a black jersey are present in all 30 scenes. However, the number of players wearing white jerseys ranges from 2 to 4 .

Once all the scenarios have been recorded, both 360 video and audio were taken back to the lab for processing. In order to integrate the 360 videos with the Oculus Rift, Unity, a video game engine is programmed to playback videos to the participants.

Before actual testing, an introductory sample video is played to the participants in order to help familiarize them with the testing environment. The introductory video involves the puck being passed around in a circle with the participant in the middle observing. The participants are encouraged to look around in order to fully immerse in the virtual reality experience. The participants' are given as much time as required to become accustomed to the VR experience before the testing scenarios commence.

Figure 3.9 demonstrates a frame from the simulation of Scene 8 from Scenario 2. The participant is virtually by the boards in the corner of the rink looking towards the incoming puck as the opposing team-member is about to body-check the participant. 


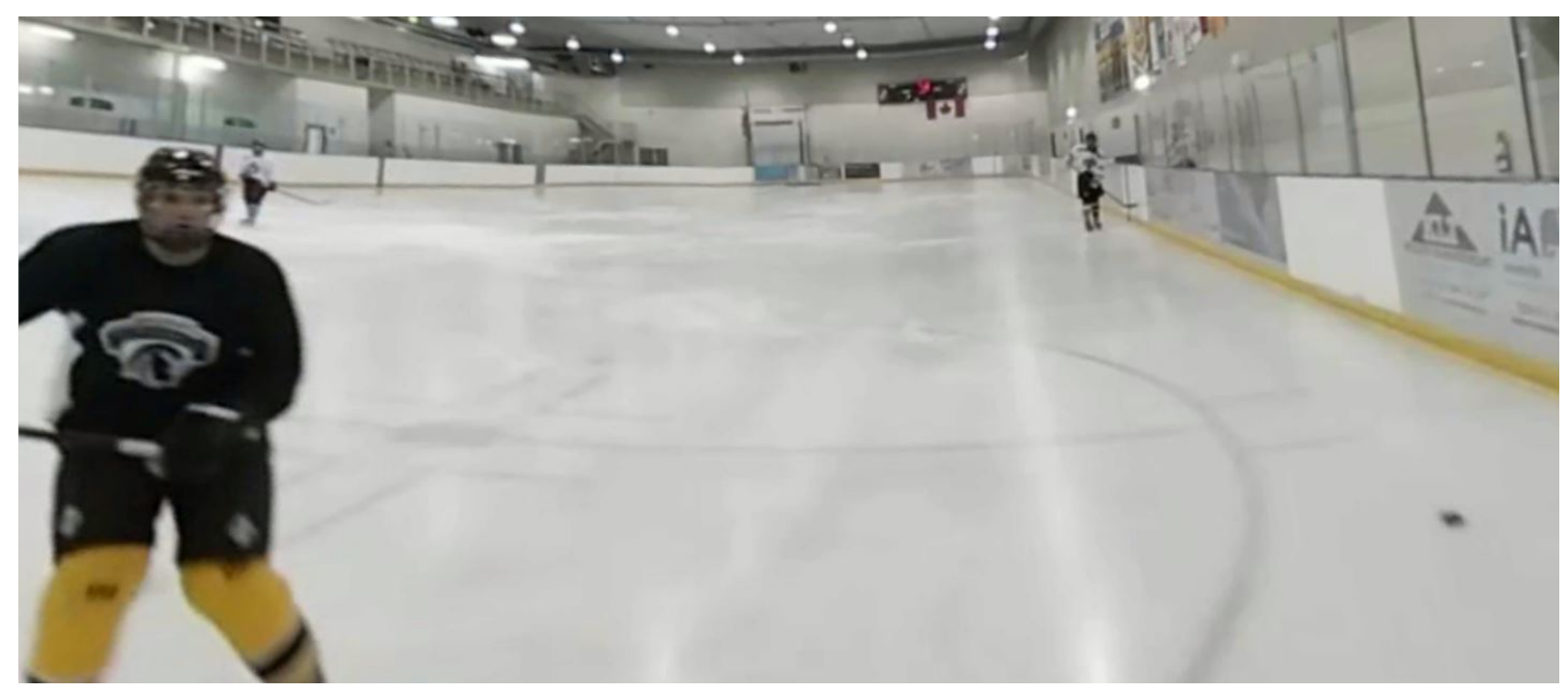

Figure 3.9: Real footage of Scene 8 from Scenario 2

Synthetic ice is placed on the floor to create a 9'x9' perimeter on which the participants can skate as shown in Figure 3.10. It is of utmost importance to create scenarios as realistic as possible such that the responses collected mimic the exact responses as would be seen in hockey games. Furthermore, a hockey stick is given to each participant to add to the realism of the experiment. 


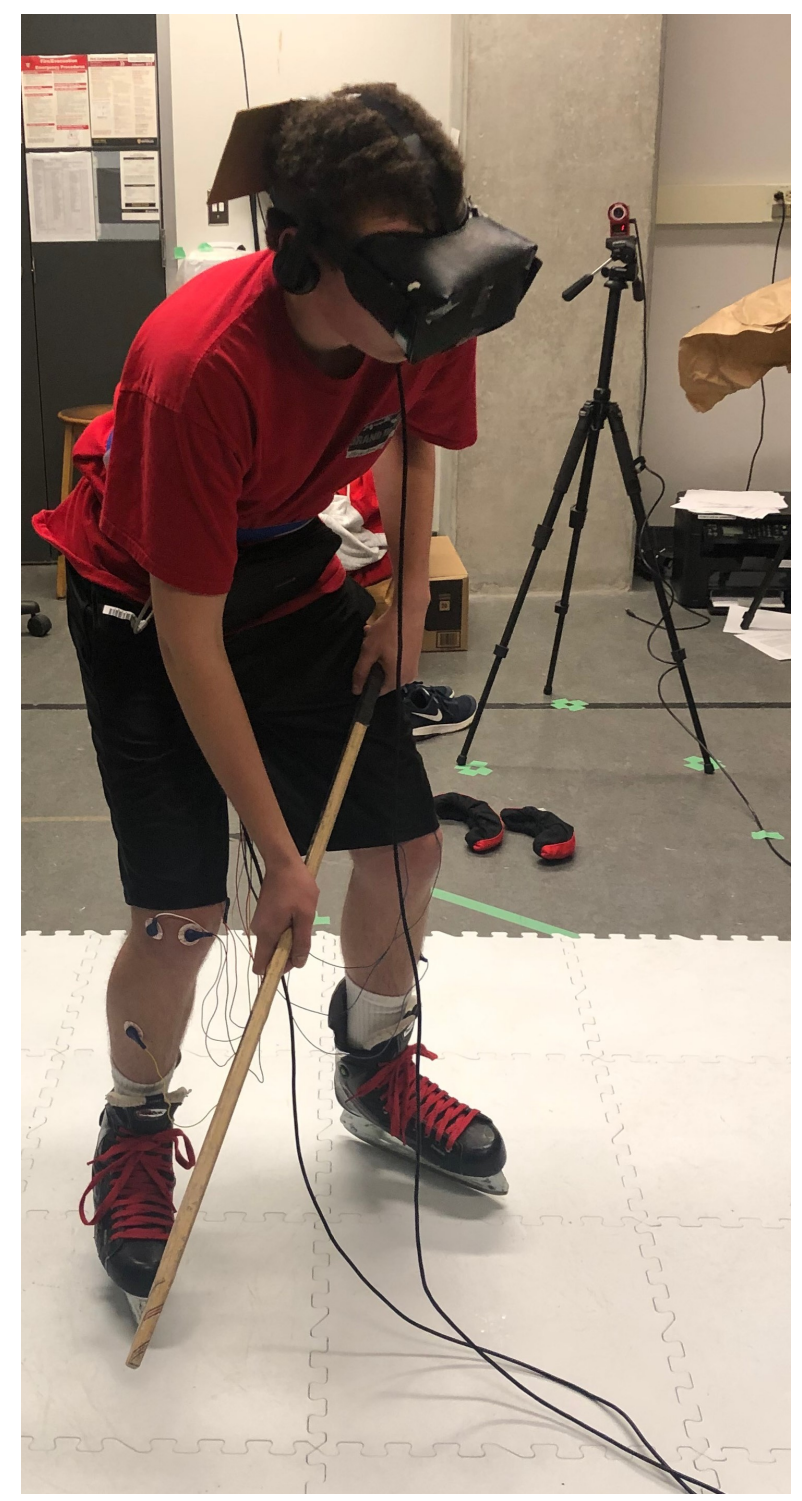

Figure 3.10: Participant with Skates on Synthetic Ice

Velcro-band is placed on the waist of the participants and on top of which 3 IR markers are placed as can be seen in Figure 1.1. The Velcro-band serves the purpose of minimizing any movement due to loose clothing. On the back of the Oculus Rift, 3 more IR markers are attached in order to monitor the motion of the head. Optitrack cameras placed in the testing laboratory capture 360 degree motion of the IR markers and serve the purpose of 
identifying both COM location and the directionality of the participant's head.

Figure 3.11 shows the real-time information of the IR markers during the experimental procedure. The 3 markers on the head are labeled as "Trackable 1" and the 3 markers on the waist are labelled as "Trackable 2". The participant is surrounded by 6 Optitrack cameras to ensure that each marker is captured by at least 2 cameras at every instance in time. The $\mathrm{X}-\mathrm{Z}$ plane marked on the figure is the plane that the participant skates on. The "Real-Time Information" tab on the right of the figure demonstrates the average values of the head markers "Trackable 1" during the experimental procedure. Of primary interest is the Yaw, the orientation around the Y-axis, which identifies where the participant is looking.

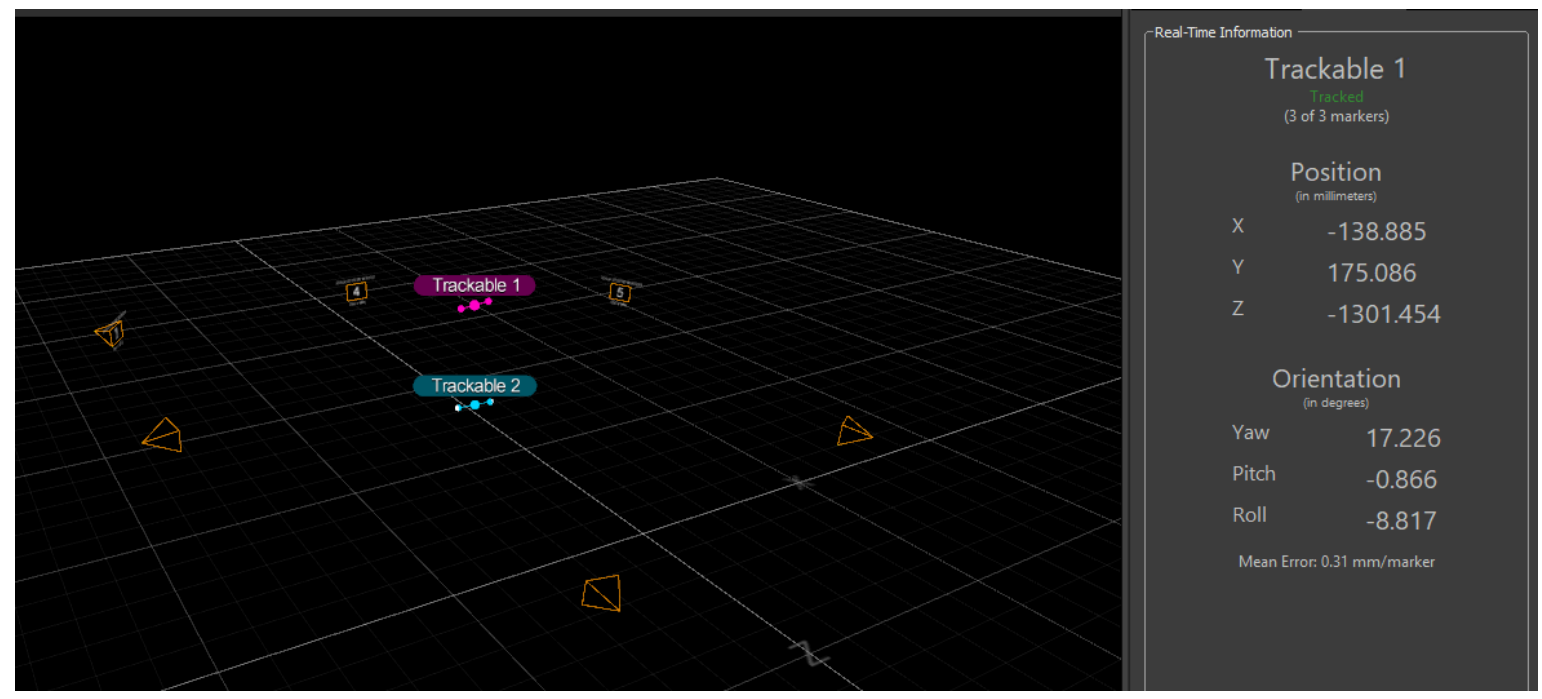

Figure 3.11: Real Time Visualization of Head Markers in Optitrack

When a testing scene is opened up, it is presented in a paused state to allow the participants to look around in all directions before the video is played. Once the participants are comfortable and ready, they inform the researchers. Parallel programming in the Unity game engine allows the researchers to start the video, start an Optitrack recording for the scene, and insert a trigger signal in the EMG data collection hub. The trigger signal allows for the comparison of the reaction times of different participants with respect to the start time of the scene. The trigger signal is also applied to Optitrack motion capture data so that the orientation of all participants can be compared per scene. Once the scene is complete, a researcher guides the participants to a default starting position at the beginning of every scene. This is beneficial as it helps avoid any wire entanglement and helps with the 
Optitrack data collection in that every scene is standardized with the participant facing the default position.

Figure 3.12 demonstrates the data flow direction between the different elements in the experimental procedure. There are 3 computer software applications running simultaneously. The Unity game engine application is responsible for streaming the different scenes through the Oculus Rift. Furthermore, as soon as a scene begins a trigger signal is sent to both the Optitrack cameras and an Arduino that relays the signal to the EMG hub. The Arduino is utilized to simplify the integration of Unity and the EMG hub. Optitrack cameras return the "Trackables" location and orientation to the Optitrack Tracking Tools software as was seen in Figure 3.11, this data is also stored in a text file. The EMG hub returns the measurements from the 8 electrodes to the "OpenBCI" software responsible for collecting and filtering EMG data.

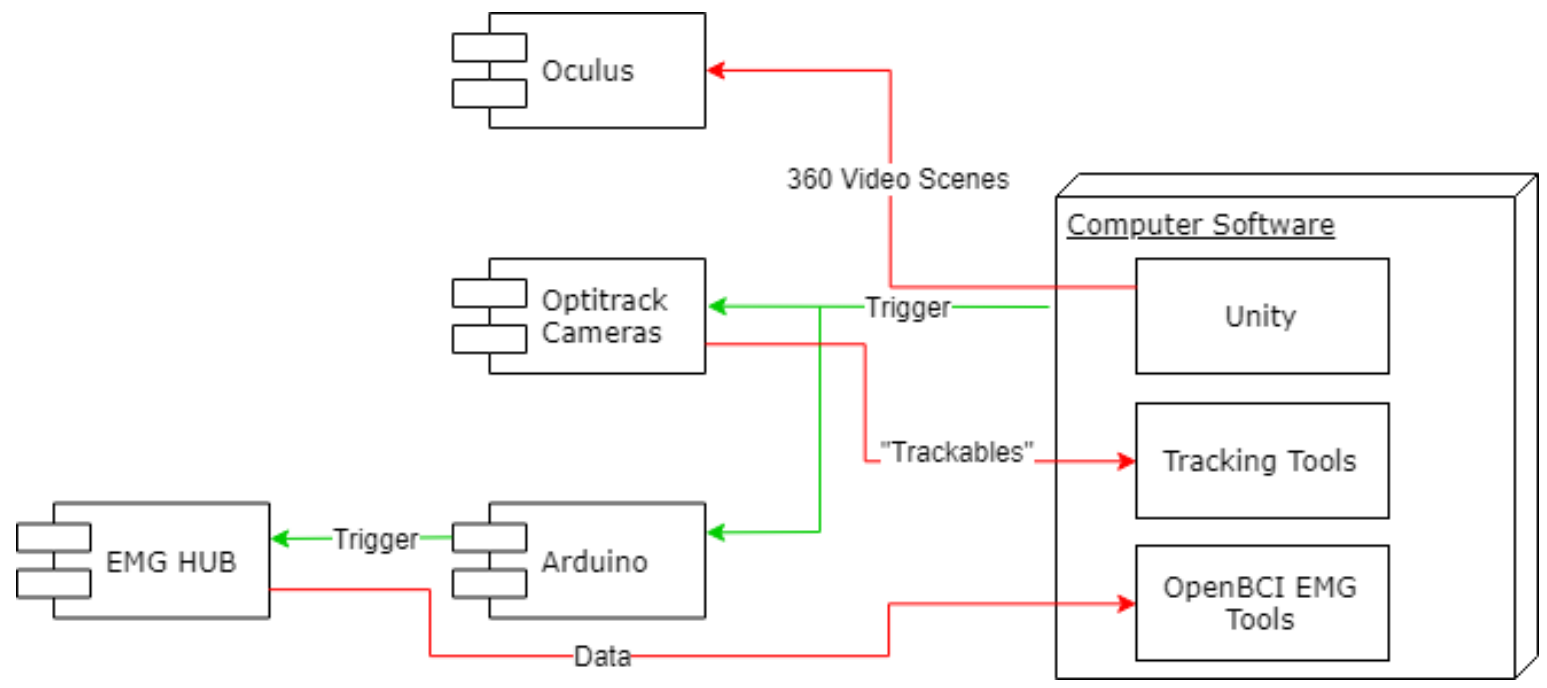

Figure 3.12: Communication between Different Components in the Experimental Procedure

Figure 3.13 shows the OpenBCI software application during EMG streaming. The data is streamed at $250 \mathrm{~Hz}$ and is automatically stored in a text file similar to Optitrack data. In all cases, live streaming from OpenBCI and Optitrack was monitored to ensure that there weren't any issues during the experimental procedure. 




Figure 3.13: OpenBCI EMG Data Streaming

This procedure is carried out until either all the scenarios have been completed, or the participant feels unwell to continue with the experiment, and at which point, the experimental procedure is discontinued. The pain scale shown in Figure 3.14 is displayed to participants in the case where the experiment is discontinued and therapists/physicians are required.

The pain scale was not used throughout the experimental procedure for any of the 13 participants, and there was no experience of discomfort that elicited the need of a physician or clinician. 


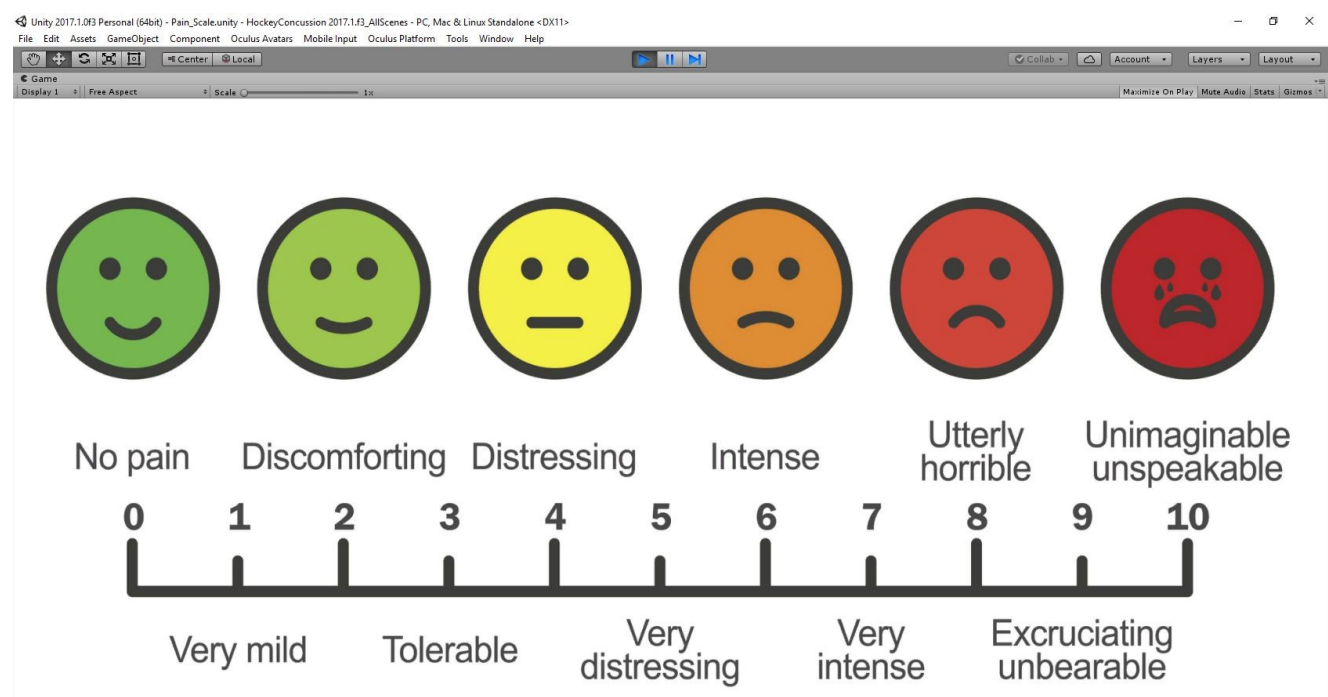

Figure 3.14: Pain Scale.

\subsection{Raw Data}

Upon completion of all 30 scenes for each participant, the Optitrack and EMG text files are imported into MATLAB for post processing and analysis.

Figure 3.15 presents the raw EMG data of the right rectus femoris of one participant for scene 2. Notice that there are four important markers superimposed on the graph. These represent the time at which the puck arrives to the player, the time at which the charging opponent reaches terminal velocity, the time at which collision occurs, and the time at which the scene ends. These four markers were extracted from each scene by playing back the videos in slow-motion at one-eighth the speed. The videos were paused when one of the events had occurred and the time was noted down. 


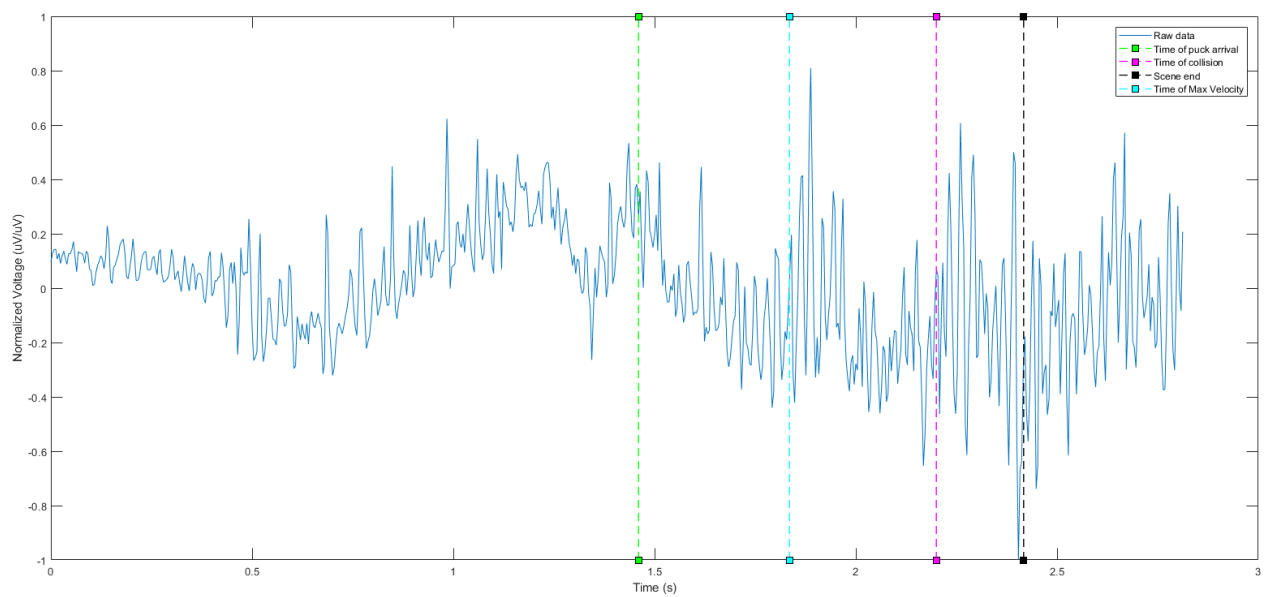

Figure 3.15: Raw EMG Data of the Right Rectus Femoris

Note that the y-axis in Figure 3.15 is normalized voltage. Voltage was normalized across all participants to create a standard metric for comparison. Let $x$ be a real-valued vector such that $x \in \mathbb{R}^{n}$ and $\mu$ the mean of $x$. The normalized voltage $\hat{x}$ is then:

$$
\hat{x}=\frac{x-\mu}{\max (|x-\mu|)}
$$

Figure 3.16 presents the directionality of a non-concussed participant during one scene. One of the observable differences between concussed and non-concussed participants is the statistical variance in the directionality. This observation could, with further research, support the fact that non-concussed participants are more spatially aware. Chapter 4 shows preliminary results for all participants during one scene. 


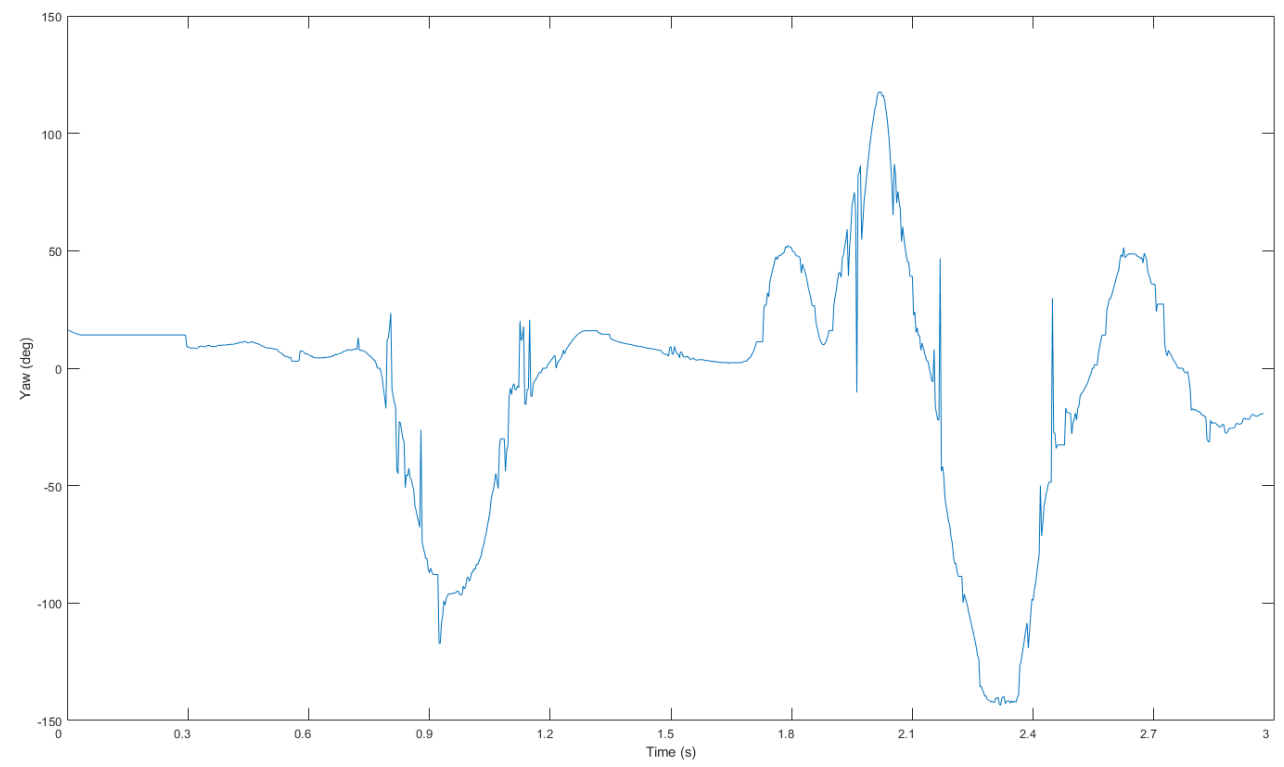

Figure 3.16: Head Directionality from a non-concussed Participant

Figure 3.17 presents the head directionality taken from a concussed participant for the same scene as used in Figure 3.16. It is clear that the maximum angle is smaller and that there aren't as many drastic rotations. This could possibly point to a lower spatial awareness. 


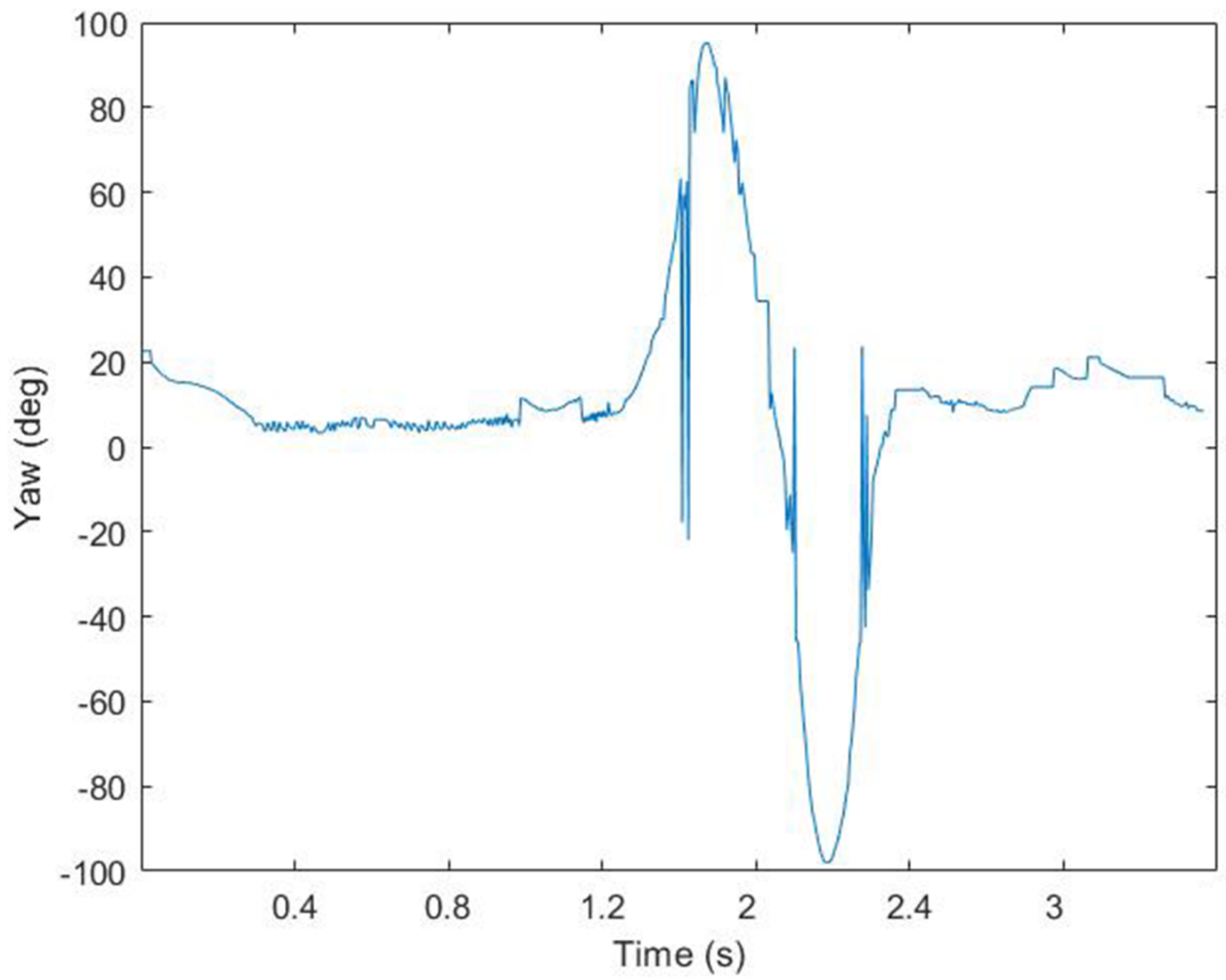

Figure 3.17: Head Directionality from a Concussed Participant

In the next chapter, EMG data will be post-processed and analyzed to display any significant differences between concussed and non-concussed hockey players. Due to limitations specified in Chapter 6, Optitrack data will be used qualitatively to support the notion that concussed participants are not as spatially aware as non-concussed participants. 


\section{Chapter 4}

\section{Results}

This chapter is concerned with reviewing signal analysis concepts needed for the processing of the captured signals.

\subsection{Signal Analysis Overview}

Given that the data captured by the EMG is noisy, it is necessary to apply signal processing to present the data embedded in the raw EMG signal in a manner which can be analyzed and interpreted by clinicians. This section goes through the basics of Fourier series, Fourier transforms, and finally wavelet transforms.

\subsubsection{Fourier Series}

A Fourier series breaks up an arbitrary periodic signal into an infinite sum of sines and cosines, by making use of the orthogonality relationship of the sine and cosine functions as well as Euler's formula.

The most general form of the Fourier series for a periodic real-valued function $f(t)$ can be written as follows [71]: 


$$
\begin{aligned}
f(t) & =\sum_{n=-\infty}^{\infty} A_{n} e^{i(2 \pi n t / L)} \\
A_{n} & =\frac{1}{L} \int_{-\frac{L}{2}}^{\frac{L}{2}} f(t) e^{-i(2 \pi n t / L)} d t
\end{aligned}
$$

Where $L$ is the period of $f(t)$.

\subsubsection{Fourier Transforms}

Fourier transforms are one of the most popular tools in wave decomposition and are used in representing aperiodic signals as an integral of sine and cosine functions. Used primarily for the purposes of decomposing a signal into its constituent frequencies, the transform has the following mathematical form [71]:

$$
X(\omega)=\int_{-\infty}^{\infty} x(t) e^{-j \omega t} \mathrm{~d} t
$$

Where $x(t)$ is the aperiodic time function, and $X(\omega)$ is the frequency domain representation of $x(t)$. The Fourier inversion theorem allows for the reconstruction of $x(t)$ from $X(\omega)$ as follows:

$$
x(t)=\frac{1}{2 \pi} \int_{-\infty}^{\infty} X(\omega) e^{j \omega t} \mathrm{~d} \omega
$$

However, for the purposes of this experiment, the Fourier transform has limitations. While localized in frequency, the transform not localized in time, and therefore is not as useful for this research.

\subsubsection{Short Time Fourier Transform Resolution Issues}

A STFT is similar to the Fourier transform seen in Equation (4.2) except that it determines the frequency contents of a signal as it changes over time. This is done by dividing a time signal into smaller segments of equal length and then computing the Fourier transform of 
each segment separately. This reveals the frequency content on each smaller time segment, and the changing spectra as a function of time can be plotted for illustration.

In order to calculate the STFT the time function of interest, $x(t)$ is multiplied by a window function $w(t)$ which is non-zero for a short period of time. The Fourier transform of the new signal is calculated as the window is translated across the time axis ( $\tau$ is the translation parameter). This is mathematically expressed as follows:

$$
X(\tau, \omega)=\int_{-\infty}^{\infty} x(t) w(t-\tau) e^{-j \omega t} \mathrm{~d} t
$$

In a STFT, there is a trade-off between time and frequency information determined by the width of the window function. A wide window in a STFT provides better frequency resolution than time resolution. This can be understood simply by extending the window width to approach infinity to emulate a Fourier transform. Conversely, a narrower width gives better time than frequency resolution. The example below demonstrates the difference in information when different window sizes are used for the Fourier Transform.

Consider the piecewise cosine function defined in Equation 4.5. There is one frequency present in four separate waveforms, $10 \mathrm{~Hz}, 25 \mathrm{~Hz}, 50 \mathrm{~Hz}$, and $100 \mathrm{~Hz}$ respectively.

$$
x(t)= \begin{cases}\cos (2 \pi 10 t) & 0 s \leq t<5 s \\ \cos (2 \pi 25 t) & 5 s \leq t<10 s \\ \cos (2 \pi 50 t) & 10 s \leq t<15 s \\ \cos (2 \pi 100 t) & 15 s \leq t<20 s\end{cases}
$$

The spectrograms in Figure 4.1 are produced with a sampling frequency of $400 \mathrm{~Hz}$. Four different subplots of different window sizes are shown.

In Figure 4.1a a $25 \mathrm{~ms}$ is the narrowest window that can be used since the original function is sampled at $400 \mathrm{~Hz}$ as stated before. The horizontal axis shows time in seconds, the vertical axis shows frequency in $\mathrm{Hz}$ and the colour-bar represents the magnitude of the frequencies. It is apparent that a narrow window covers time resolution well but frequency content is compromised. Observe that the predominant frequency changes at $5 \mathrm{~s}, 10 \mathrm{~s}$, and $15 \mathrm{~s}$. However the frequency content seems to have a range between $0-80 \mathrm{~Hz}$ from 0 to $5 \mathrm{~s}$, which is not true when referring back to Equation 4.5. Even if the largest amplitude is considered within the first 5 seconds by using the colour-bar, it still ranges from 0 to 30 $\mathrm{Hz}$, which is highly inaccurate in the case of EMG analysis. 
On the other hand, a $1000 \mathrm{~ms}$ window allows for greater frequency precision but worsens time precision between frequency changes as can be seen in Figure 4.1d. For example, it's quite clear that the predominant frequency in the first waveform is approximately $10 \mathrm{~Hz}$. However, the transition to the next frequency occurs around between $5-6 \mathrm{~s}$. It is clear from Equation (4.5) that a frequency change occurs at exactly $5 \mathrm{~s}$.

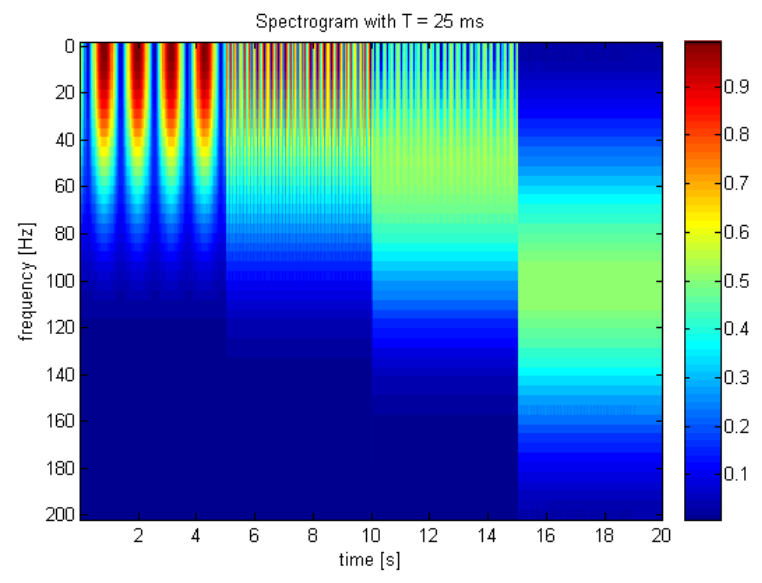

(a) A $25 \mathrm{~ms}$ window.

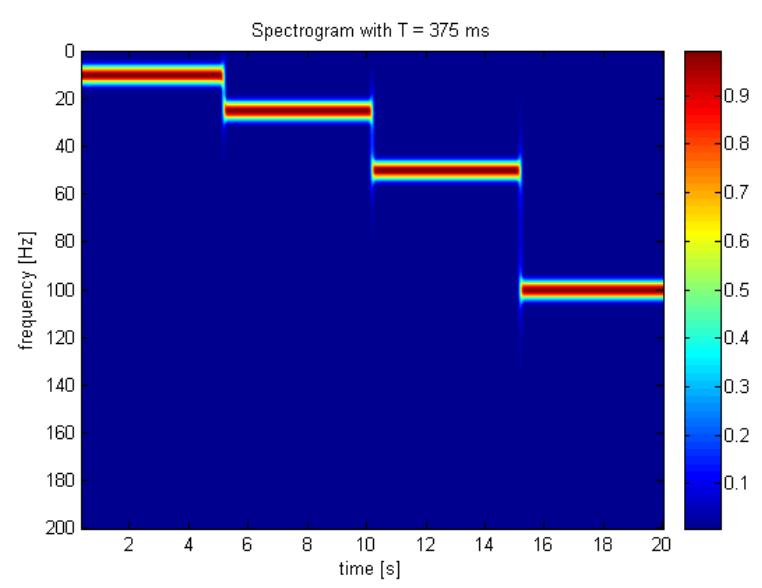

(c) A 375 ms window.

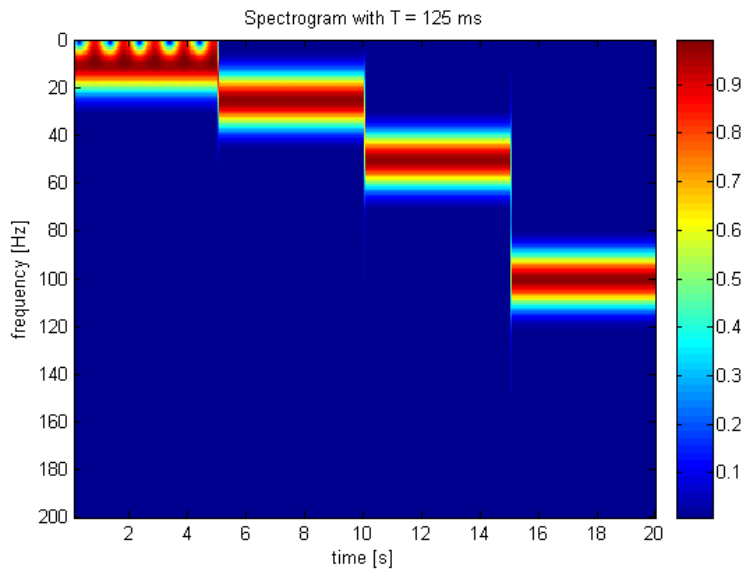

(b) A 125 ms window.

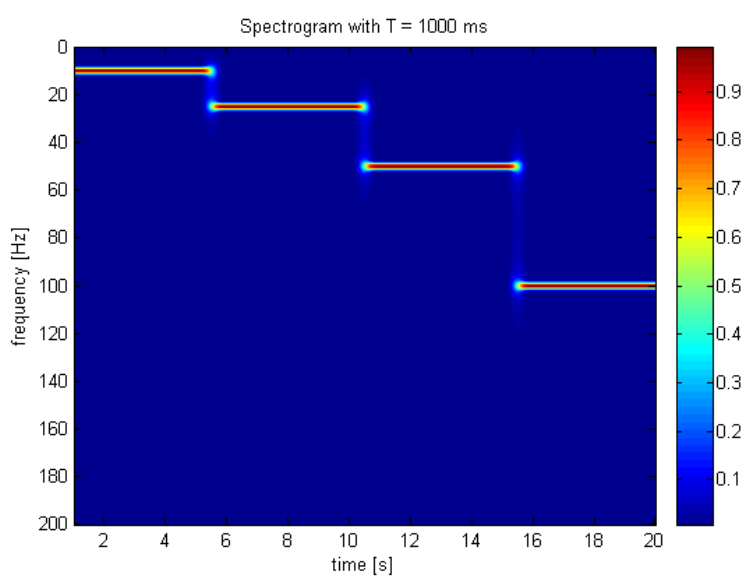

(d) A 1000 ms window.

Figure 4.1: STFT with different Window Sizes 


\subsubsection{Wavelet Transforms}

The Fourier transform is a useful tool in analyzing the frequency components of a signal; however, it is impossible to determine when a particular frequency arises. The STFT, in contrast, uses a sliding window to find a spectrogram which gives both time and frequency information. The problem with the STFT is that the width of the window function determines time and frequency resolution. Wavelet transforms provide a solution to this problem.

Wavelet transforms are based on wavelet functions with limited time duration and are governed by two important parameters, $j$ which represents the dilation of the wavelet and $k$ which represents the translation.

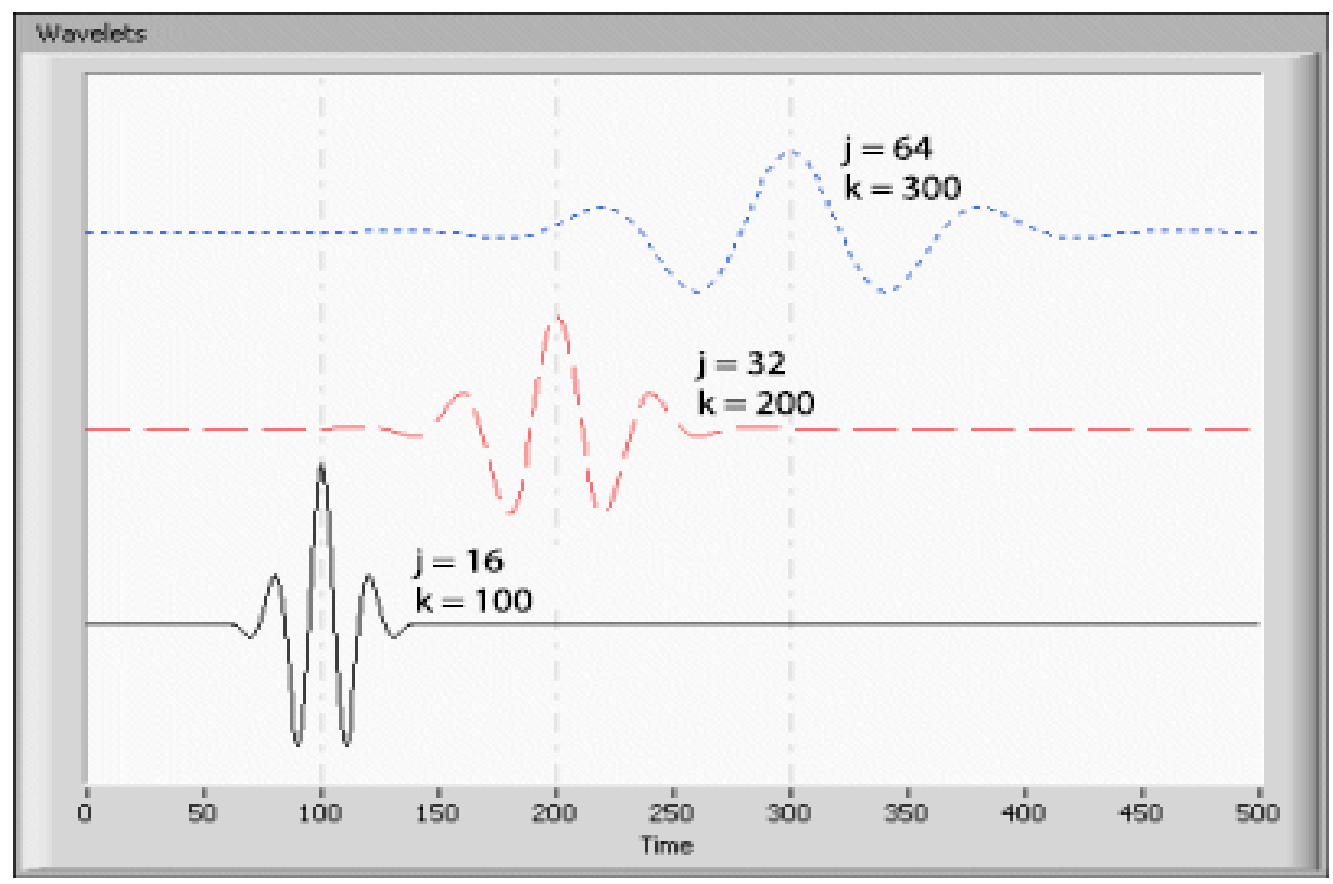

Figure 4.2: Morlet wavelets with different dilation and translation factors

Wavelet transforms are suitable for expressing both time and frequency information. This is ideal for displaying EMG data in that any clinician can identify muscle twitch activity for specific muscles throughout dynamic movement. It therefore gives a more illuminating method of expressing temporal changes in frequency. 
A function $\psi: \mathbb{R} \rightarrow \mathbb{R}$ is called a mother wavelet of order $m$ if the following five properties are satisfied [65]:

1. If $m>1$ then $\psi$ is $(m-1)$-times differentiable.

2. $\psi \in L^{\infty}(\mathbb{R})$. If $m>1$, for each $j \in\{1, \ldots, m-1\} \psi^{(j)} \in L^{\infty}(\mathbb{R})$.

3. $\psi$ and all its derivatives up to order $(m-1)$ decay rapidly: For each $r>0$ there is a $\gamma>0$ such that

$$
\left|\psi^{(j)}(t)\right|<\frac{1}{t}, \quad j \in\{0,1, \ldots, m-1\} \text { for each }|t|>\gamma .
$$

4. For each $j \in\{0,1, \ldots, m-1\}, \int t^{j} \psi(t) d t=0$. This is known as the vanishing moment property of the mother wavelet. A wavelet has $N$ vanishing moments $\Longleftrightarrow$ the wavelet scaling function (defined below) can generate polynomials up to degree $N-1$ and the wavelet coefficients are 0 for polynomials of degree at most $N-1$. The scaling function alone can be used to represent such functions.

5. The set $\left\{\psi_{j, k}\right\}_{j, k \in \mathbb{Z}}$ is an orthonormal basis of $L^{2}(\mathbb{R})$ where $\psi_{j, k}$ are derived from the mother wavelet by relationship $\psi_{j, k}(t)=2^{j / 2} \psi\left(2^{j} t-k\right)$.

Thus the expression for wavelet coefficients is given as

$$
f_{j, k}=\int_{-\infty}^{\infty} f(t) \psi_{j, k}(t) d t
$$

where $j$ and $k$ are related to frequency and time respectively.

Recall the example given by Equation (4.5) in Section (4.1.3) where the STFT was shown to be inaccurate for a time frequency analysis of a simple waveform. To overcome this, consider the implementation of the wavelet transform for Equation (4.5) in Figure 4.3 . 


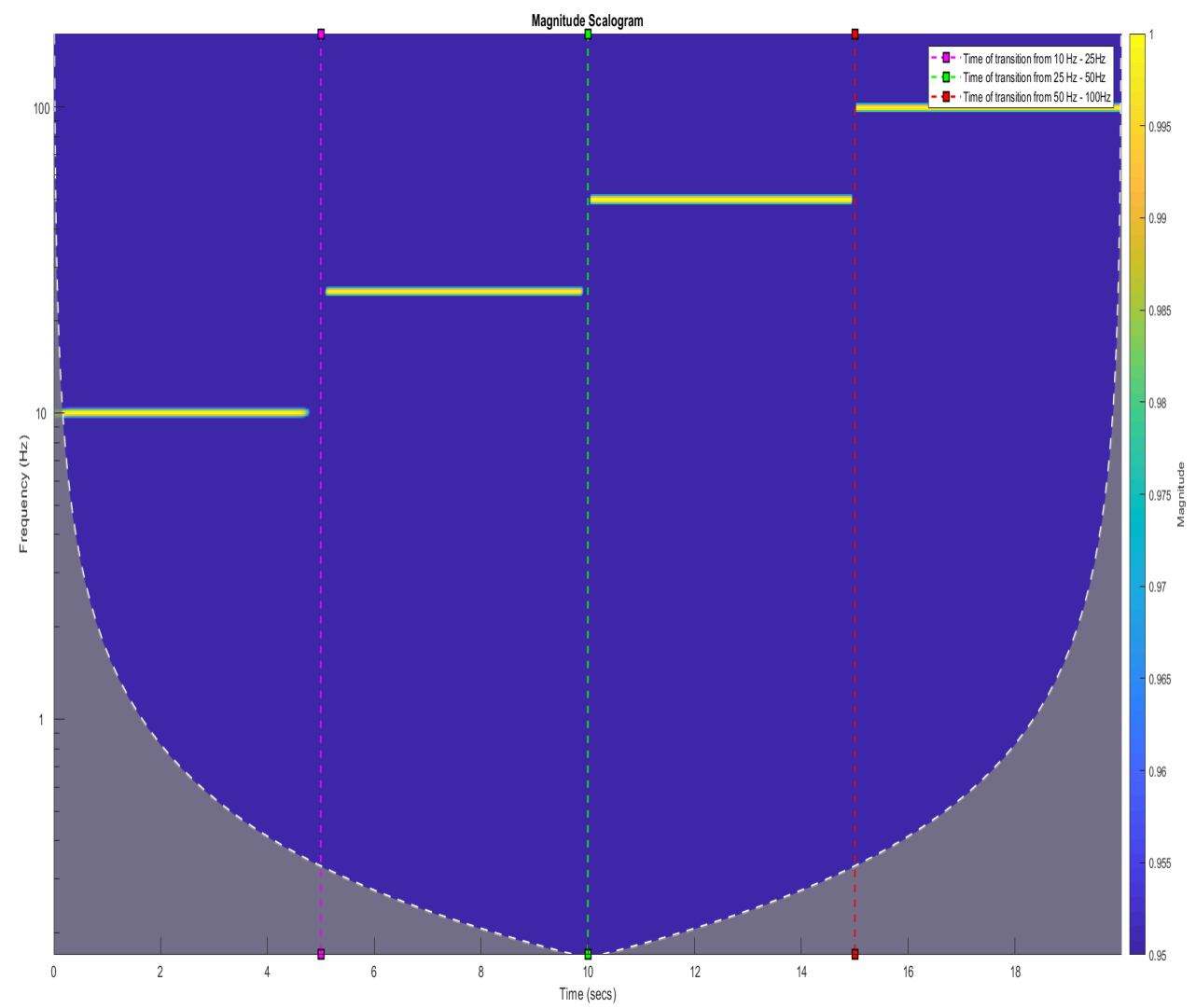

Figure 4.3: Wavelet Transform of Equation 4.5

Figure 4.3 shows the time frequency plot for Equation (4.5). The $x$ - axis represents time and the $y$ - axis represents frequency. The colour-bar shows the magnitude of the frequencies based on the wavelet coefficients. Three markers are superimposed on the graph to depict when a frequency change should occur. The dashed white line and the grey region represent the cone of influence. The cone of influence shows areas in the scalogram potentially affected by edge-effect artifacts. These are effects in the scalogram that arise from areas where the stretched wavelets extend beyond the edges of the observation interval. Values in the grey region should therefore be treated with caution as they are susceptible to edge-effects. [68]. 


\subsection{EMG Post-Processing}

EMG data was collected from 13 different participants, 6 who had suffered at least 1 concussion in the past. Post-processing was done on all 6 muscles from the 13 participants for the 30 different scenes.

The strategy for post-processing EMG data is outlined in Figure 4.4. The raw EMG signal is denoised using a Daubechies Discrete Wavelet Transform (DDWT). A CWT is then applied to the denoised signal to plot the time-frequency behaviour of the signal for illustrative and visual purposes. PCA is then applied to the denoised EMG signal in order to identify any differences in the reaction times of concussed and non-concussed hockey players. Once a visual intuition was built up from the PCA plot, Box plots were utilized to output the means and variances of the first two critical components for the different groups of interest.

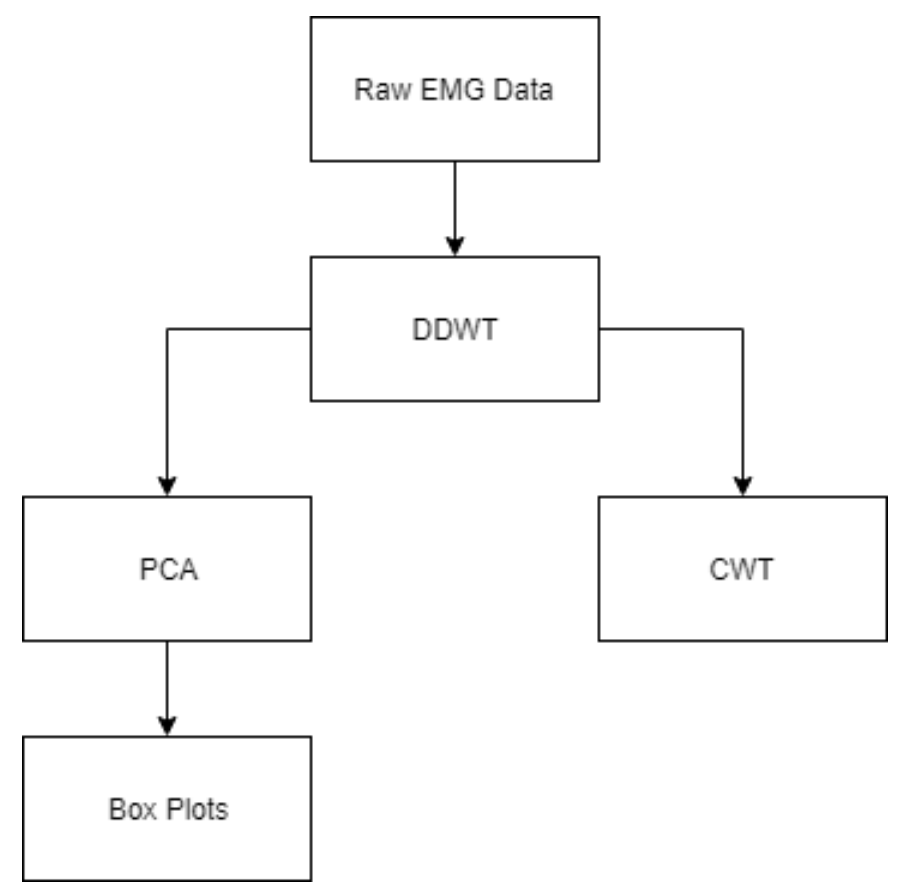

Figure 4.4: Post-processing Technique 


\subsubsection{Discrete Wavelet Transform}

In the case of EMG analysis, the Daubechies wavelets are the most widely used wavelet functions for EMG feature extraction. Daubechies wavelets also act as bandpass filters and are capable of effectively removing more unnecessary noise in the EMG signal when compared to Butterworth filtering techniques [56]. Mallat has introduced a repetitive application of high pass and low pass filters to calculate the wavelet expansion of a given function as shown in Figure 4.5 [41, 60].

An auxiliary function $\phi(t)$, a companion of the wavelet function, which is called the scaling function, is used to define the approximations (low-pass output) and forms a set of orthonormal bases of $L^{2}(\mathbb{R})$ as given by [11]:

$$
\phi_{j, k}(t)=2^{-j / 2} \phi\left(\frac{t-k 2^{j}}{2^{j}}\right), \quad j, k \in \mathbb{Z} .
$$

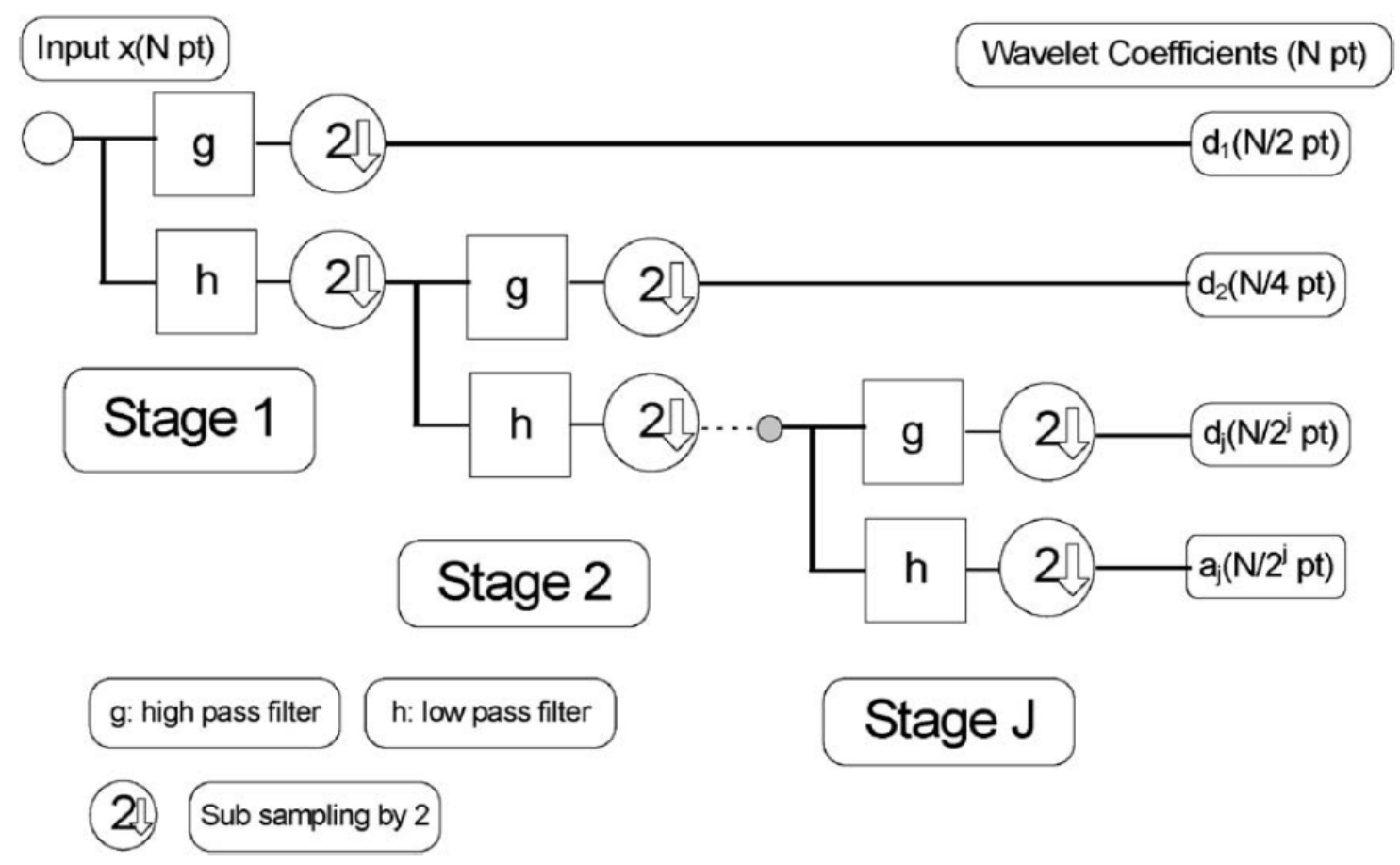

Figure 4.5: Mallat's cascaded filter [65] 
The scaling function $\phi(t)$ satisfies

$$
\int_{-\infty}^{+\infty} \phi(t) d t=1
$$

and two scale difference equation,

$$
\phi(t)=\sqrt{2} \sum_{k=0}^{L-1} h_{k} \phi(2 t-k), \quad j, k \in \mathbb{Z} .
$$

The wavelet function is given by

$$
\psi j, k(t)=2^{-j / 2} \psi\left(\frac{t-k 2^{j}}{2^{j}}\right), \quad j, k \in \mathbb{Z} .
$$

In Equation (4.10), the function $\psi$ has $M$ vanishing moments up to order $(m-1)$ and it satisfies the following two scale difference equation,

$$
\psi(t)=\sqrt{2} \sum_{k=0}^{L-1} g_{k} \phi(2 t-k) .
$$

Recall that the wavelet transform requires a pair of filters (high-pass and low-pass). One filter in the pair calculates wavelet coefficients (high-pass), whereas, the other applies the scaling function (low-pass). This scaling function, implemented with filter coefficients $\left\{h_{k}\right\}$, provides an approximation of the signal via the following equation:

$$
W_{L}(n, j)=\sum_{m} W_{L}(m, j-1) h(m-2 n) .
$$

The wavelet function gives us the detail signals, which are also called high-pass coefficients, and they are given by the following equation:

$$
W_{H}(n, j)=\sum_{m} W_{L}(m, j-1) g(m-2 n),
$$

where $W_{L}(p, q)$ is the $p$ th scaling coefficient at the $q$ th stage, $W_{H}(p, q)$ is the $p$ th wavelet coefficient at the $q$ th stage, and $h(n), g(n)$ are the filter coefficients corresponding to the 
scaling (low-pass filter) and wavelet (high-pass filter) functions respectively. These two filters are called quadrature mirror filters and are related by

$$
g_{k}=(-1)^{k} h_{L-K}, \quad k=0, \ldots, L-1 .
$$

It is common practice to use a $7^{\text {th }}$ order Daubechies wavelet (db7) to iteratively transform the EMG signal into subsets of coefficients [56]. The db7 wavelet and scaling functions are presented in Figure 4.6.

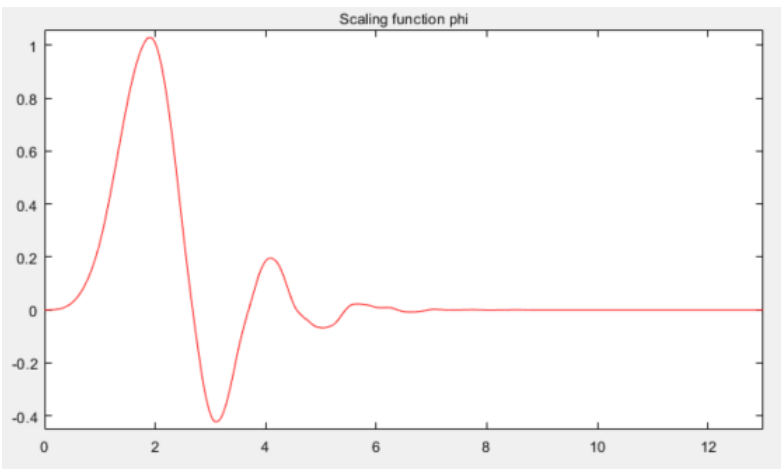

(a) db7 Scaling Function $\phi$

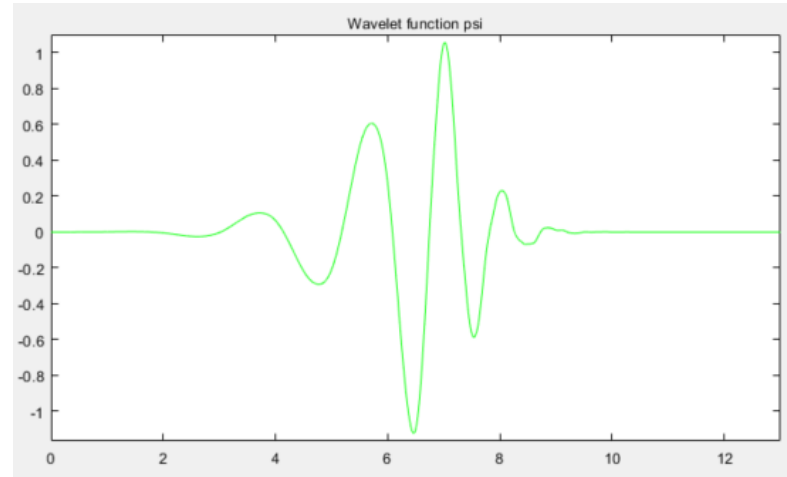

(b) db7 Wavelet Function $\psi$

Figure 4.6: Daubechies 7th Order Wavelet 


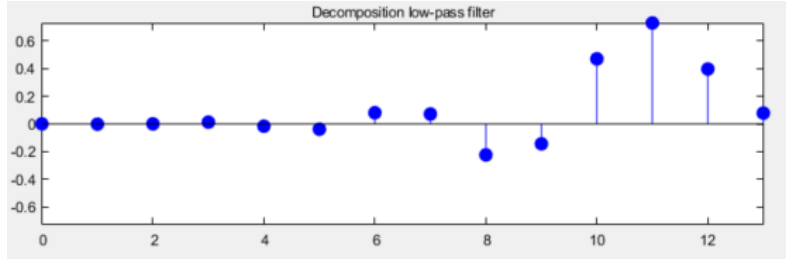

(a) $\phi$ Decomposition low-pass filter

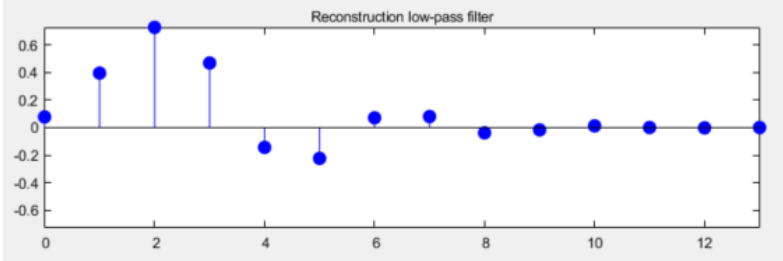

(c) $\phi$ Reconstruction low-pass filter

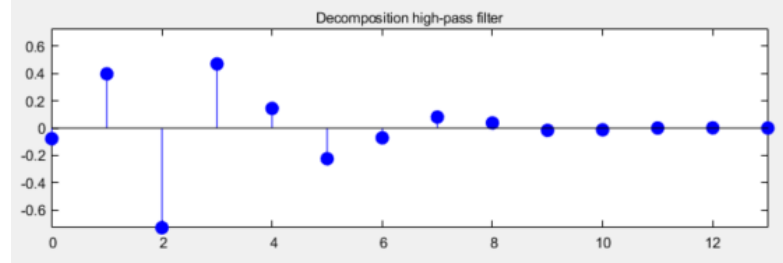

(b) $\psi$ Decomposition high-pass filter

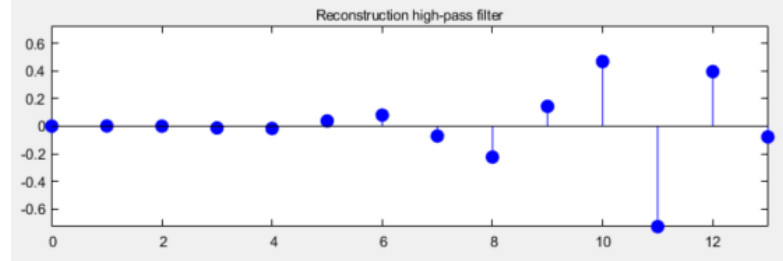

(d) $\psi$ Reconstruction high-pass filter

Figure 4.7: db7 Decomposition and Reconstruction of the Scaling and Wavelet Functions

It has been shown previously that Detail levels ' $\mathrm{d} 1$ ' and ' $\mathrm{d} 2$ ' are sufficient in encompassing all the important details in a raw EMG signal [24, 55, 56].

Consider the decomposition of a raw EMG signal into 4 levels using the Daubechies Discrete Wavelet Transform (DWT) shown in Figure 4.8 


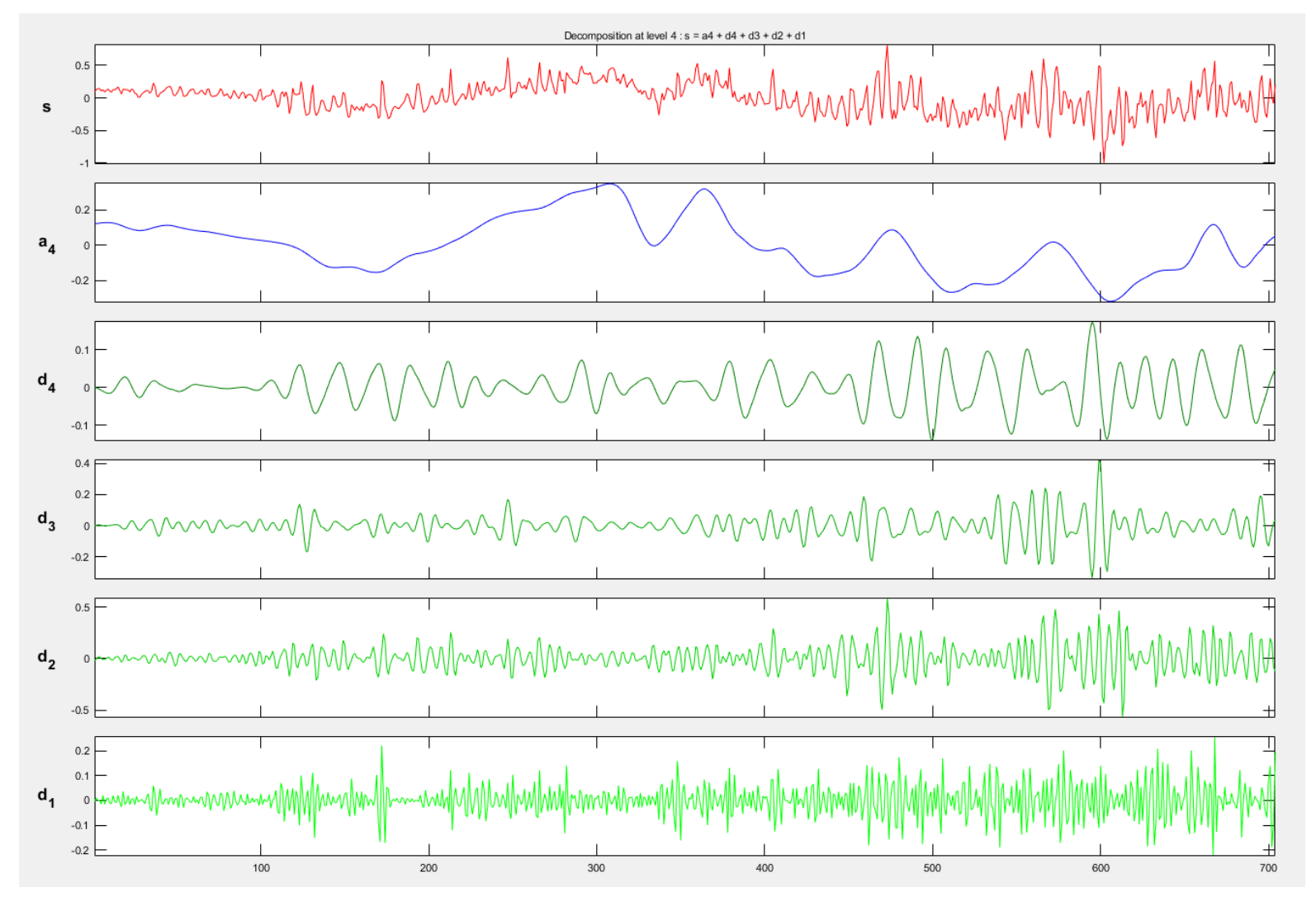

Figure 4.8: EMG Decomposition

The reconstructed EMG waveform using detail levels 1 and 2 is shown in Figure 4.9. The raw signal is shown in red, and the superimposed denoised signal is shown in black. 


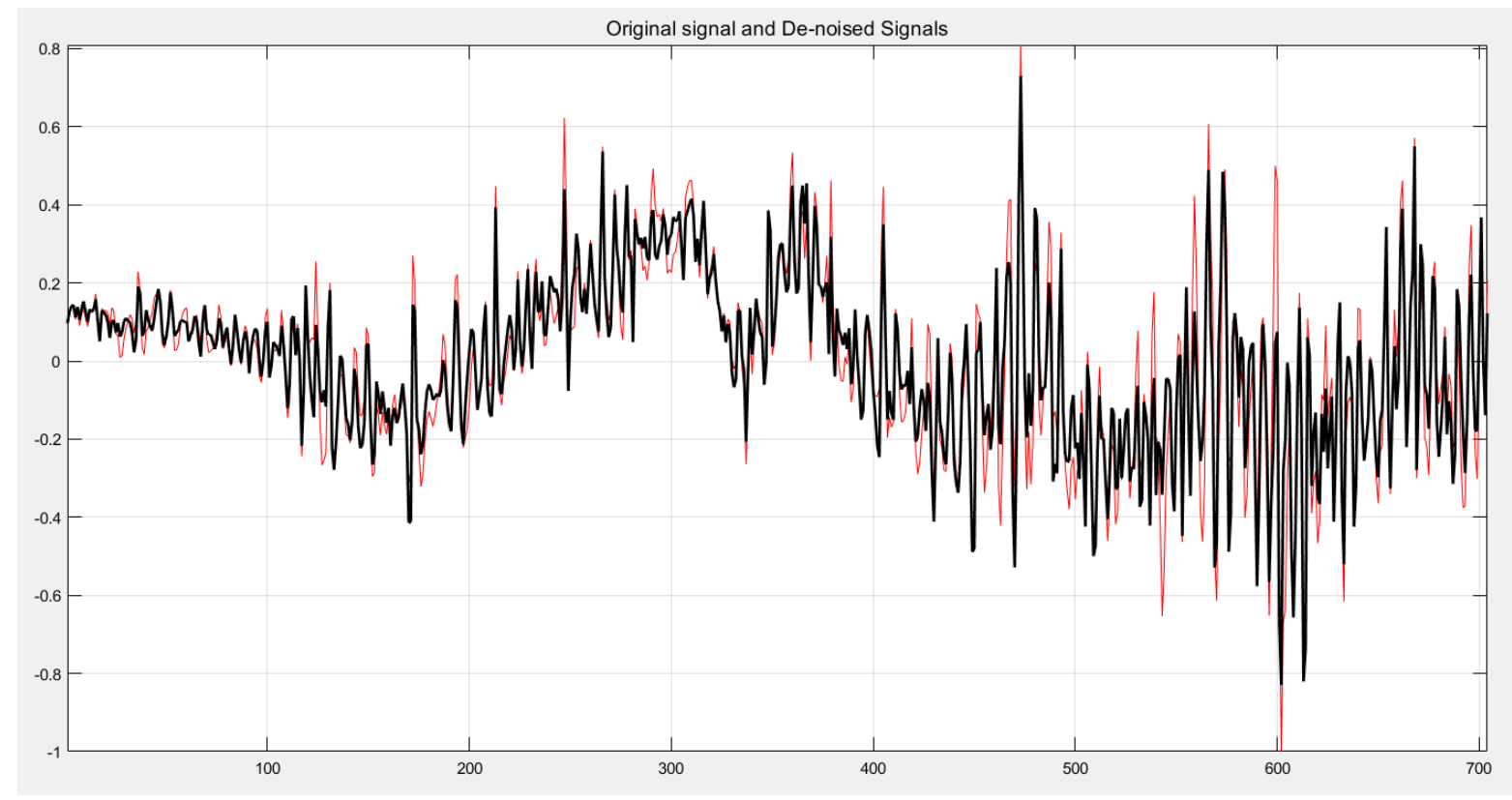

Figure 4.9: Denoised EMG Signal in Black, Original in Red

Once the EMG data has been denoised, the maximum EMG peak that occurs between the terminal velocity of the opposing player and the time of collision identified. The peak time is then subtracted from the time of the terminal velocity to yield reaction time.

The reaction time in Figure 4.10 as an example would be the time of the peak response $(t=1.888)$ minus the time of terminal velocity $(t=1.835)$. 


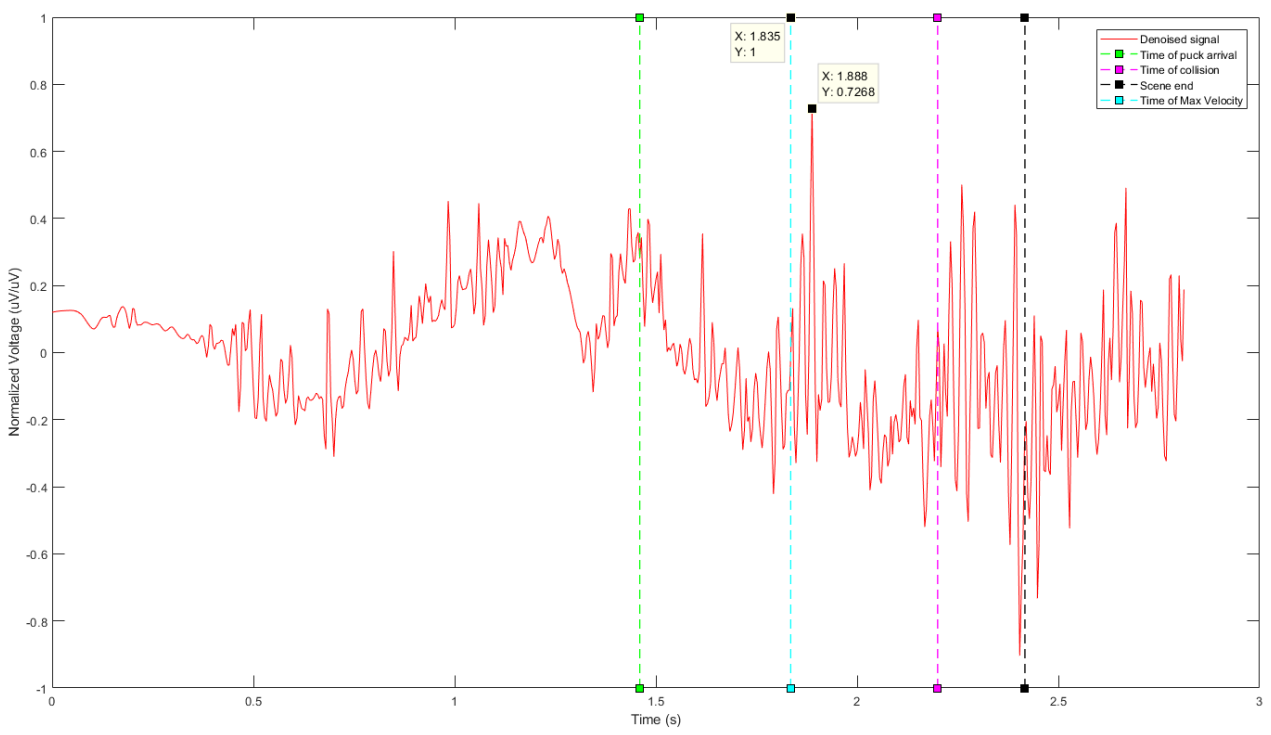

Figure 4.10: Reaction Time Visualization

\subsubsection{Continuous Wavelet Transforms}

There are several types of wavelets that can be utilized depending on the application and the signal at hand. However, the most general expression for a mother wavelet can be written as:

$$
\Psi_{a, b}(t)=\frac{1}{\sqrt{a}} \psi\left(\frac{t-b}{a}\right)
$$

where $a, b \in \mathbb{R}$ and $a \neq 0$. $a$ is the scaling factor that determines the window size and $b$ is the translation factor. Recall that for a STFT $a$ was fixed. This is not the case with wavelets.

The wavelet transform is defined in Equation (4.16) as the integral of the mother wavelet with the time function of interest $x(t)$ :

$$
X(a, b)=\frac{1}{\sqrt{a}} \int_{-\infty}^{\infty} \psi\left(\frac{t-b}{a}\right) x(t) \mathrm{d} t
$$


where, $\psi(\cdot)$ represents the desired mother wavelet, and $a, b$ represent scaling and time variables respectively. The choice of the mother wavelet depends on the intended application and range of frequencies of interest. For the purposes of EMG analysis, the Morse wavelet was used to analyze the time frequency components as proposed by Wachowiak et al. [70].

The Fourier transform of the generalized Morse Wavelet is

$$
\Psi_{P, \gamma}(\omega)=U(\omega) a_{P, \gamma} \omega^{\frac{P^{2}}{\gamma}} e^{-\omega^{\gamma}}
$$

where $U(\omega)$ is the unit step, $a_{P, \gamma}$ is a normalizing constant, $P^{2}$ is the time-bandwidth product, and $\gamma$ characterizes the symmetry of the Morse wavelet. By adjusting the timebandwidth product and symmetry parameters of a Morse wavelet, analytic wavelets with different properties and behavior emerge.

The plots in Figure 4.11 show how different values of symmetry and time-bandwidth affect the shape of a Morse wavelet. Longer time-bandwidths broaden the central portion of the wavelet and increase the rate of the long time decay. Increasing the symmetry broadens the wavelet envelope, but does not affect the long time decay. Very small time-bandwidth and large symmetry values produce undesired time-domain sidelobes and frequency-domain asymmetry $[36-38,50]$. The $P^{2}$ and $\gamma$ values used are based off the work of Wachowiak et al. [70]. 


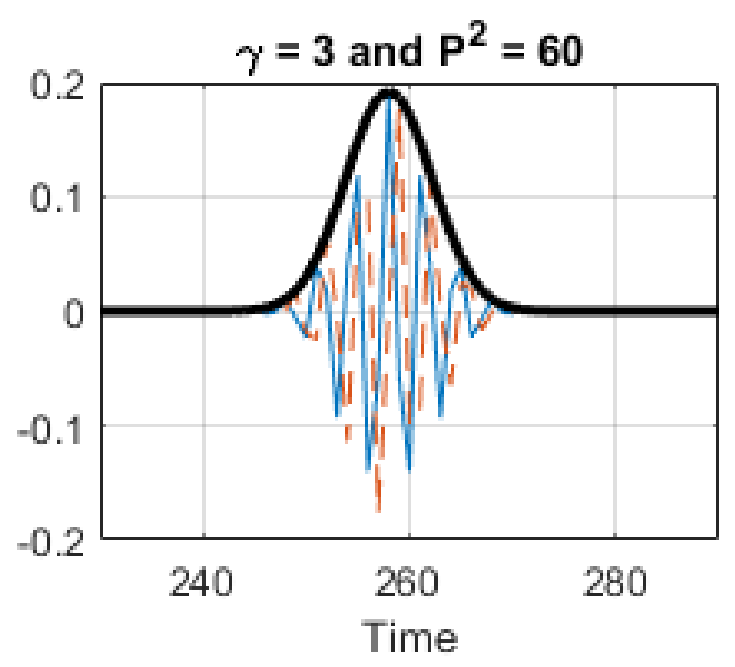

(a) $\gamma=3$ and $P^{2}=60$

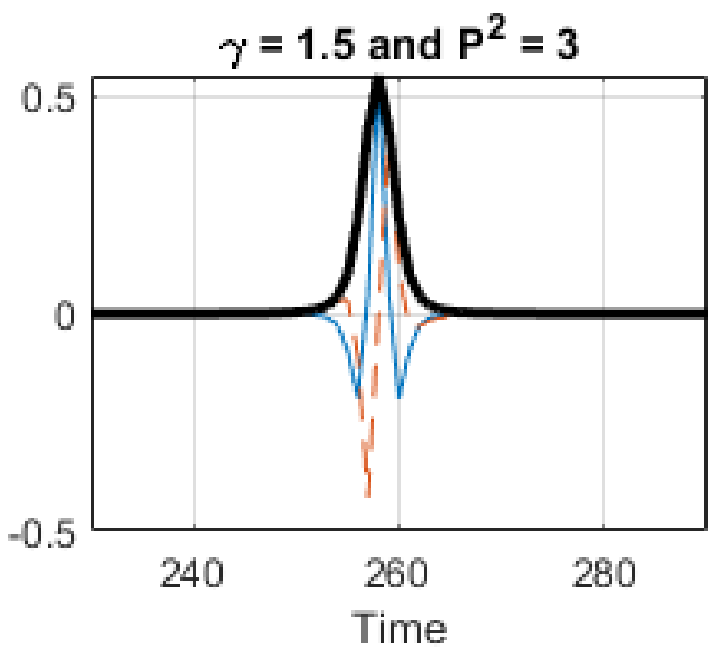

(c) $\gamma=1.5$ and $P^{2}=3$

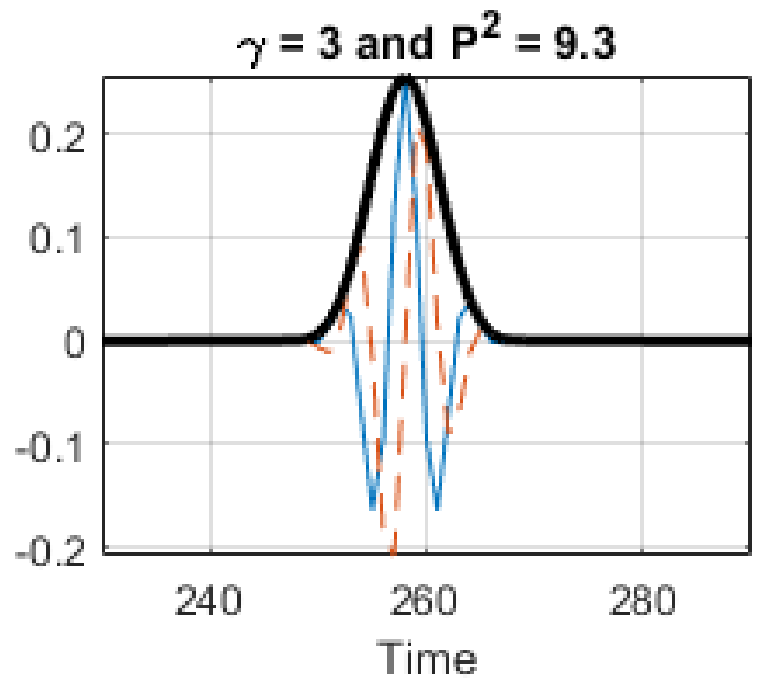

(b) $\gamma=3$ and $P^{2}=9.3$

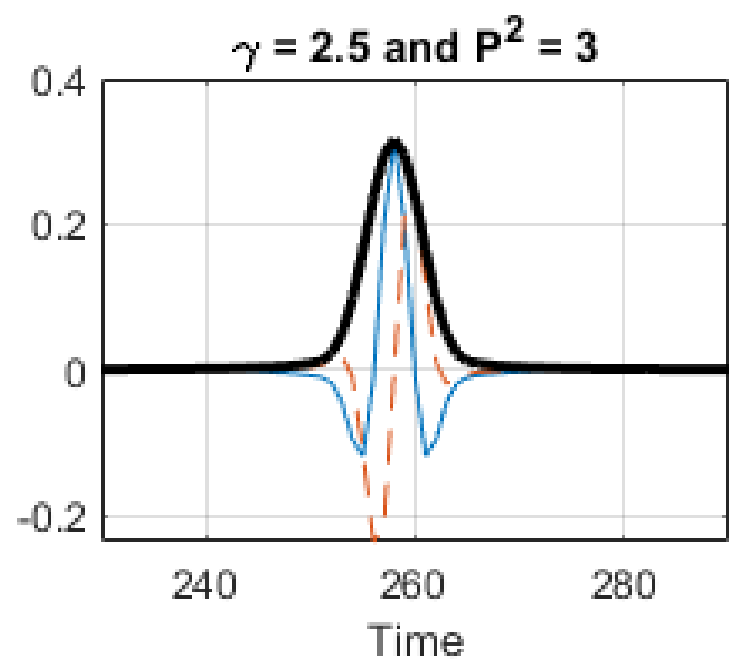

(d) $\gamma=2.5$ and $P^{2}=3$

Figure 4.11: Effects of symmetry and time-bandwidth values on the shape a Morse wavelet

Figure 4.12 shows the time frequency plot of the denoised EMG signal from Figure 4.9. The plot is the response of the right rectus femoris of a non-concussed participant for scene 2. Note the clear response of the rectus femoris muscle around $1.8 \mathrm{~s}$, right after the 
opposing player reaches terminal velocity (shown by the cyan marker). This non-concussed participant reacts almost instantaneously with more than $500 \mathrm{~ms}$ before collision occurs (shown by the magenta marker).

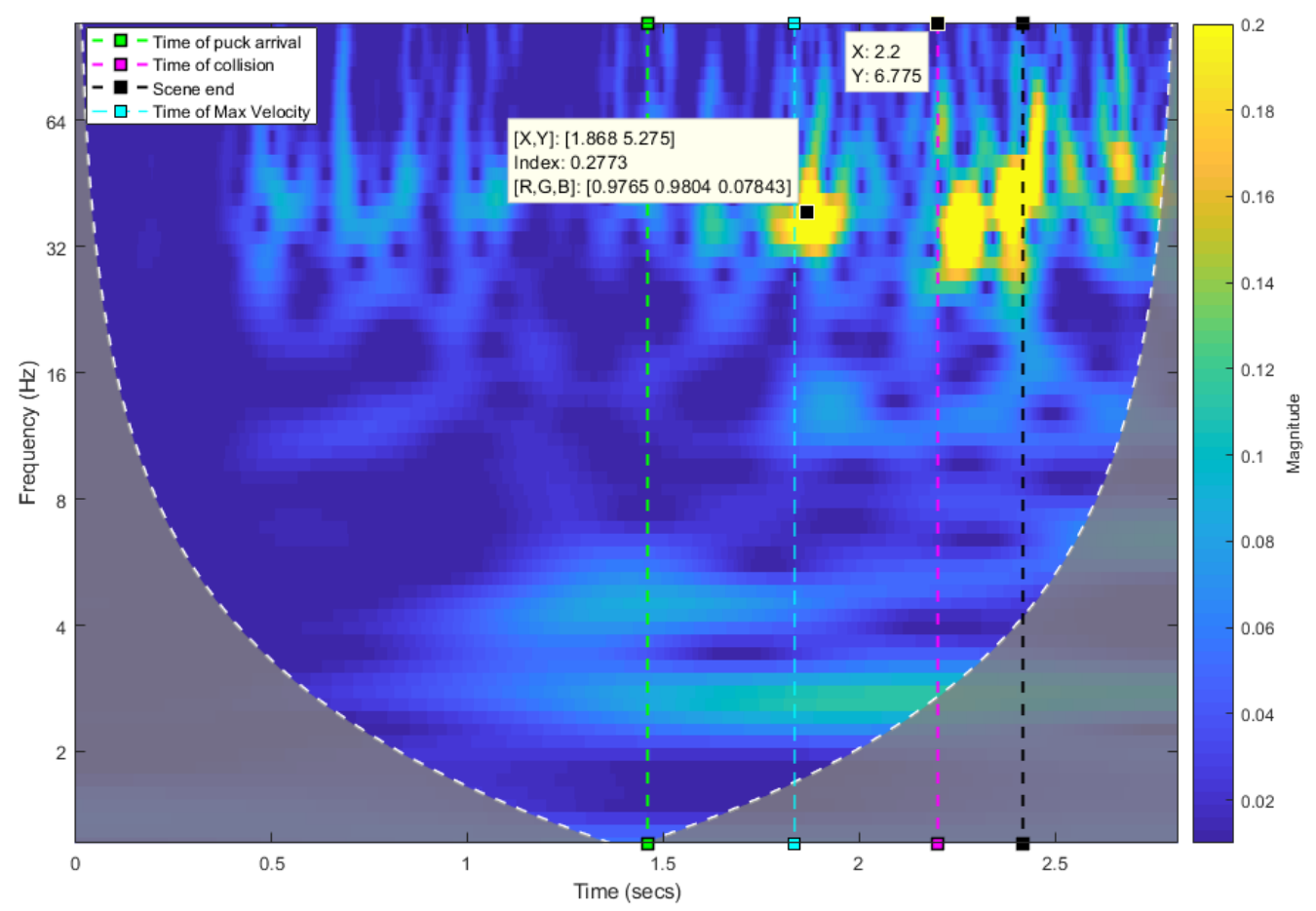

Figure 4.12: CWT of an EMG Signal for a Non-concussed Participant

Contrast Figure 4.12 of a non-concussed participant's response to a concussed participant's response in Figure 4.13. It is clear that it takes more time for the concussed participant to respond to the oncoming challenge. This delay could be a result of spatial disorientation where the participant is unaware of where the challenge is coming from, or it could be a cognitive delay in information processing as a result of the concussion [2, 30]. However, no conclusions can be made for one scene from two participants. This will be discussed in more detail in Chapter 6. 


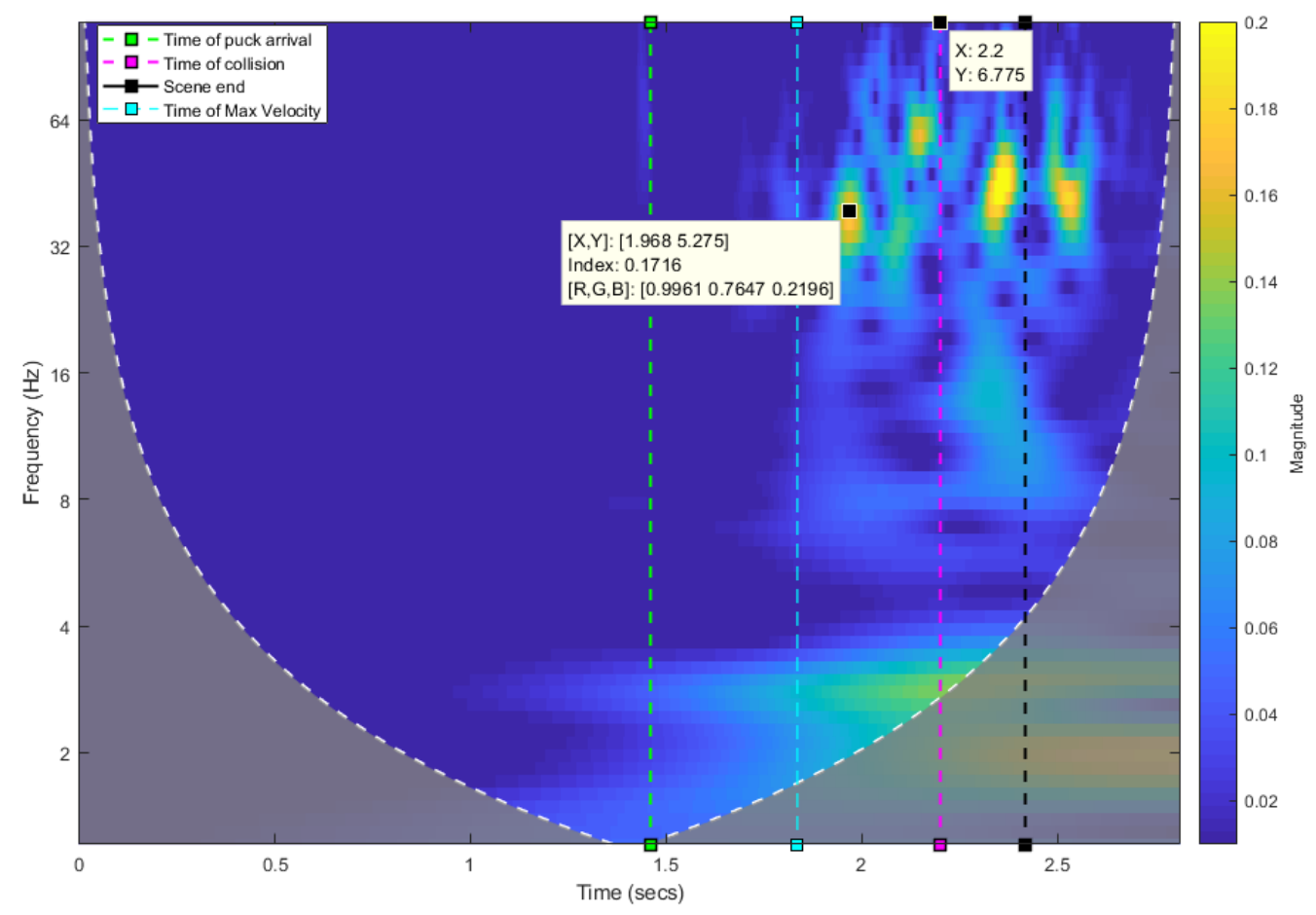

Figure 4.13: CWT of an EMG Signal for a Concussed Participant

Another observable difference between the two participants is the frequency response. The rectus femoris of the non-concussed participant is more active throughout the entire duration of the scene, and reaches a maximum before the opposing player reaches terminal velocity. This maximum contraction is maintained for approximately $200 \mathrm{~ms}$, and is again observed between the time of collision and when the scene ends.

In contrast, the concussed participant shows more sporadic and narrower durations of frequency response, with maximum frequency response not lasting more than $50 \mathrm{~ms}$.

\subsubsection{Principal Component Analysis}

PCA is an unsupervised regression algorithm used in pattern recognition. More specifically it is an orthogonal linear transformation that transforms data into a new coordinate system such that the greatest variance by some projection of the data comes to lie on 
the first coordinate (called the first principal component), the second greatest variance on the second coordinate, etc [29]. PCA is used in identifying patterns in data by helping highlight their similarities and differences.

The researchers decided to use unsupervised learning because there are a lot of variations in the participants' biometric data that could cause drastic differences to the reaction times of both concussed and non-concussed participants. PCA was utilized as a first step to examine if there were any noticeable features in the reaction times. However, in the future, the use of Neural networks on large sets of data could shed light on more patterns within the data.

Consider a data matrix $X$ that has 390 observations for 1 variable. The 1 variable represents one of the muscles, and the 390 observations represent the reactions times for each participant for each of the 30 scenes. The first step of PCA is to achieve zero empirical mean by subtracting the mean $\bar{X}$ :

$$
X-\bar{X}=X-\frac{\sum_{i=1}^{n} X_{i}}{n}
$$

where $X$ is a 390 by 1 matrix of reaction times and $\mathrm{n}$ is the number of observations.

The second step is to calculate the covariance matrix between the muscles, recall that the covariance of a column of $X$ with itself is simply the variance. The covariance is mathematically defined as follows:

$$
\operatorname{cov}(X, Y)=\frac{\sum_{i=1}^{n}\left(X_{i}-\bar{X}\right)\left(Y_{i}-\bar{Y}\right)}{n-1}
$$

where Y represents another one of the six muscles.

This process is repeated with all six muscles until a covariance matrix is calculated. A positive covariance indicates that both $X$ and $Y$ increase together, a negative covariance indicates that as one of the dimensions increases, the other decreases.

The eigenvectors and eigenvalues of the covariance matrix are then calculated where the eigenvectors represent the new transformed basis of the data. The largest eigenvector represents the first principal component and represents the largest variance in the data, the second principal component represents the second largest eigenvector and the second largest variance in the data, etc.

Consider Figure 4.14 below of a sample data distribution. The vectors shown on the plot are the largest two vectors of the covariance matrix scaled by their eigenvalues, and shifted so that their tails are at the mean. 


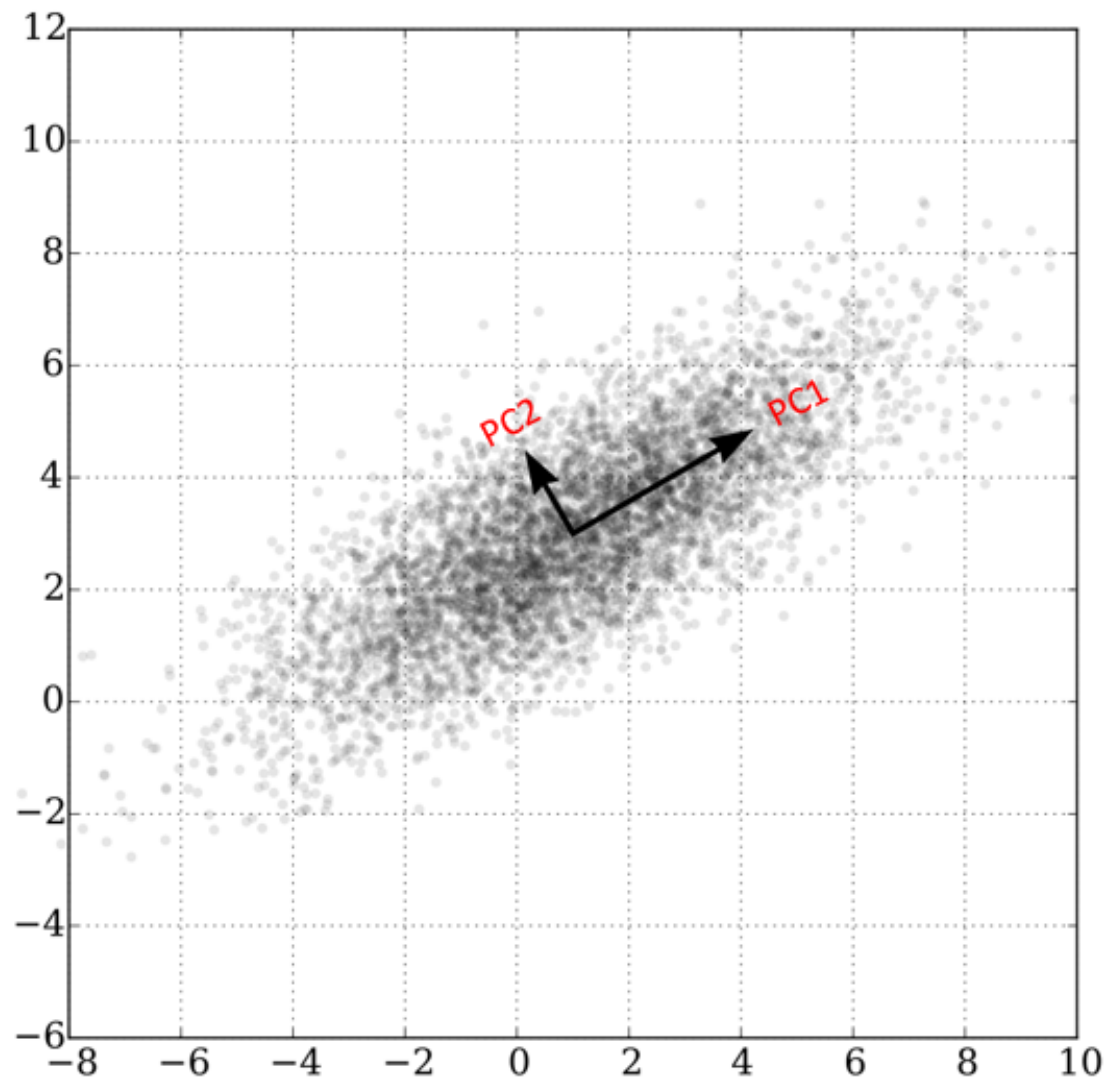

Figure 4.14: PCA Example

The largest variance in the data set lies along the vector labelled 'PC1' whereas the second largest variance lies along the vector labelled 'PC2' as seen in Figure 4.14. These two vectors are the principal components and form the new transformed coordinate system. 


\subsection{Summary of Results}

\section{Reaction Times}

Data was collected from 13 participants. They were divided into 3 groups:

- CE - Concussed \& Experienced - 6 participants

- NCE - Not Concussed \& Experienced - 5 participants

- NCNE - Not Concussed \& Not Experienced - 2 participants

Players who had suffered at least one concussion were placed in the CE group (there were no inexperienced players who had suffered concussions). Experienced players were those who had played ice hockey for at least 10 years.

Figure 4.15 shows the output of the transformed data after the application of PCA. The total variance is divided across the 6 principal components as follows:

- Principal component 1 - $47 \%$

- Principal component 2 - $14 \%$

- Principal component 3 - $12 \%$

- Principal component $4-10 \%$

- Principal component 5 - 9\%

- Principal component $6-7 \%$

It is apparent that principal component 1 accounts for approximately $50 \%$ of the variance in the data. Referring to Figure 4.15, principal component 1 displays the weighted reaction times of the 6 different muscles labelled in the figure.

All six variables are represented in the biplot by a vector, and the direction and length of the vector indicate how each variable contributes to the two principal components in the plot. For example, the first principal component, which is on the horizontal axis, has positive coefficients for all six variables. The second principal component, which is on the vertical axis, has negative coefficients for the right tibialis anterior, the left rectus 
femoris, the left tibialis anterior, and the left vastus medialis. However this component has a positive coefficient for the right rectus femoris and the right vastus medialis.

A boxplot of principal component 1 could shed light onto the different reaction times in the 3 different groups. However, principal component 2 has no physiological significance since it represents the differential reaction time between different muscles.

Furthermore, principal component 2 accounts for only $14 \%$ of the total variance. This is not very significant considering that the variances depicted by the remaining 4 components are very close. There is therefore no added benefit in visualizing the data in 3D by including principal component 3 . 


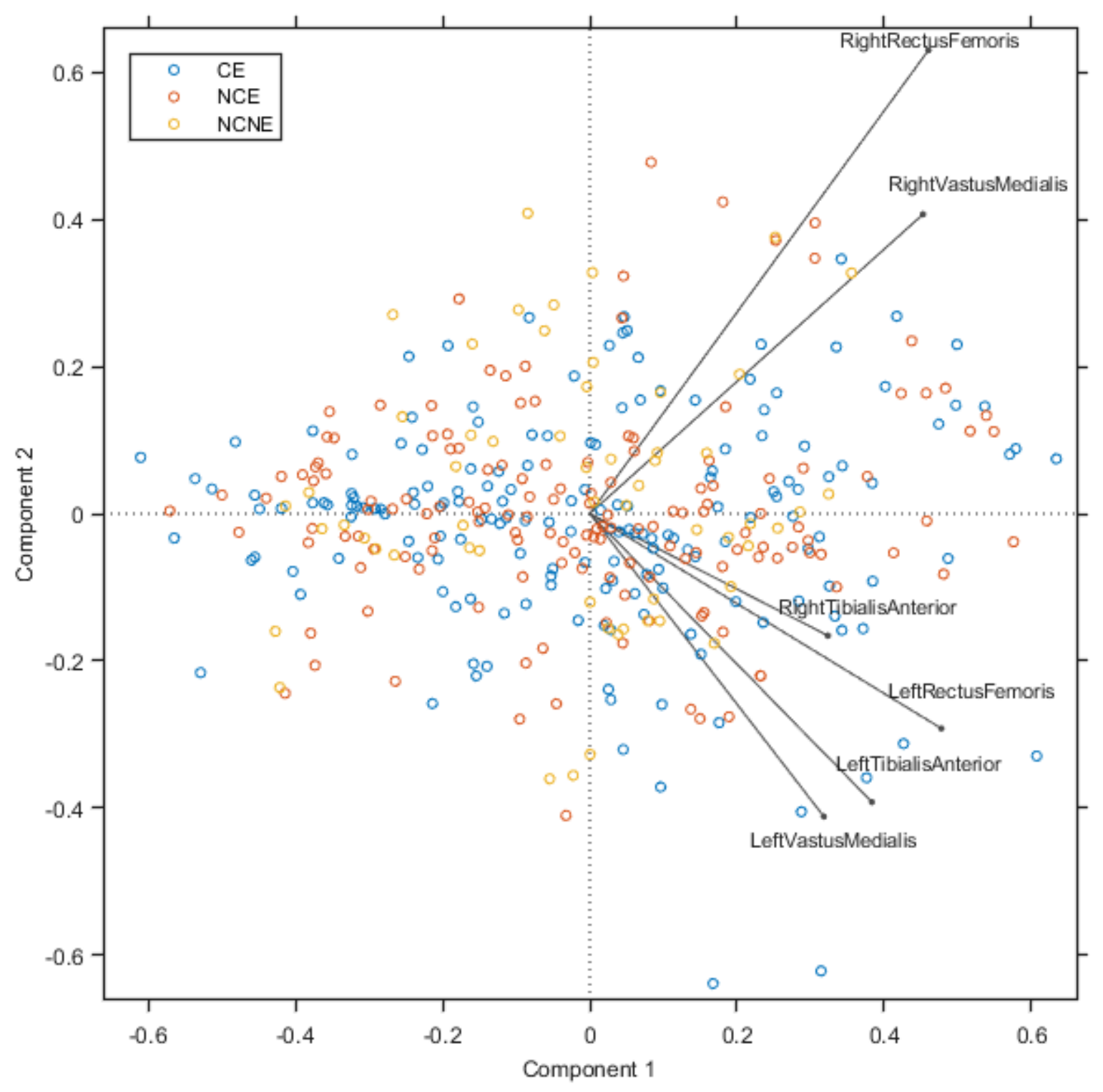

Figure 4.15: PCA for the EMG Data Set

Figure 4.16 displays the boxplot for principal component 1 . The 3 distinct groups are displayed separately. The data markers on the plot give information regarding the interquartile range. It appears that there is very little difference between the average reaction times for the three different groups. No conclusion can be formed from the reaction 
times of concussed and non-concussed participants.

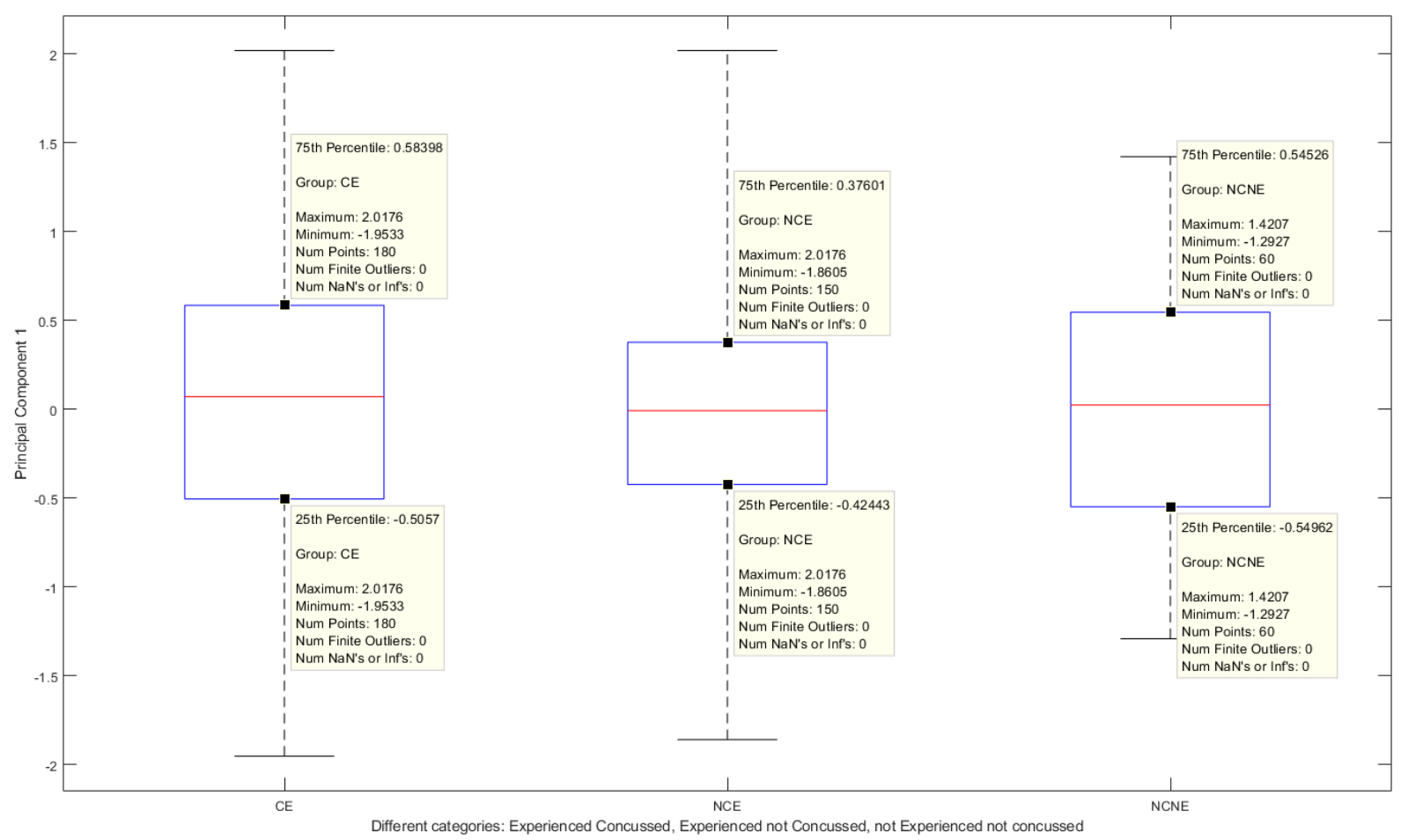

Figure 4.16: Principal Component 1 Boxplot 


\section{Head Directionality}

Head directionality (Yaw) was determined for all participants for scenes 12 and 13. These scenes, shown in Figure 4.17 and Figure 4.18, represent a particularly difficult scenario for any hockey player. The participant is in the center (marked by a yellow circle) with two opposing players (marked by a red X) and four teammates (marked by a green X). The puck (marked by the direction of a green arrow) is passed towards the participant as one of the opposing players (marked by the direction of a red arrow) charges towards the participant.



Figure 4.17: Scene 12 


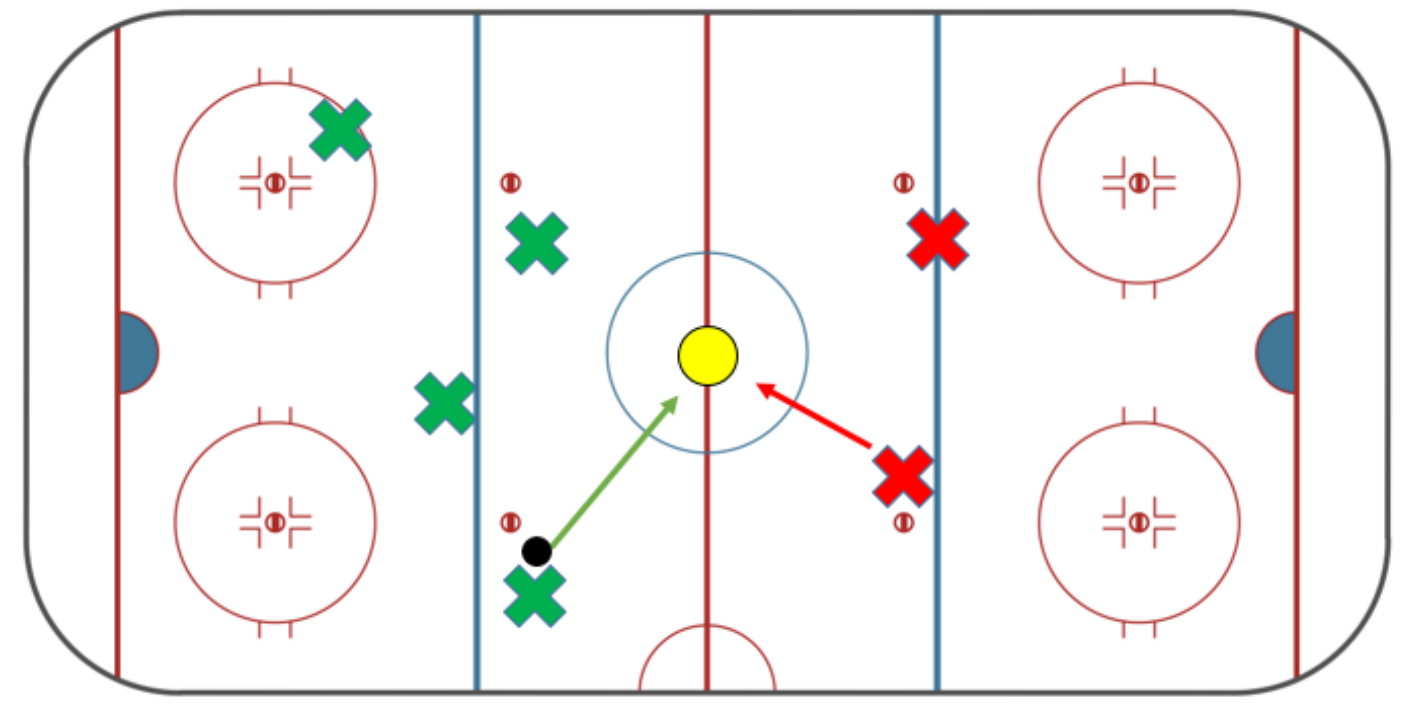

Figure 4.18: Scene 13

Figure 4.19 shows the head directionality of all 13 participants for Scene 12. Note that the puck arrival and player collision occur at the same time. 




Figure 4.19: Scene 12 responses

It may appear that some participants show almost 0 movement between the terminal velocity of the opposing player and the time of collision. However, this is not indicative of a concussion.

Consider the boxplot in Figure 4.20 where the 3 distinct groups are labelled (Concussed Experienced, Not Concussed Experienced, and Not Concussed Not Experienced). This boxplot contains the head directionality for all participants for both scenes 12 and 13 . 


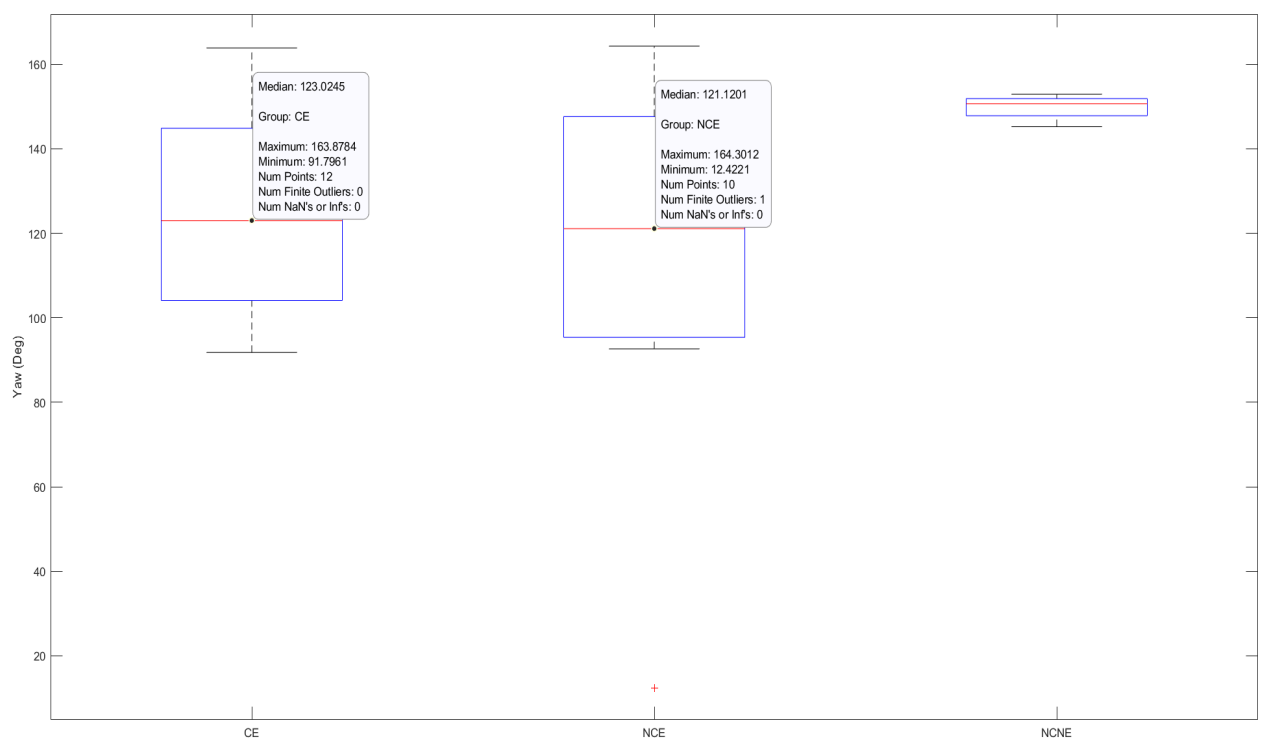

Figure 4.20: Scenes 12 and 13 Boxplot

While the average angle of the inexperienced and non-concussed group seems to be drastically different from the other two groups, it is important to note that the group is comprised of only 2 participants and therefore is not indicative of a true response. This group was isolated so as not to affect the responses of the experienced participants.

The directionality between experienced concussed and non-concussed participants is centered around the same mean angle with a slight deviation of approximately 2 degrees. The distribution is the same for both groups suggesting that there might not be an observable difference between participants with a history of concussion and non-concussed participants. 


\section{Chapter 5}

\section{Conclusions}

The purpose of this thesis was to develop a quantitative metric by which concussions can be assessed, a secondary goal was to use visual aids to assist physicians and clinicians in making assessments.

In Chapters 1 and 2, the problem with concussions was investigated in detail. Concussions are elusive and include a wide variety of symptoms that range from trivial to detrimental. Classifying and diagnosing concussions is a difficult task yet it is of utter importance since the well-being of several athletes rests on precise diagnosis.

Chapter 3 described the methodology taken to create diagnostic tools. A 360 degree camera and microphone were used to collect real-life hockey scenarios that involve dualtask management. The scenarios involve the hockey puck being passed towards the camera and an ice hockey player charging towards the camera. This data was then processed and fitted into Unity game engine so that it could be played back in a VR environment. The use of VR along with synthetic ice provided an immersive environment where the responses of hockey players could be collected in response to a virtual stimulus that posed no physical risk. EMG was collected alongside motion capture data in an attempt to capture the reaction time of participants and the directionality of their heads.

Chapter 4 discussed some signal processing techniques for filtering and extracting relevant information from the raw EMG data. The use of PCA proved useful in helping analyze reaction times of different groups, and boxplots of the first two principal components provided sufficient information to infer some observations.

The results section was divided in two, reaction time, and head directionality. No comment can be made regarding the statistical significance of data since there weren't 
enough data points available for the use of a T-test. There was no observable difference between any of the three groups when it came to reaction time as observed in Figure 4.16. The interquartile range of the experienced non-concussed group was slightly narrower than the concussed group; however, the overall range was the same, and the difference was insignificant. The inexperienced and non-concussed group involved only two participants, which made it difficult to draw any results based on their responses. Regardless, for reaction time, there was no difference between their performance and the other two group's performance.

It is difficult to make a claim regarding the directionality observed in Figure 4.20. Though the range is larger for the non-concussed participants, the data points only comprise one scene and are therefore not indicative of any differences between the groups.

No claims can be made regarding the difference in responses between concussed and non-concussed hockey players. There were several different factors that could have made an impact such as, time since last concussion, recovery period, severity of the concussion, and if any formal therapy was received. The most recent concussion that one of the participants had suffered was six years prior to undergoing the experiment, which should not have had any effect on the participant [21].

While it could be the case that there is no difference in the reaction time and directionality of non-concussed and concussed hockey players, more data needs to be collected. Of interest would be to collect data from recently concussed participants ( $<1$ month) over several different sessions to monitor their recovery. It is almost important to recall that the most recent concussion recorded was six years ago, therefore it is of no surprise that there is no difference between concussed and non-concussed responses.

Though the boxplots have demonstrated no difference between the three different groups overall. A case scenario between an experienced non-concussed participant and an experienced concussed participant shown in Figure 4.12 and Figure 4.13 demonstrated a significant difference in their reaction times and their time frequency plots. The concussed participant was slower and their rectus femoris was not activated for as long of a duration. Furthermore, their head directionality shown for one scene in Figure 3.16 and Figure 3.17 also showed a significant difference. The non-concussed participant showed great variability in their directionality when compared to the concussed participant. These observations would confirm the hypotheses of this thesis. However, no conclusion can be drawn from a case scenario.

The methodology developed in this experiment proved to be immersive. The participants found themselves engaged and responsive to the external stimulus of a virtual body-check. The VR environment proved to be safe for testing. This was validated by 
comparing participant inputs from the Acute Concussion Evaluation (ACE) and SCAT surveys to their responses during SAGAT inquiries regarding any dizziness, nausea, or any general discomfort. Furthermore, the pain scale was not utilized once during the experiment.

The use of the CWT to generate a time frequency plot proved to be useful in visualizing the response times of the participants while monitoring the frequency response of the muscle of interest. This visual aid can be used by physicians and clinicians to draw information on the response time as well as the activation of the muscle of interest.

Finally, though no conclusion can be drawn from the results. The experimental procedure developed proved to be very useful, and with the a larger participant sample size can provide some great insight. 


\section{Chapter 6}

\section{Future Work \& Current Limitations}

This section discusses several improvements that could be made to the experimental procedure and design, and suggests future steps to be taken by the next set of researchers.

\subsection{Participant Data Collection}

Collecting data from hockey players with a history of concussions proved to be challenging. Ideally in the future, the next set of researchers would partner with a sports rehabilitation clinic in order to collect more data. This would help overcome the limited sample size in this thesis. Furthermore, collecting responses from recently concussed participants can provide great insight into the recovery time from concussions.

\subsection{Wireless Approach}

During the first draft of the experimental procedure, a wired EMG Hub was utilized for EMG data collection. However, the research team quickly discovered the limitations of a wired system, the interference caused by EMG electrodes falling off, and by the interaction between the EMG electrode cables and the serial wire attached to the USB input of the computer. The research team iterated to another EMG Hub with Bluetooth capability. However, Bluetooth provided a $250 \mathrm{~Hz}$ sampling rate which is lower than ideal for EMG measurements, and therefore while the Bluetooth option is still available, the research team had decided to collect data on an SD card directly attached to the EMG OpenBCI 
data collection board. This option allows the data to be sampled at $1 \mathrm{KHz}$. Unfortunately, while the first 2 sets of data from the SD card were stored without any issue, the remaining 11 sets of data were corrupted. This left the researchers with no option but to use data sampled at $250 \mathrm{~Hz}$.

The first improvement would be to purchase the OpenBCI wireless shield which allows for wireless streaming at a $1 \mathrm{KHz}$ sampling rate. The second improvement would be to purchase a wireless head-mounted display such as the HTC Vive to avoid the entanglement and interference of cables. Another added benefit of a new head-mounted display is that the latest versions provide eye tracking which would greatly help with identifying directionality. One of the issues faced with collecting IR motion capture data is that participants with different play styles react differently and the directionality of the head could be misleading as the eyes could be facing another direction.

\subsection{EMG Improvements}

The quadriceps femoris muscle group minus the vastus lateralis was monitored throughout the experiment. Including the vastus lateralis would allow for a complete picture of the anterior legs muscles. Furthermore posterior muscles such as the hamstring should also be monitored in the future to check for cocontraction.

\subsection{ECG and EEG Data Collection}

In the initial draft of the experiment, there was interest in collecting both ECG and EEG data, but they were not pursued.

It would be useful if future researchers carried out EEG measurements either using the already purchased OpenBCI Hub or with a different setup. Research has already been done on concussion testing with EEG data. This further gives the researcher more biometric data that can be used in conjunction with what is already being collected to give a more accurate diagnosis tool. ECG has not been utilized in concussion testing, however, since the technology for ECG data collection is becoming significantly more abundant, it might be worthwhile to gather ECG data for classification purposes. 


\subsection{Improved Motion Capture Technology}

Optitrack Tracking Tools has been retired and proved difficult to work with. Optitrack's new Motive software provides a great platform that can accelerate the process of data collection and analysis. If more precise measurements are to be taken then it might be worthwhile to consider investing in more advance motion capture technology such as Qualisys [27].

\subsection{Theoretical Improvements}

More research needs to be put into the type of CWT and DWT used. The ones incorporated in this experiment were based off the works of previous researchers, however, improvements could still be made. This will help ensure that no useful information is lost when denoising or when computing the time frequency analysis of an EMG signal.

Further signal processing techniques should be explored. PCA was used as a leeway into possibly identifying patterns based off the variance in the data. However, with increased participant sample size and larger data sets, more sophisticated and appropriate techniques should be utilized. Ideally, the use of supervised regression algorithms should be incorporated when comparing data from non-concussed to concussed participants. Further strategies could involve the use of neural networks to identify trend patterns in large data sets.

\subsection{Scenario Improvements}

One of the most common feedback comments from the participants was that they would have attempted to skate away to evade the hit if possible. The simulation did not allow for any translation but only rotation, which could have elicited an unnatural response from the participants. Furthermore, the scenes were greatly unrestricted in that the participants could have reacted in several different ways (such as by completing ignoring the puck and only focusing on the charging opposing player). This makes it possible to have several different 'correct' responses. In the future it would be ideal to restrict the scenarios in such a way where there would be only one correct response by which to evaluate all the participants.

Furthermore it might be beneficial to examine participant responses in a scene-by-scene basis in order to reduce the variability in the data set. Analyzing each scene separately 
while viewing EMG responses alongside head directionality might shed more light on the responses of concussed and non-concussed groups. 


\section{References}

[1] Brian W Benson, Willem H Meeuwisse, John Rizos, Jian Kang, and Charles J Burke. A prospective study of concussions among national hockey league players during regular season games: the nhl-nhlpa concussion program. Canadian Medical Association Journal, 183(8):905-911, 2011.

[2] Daniel M Bernstein. Information processing difficulty long after self-reported concussion. Journal of the International Neuropsychological Society, 8(5):673-682, 2002.

[3] Jeremy Besnard, Paul Richard, Frederic Banville, Pierre Nolin, Ghislaine Aubin, Didier Le Gall, Isabelle Richard, and Phillippe Allain. Virtual reality and neuropsychological assessment: The reliability of a virtual kitchen to assess daily-life activities in victims of traumatic brain injury. Applied Neuropsychology: Adult, 23(3):223-235, 2016.

[4] The Editors of Encyclopaedia Britannica. Quadriceps femoris muscle, Dec 2018.

[5] Jared M Bruce and Ruben J Echemendia. Concussion history predicts self-reported symptoms before and following a concussive event. Neurology, 63(8):1516-1518, 2004.

[6] Robert C Cantu. Recurrent athletic head injury: risks and when to retire. Clinics in sports medicine, 22(3):593-603, 2003.

[7] Robert C Cantu and Robert Voy. Second impact syndrome: a risk in any contact sport. The Physician and Sportsmedicine, 23(6):27-34, 1995.

[8] J Cegalis and J Bowlin. Vigil: software for the assessment of attention. Nashua, NH: Forthought, 1991.

[9] Alison Cernich, Dennis Reeves, Wenyu Sun, and Joseph Bleiberg. Automated neuropsychological assessment metrics sports medicine battery. Archives of Clinical Neuropsychology, 22(Suppl_1):S101-S114, 2007. 
[10] Esther Y Chin, Lindsay D Nelson, William B Barr, Paul McCrory, and Michael A McCrea. Reliability and validity of the sport concussion assessment tool-3 (scat3) in high school and collegiate athletes. The American journal of sports medicine, 44(9):2276-2285, 2016.

[11] Liu Chun-Lin. A tutorial of the wavelet transform. NTUEE, Taiwan, 2010.

[12] Rodney L Coldren, Mark P Kelly, Robert V Parish, Michael Dretsch, and Michael L Russell. Evaluation of the military acute concussion evaluation for use in combat operations more than 12 hours after injury. Military medicine, 175(7):477-481, 2010.

[13] National Research Council, Committee on Sports-Related Concussions in Youth, et al. Sports-related concussions in youth: improving the science, changing the culture. National Academies Press, 2014.

[14] Tracey Covassin and Robert J Elbin. The cognitive effects and decrements following concussion. Open access journal of sports medicine, 1:55, 2010.

[15] Tracey Covassin, C Buz Swanik, Michael Sachs, Zebulon Kendrick, Phillip Schatz, Eric Zillmer, and Catherine Kaminaris. Sex differences in baseline neuropsychological function and concussion symptoms of collegiate athletes. British journal of sports medicine, 40(11):923-927, 2006.

[16] Jeffrey P Cuthbert, Kristi Staniszewski, Kaitlin Hays, Don Gerber, Audrey Natale, and Denise Odell. Virtual reality-based therapy for the treatment of balance deficits in patients receiving inpatient rehabilitation for traumatic brain injury. Brain injury, 28(2):181-188, 2014.

[17] Marie N Dahdah, Monica Bennett, Purvi Prajapati, Thomas D Parsons, Erin Sullivan, and Simon Driver. Application of virtual environments in a multi-disciplinary day neurorehabilitation program to improve executive functioning using the stroop task. NeuroRehabilitation, 41(4):721-734, 2017.

[18] Assaf Y Dvorkin, Milan Ramaiya, Eric B Larson, Felise S Zollman, Nancy Hsu, Sonia Pacini, Amit Shah, and James L Patton. A virtually minimal visuo-haptic training of attention in severe traumatic brain injury. Journal of neuroengineering and rehabilitation, 10(1):92, 2013.

[19] Ruben J Echemendia, Willem Meeuwisse, Paul McCrory, Gavin A Davis, Margot Putukian, John Leddy, Michael Makdissi, S John Sullivan, Steven P Broglio, Martin 
Raftery, et al. The sport concussion assessment tool 5th edition (scat5): background and rationale. Br J Sports Med, 51(11):848-850, 2017.

[20] Mica R Endsley. Toward a theory of situation awareness in dynamic systems. Human factors, 37(1):32-64, 1995.

[21] Massimo Gentilini, Paolo Nichelli, RUDOLF Schoenhuber, Paolo Bortolotti, Luigi Tonelli, Angelo Falasca, and Gian A Merli. Neuropsychological evaluation of mild head injury. Journal of Neurology, Neurosurgery \& Psychiatry, 48(2):137-140, 1985.

[22] Neal R Glaviano, Shari Benson, Howard P Goodkin, Donna K Broshek, and Susan Saliba. Baseline scat2 assessment of healthy youth student-athletes: preliminary evidence for the use of the child-scat3 in children younger than 13 years. Clinical Journal of Sport Medicine, 25(4):373-379, 2015.

[23] Matthew Gorman, Suzanne Hecht, Andrew Samborski, Scott Lunos, Steven Elias, and Steven D Stovitz. Scat3 assessment of non-head injured and head injured athletes competing in a large international youth soccer tournament. Applied Neuropsychology: Child, 6(4):364-368, 2017.

[24] Yang Guang-ying and Luo Zhi-zeng. Surface electromyography disposal based on the method of wavelet de-noising and power spectrum. In 2004 International Conference on Intelligent Mechatronics and Automation, 2004. Proceedings., pages 896-900. IEEE, 2004.

[25] Kevin M Guskiewicz, Johna Register-Mihalik, Paul McCrory, Michael McCrea, Karen Johnston, Michael Makdissi, Jiří Dvořák, Gavin Davis, and Willem Meeuwisse. Evidence-based approach to revising the scat2: introducing the scat3. $\mathrm{Br} J$ Sports Med, 47(5):289-293, 2013.

[26] Jr JA Isear, Jeff C Erickson, and Teddy W Worrell. Emg analysis of lower extremity muscle recruitment patterns during an unloaded squat. Medicine and science in sports and exercise, 29(4):532-539, 1997.

[27] Alexander Refsum Jensenius, Kristian Nymoen, Ståle Andreas van Dorp Skogstad, and Arve Voldsund. A study of the noise-level in two infrared marker-based motion capture systems. 2012.

[28] Ian Jolliffe. Principal component analysis. Springer, 2011. 
[29] IT Jolliffe. Mathematical and statistical properties of population principal components. Principal component analysis, pages 10-28, 2002.

[30] Barry D Jordan, Eric JT Matser, Robert D Zimmerman, and Tania Zazula. Sparring and cognitive function in professional boxers. The Physician and sportsmedicine, 24(5):87-98, 1996.

[31] James P Kelly, John S Nichols, Christopher M Filley, Kevin O Lillehei, David Rubinstein, and BK Kleinschmidt-DeMasters. Concussion in sports: guidelines for the prevention of catastrophic outcome. Jama, 266(20):2867-2869, 1991.

[32] Chad Killam, Robin L Cautin, and Anthony C Santucci. Assessing the enduring residual neuropsychological effects of head trauma in college athletes who participate in contact sports. Archives of clinical neuropsychology, 20(5):599-611, 2005.

[33] James Kissick and Karen M Johnston. Return to play after concussion: principles and practice. Clinical Journal of Sport Medicine, 15(6):426-431, 2005.

[34] Jens Krepela. German soccer lags behind on concussion-risk awareness — dw 02.01.2017, Jan 2017.

[35] Vineet Kumar and Kashinath Murmu. Chapter 3: The uncertainty principle \& timebandwidth product.

[36] Jonathan M Lilly and Sofia C Olhede. Higher-order properties of analytic wavelets. IEEE Transactions on Signal Processing, 57(1):146-160, 2009.

[37] Jonathan M Lilly and Sofia $\mathrm{C}$ Olhede. On the analytic wavelet transform. IEEE Transactions on Information Theory, 56(8):4135-4156, 2010.

[38] Jonathan M Lilly and Sofia C Olhede. Generalized morse wavelets as a superfamily of analytic wavelets. IEEE Transactions on Signal Processing, 60(11):6036-6041, 2012.

[39] Mark R Lovell, Grant L Iverson, Michael W Collins, Kenneth Podell, Karen M Johnston, Dustin Pardini, Jamie Pardini, John Norwig, and Joseph C Maroon. Measurement of symptoms following sports-related concussion: reliability and normative data for the post-concussion scale. Applied neuropsychology, 13(3):166-174, 2006.

[40] DL Maddocks, GD Dicker, and MM Saling. The assessment of orientation following concussion in athletes. Clinical journal of sport medicine : official journal of the Canadian Academy of Sport Medicine, 5(1):3235, 1995. 
[41] Stephane G Mallat. A theory for multiresolution signal decomposition: the wavelet representation. IEEE Transactions on Pattern Analysis 83 Machine Intelligence, (7):674-693, 1989.

[42] Frederic H. Martini, Judi Lindsley Nath, Edwin F. Bartholomew, and Kathleen Welch. Fundamentals of anatomy $\& 3$ physiology. Interactive physiology. Pearson, 2012.

[43] S Mathur, JJ Eng, and DL MacIntyre. Reliability of surface emg during sustained contractions of the quadriceps. Journal of Electromyography and Kinesiology, 15(1):102$110,2005$.

[44] Michael McCrea, James P Kelly, Christopher Randolph, Jon Kluge, Edward Bartolic, George Finn, and Brian Baxter. Standardized assessment of concussion (sac): on-site mental status evaluation of the athlete. The Journal of head trauma rehabilitation, 13(2):27-35, 1998.

[45] Paul McCrory, Willem H Meeuwisse, Ruben J Echemendia, Grant L Iverson, Jiří Dvořák, and Jeffrey S Kutcher. What is the lowest threshold to make a diagnosis of concussion? Br J Sports Med, 47(5):268-271, 2013.

[46] TM McMillan, P McSkimming, J Wainman-Lefley, LM Maclean, J Hay, A McConnachie, and W Stewart. Long-term health outcomes after exposure to repeated concussion in elite level: rugby union players. J Neurol Neurosurg Psychiatry, 88(6):505-511, 2017.

[47] George S Murley, Hylton B Menz, Karl B Landorf, and Adam R Bird. Reliability of lower limb electromyography during overground walking: a comparison of maximaland sub-maximal normalisation techniques. Journal of biomechanics, 43(4):749-756, 2010 .

[48] Sarah Nichols and Harshada Patel. Health and safety implications of virtual reality: a review of empirical evidence. Applied ergonomics, 33(3):251-271, 2002.

[49] Pierre Nolin, Annie Stipanicic, Mylène Henry, Christian C Joyal, and Philippe Allain. Virtual reality as a screening tool for sports concussion in adolescents. Brain injury, 26(13-14):1564-1573, 2012.

[50] Sofia C Olhede and Andrew T Walden. Generalized morse wavelets. IEEE Transactions on Signal Processing, 50(11):2661-2670, 2002. 
[51] Linda Papa, Steven A Robicsek, Gretchen M Brophy, Kevin KW Wang, H Julia Hannay, Shelley Heaton, Ilona Schmalfuss, Andrea Gabrielli, Ronald L Hayes, and Claudia S Robertson. Temporal profile of microtubule-associated protein 2: a novel indicator of diffuse brain injury severity and early mortality after brain trauma. Journal of neurotrauma, 35(1):32-40, 2018.

[52] Tonya M Parker, Louis R Osternig, Paul Van Donkelaar, and Li-shan Chou. Gait stability following concussion. Medicine $\& 5$ Science in Sports $\&$ Exercise, 38(6):10321040, 2006.

[53] Tonya M Parker, Louis R Osternig, Paul van Donkelaar, and Li-Shan Chou. Balance control during gait in athletes and non-athletes following concussion. Medical engineering \& physics, 30(8):959-967, 2008.

[54] Thomas D Parsons. Neuropsychological assessment using virtual environments: enhanced assessment technology for improved ecological validity. In Advanced computational intelligence paradigms in healthcare 6. Virtual reality in psychotherapy, rehabilitation, and assessment, pages 271-289. Springer, 2011.

[55] Angkoon Phinyomark, Chusak Limsakul, and Pornchai Phukpattaranont. An optimal wavelet function based on wavelet denoising for multifunction myoelectric control. In 2009 6th International Conference on Electrical Engineering/Electronics, Computer, Telecommunications and Information Technology, volume 2, pages 1098-1101. IEEE, 2009.

[56] Angkoon Phinyomark, Chusak Limsakul, and Pornchai Phukpattaranont. Application of wavelet analysis in emg feature extraction for pattern classification. Measurement Science Review, 11(2):45-52, 2011.

[57] Megan HW Preece, Mark S Horswill, and Gina M Geffen. Driving after concussion: the acute effect of mild traumatic brain injury on drivers' hazard perception. Neuropsychology, 24(4):493, 2010.

[58] Margot Putukian, Ruben Echemendia, Annegret Dettwiler-Danspeckgruber, Tawny Duliba, Jared Bruce, John L Furtado, and Murali Murugavel. Prospective clinical assessment using sideline concussion assessment tool-2 testing in the evaluation of sportrelated concussion in college athletes. Clinical journal of sport medicine, 25(1):36-42, 2015 . 
[59] Mamun Bin Ibne Reaz, MS Hussain, and Faisal Mohd-Yasin. Techniques of emg signal analysis: detection, processing, classification and applications. Biological procedures online, 8(1):11, 2006.

[60] Olivier Rioul and Martin Vetterli. Wavelets and signal processing. IEEE signal processing magazine, 8(ARTICLE):14-38, 1991.

[61] Nicolas Robitaille, Philip L Jackson, Luc J Hébert, Catherine Mercier, Laurent J Bouyer, Shirley Fecteau, Carol L Richards, and Bradford J McFadyen. A virtual reality avatar interaction (vrai) platform to assess residual executive dysfunction in active military personnel with previous mild traumatic brain injury: proof of concept. Disability and Rehabilitation: Assistive Technology, 12(7):758-764, 2017.

[62] Kathryn J Schneider, Carolyn A Emery, Jian Kang, Geoff M Schneider, and Willem H Meeuwisse. Examining sport concussion assessment tool ratings for male and female youth hockey players with and without a history of concussion. British journal of sports medicine, 44(15):1112-1117, 2010.

[63] Mark R Schulz, Stephen W Marshall, Frederick O Mueller, Jingzhen Yang, Nancy L Weaver, William D Kalsbeek, and J Michael Bowling. Incidence and risk factors for concussion in high school athletes, north carolina, 1996-1999. American journal of epidemiology, 160(10):937-944, 2004.

[64] Nadia Shehata, JP Wiley, Schad Richea, Brian W Benson, Lucas Duits, and WH Meeuwisse. Sport concussion assessment tool: baseline values for varsity collision sport athletes. British journal of sports medicine, 43(10):730-734, 2009.

[65] Brij N Singh and Arvind K Tiwari. Optimal selection of wavelet basis function applied to ecg signal denoising. Digital signal processing, 16(3):275-287, 2006.

[66] Lauren H Smith and Levi J Hargrove. Comparison of surface and intramuscular emg pattern recognition for simultaneous wrist/hand motion classification. In 2013 35th annual international conference of the IEEE engineering in medicine and biology society $(E M B C)$, pages 4223-4226. IEEE, 2013.

[67] Elizabeth Teel, Michael Gay, Brian Johnson, and Semyon Slobounov. Determining sensitivity/specificity of virtual reality-based neuropsychological tool for detecting residual abnormalities following sport-related concussion. Neuropsychology, 30(4):474, 2016. 
[68] Christopher Torrence and Gilbert P Compo. A practical guide to wavelet analysis. Bulletin of the American Meteorological society, 79(1):61-78, 1998.

[69] Tamara C Valovich McLeod, R Curtis Bay, Kenneth C Lam, and Anikar Chhabra. Representative baseline values on the sport concussion assessment tool 2 (scat2) in adolescent athletes vary by gender, grade, and concussion history. The American journal of sports medicine, 40(4):927-933, 2012.

[70] Mark P Wachowiak, Renata Wachowiak-Smolíková, Michel J Johnson, Dean C Hay, Kevin E Power, and F Michael Williams-Bell. Quantitative feature analysis of continuous analytic wavelet transforms of electrocardiography and electromyography. Philosophical Transactions of the Royal Society A: Mathematical, Physical and Engineering Sciences, 376(2126):20170250, 2018.

[71] Eric W Weisstein. Fourier series.

[72] Jackson Wright. The psychological effects of concussions on athletes.

[73] W Geoffrey Wright, Jane McDevitt, Ryan Tierney, F Jay Haran, Kwadwo Osei Appiah-Kubi, and Alex Dumont. Assessing subacute mild traumatic brain injury with a portable virtual reality balance device. Disability and rehabilitation, 39(15):1564$1572,2017$.

[74] WG Wright, RT Tierney, and J McDevitt. Visual-vestibular processing deficits in mild traumatic brain injury. Journal of Vestibular Research, 27(1):27-37, 2017.

[75] Elisa R Zanier, Tommaso Zoerle, Daniele Di Lernia, and Giuseppe Riva. Virtual reality for traumatic brain injury. Frontiers in neurology, 9:345, 2018. 
APPENDICES 
Appendix A

All the Different Hockey Scenes

\section{A.1 Scenario 1}

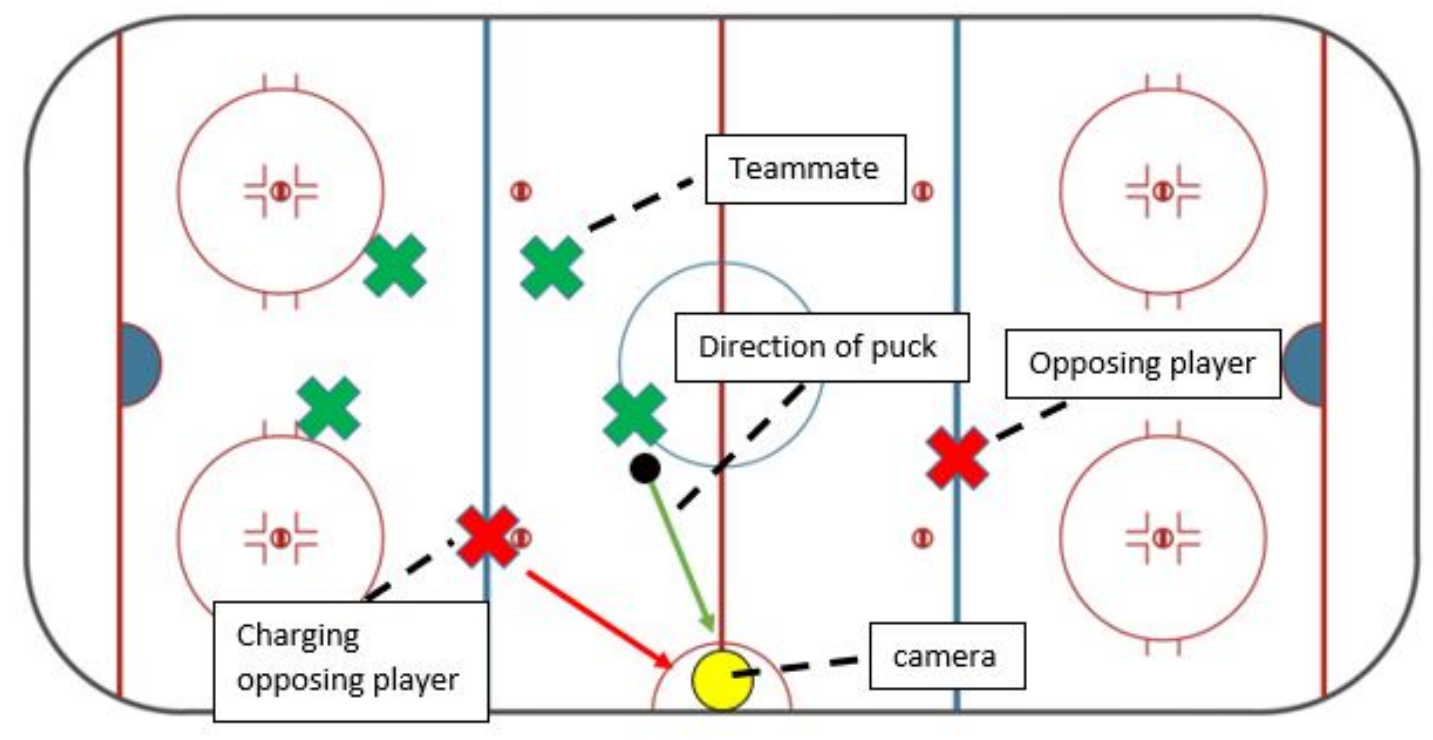

Figure A.1: Scene 1 


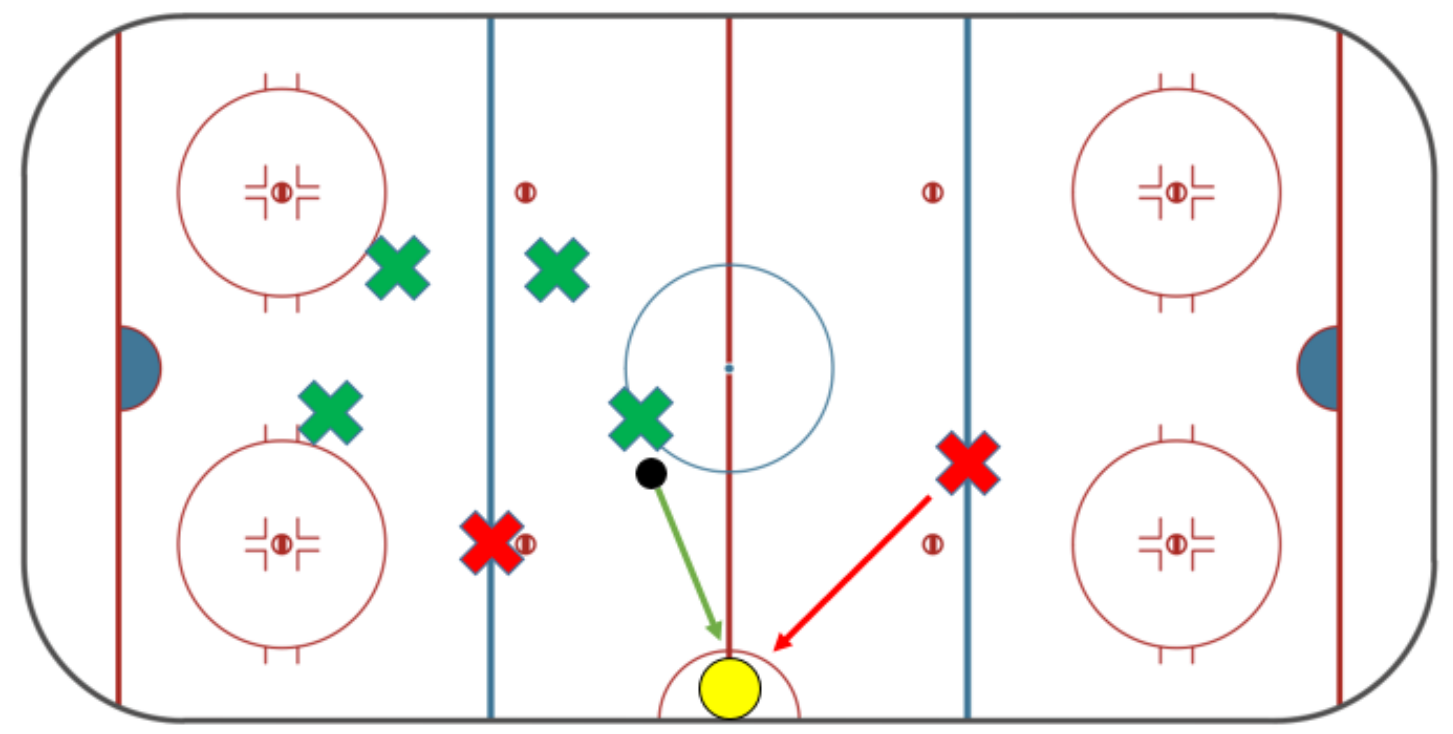

Figure A.2: Scene 2

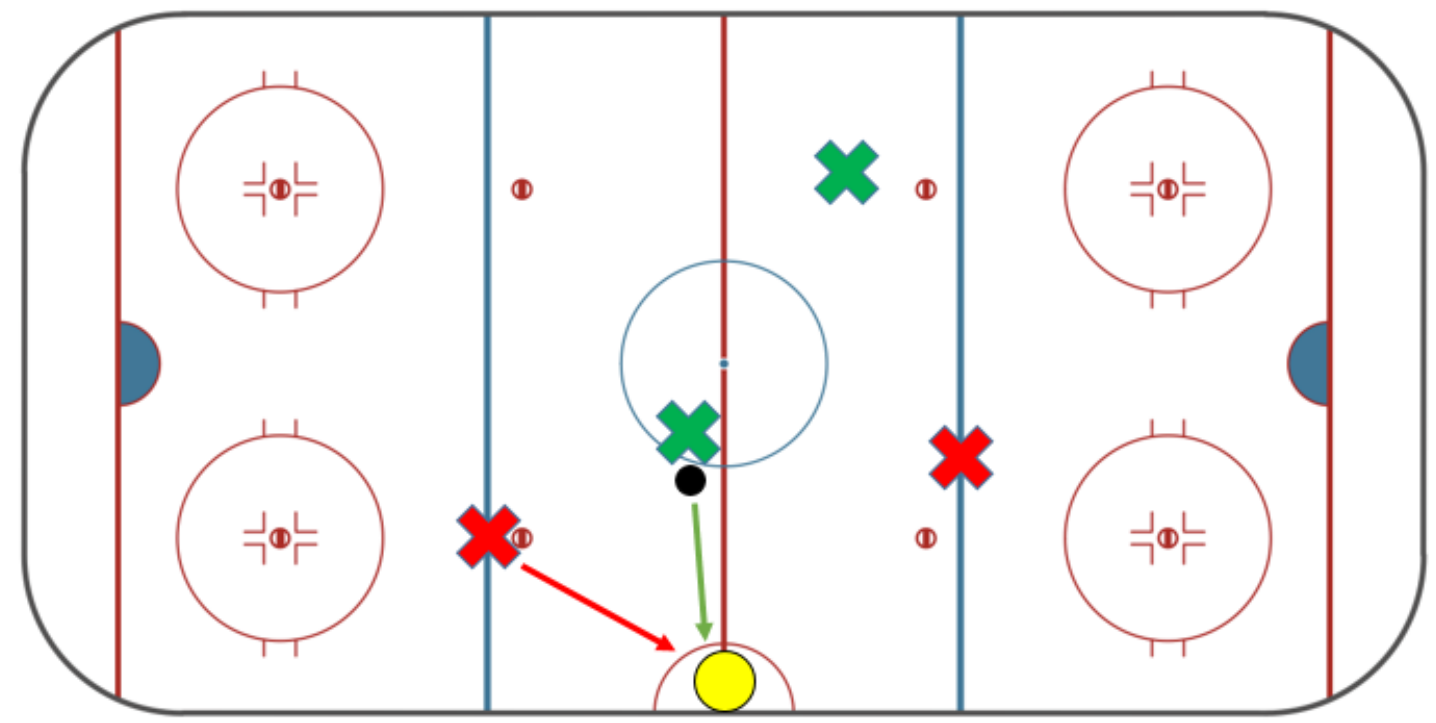

Figure A.3: Scene 3 


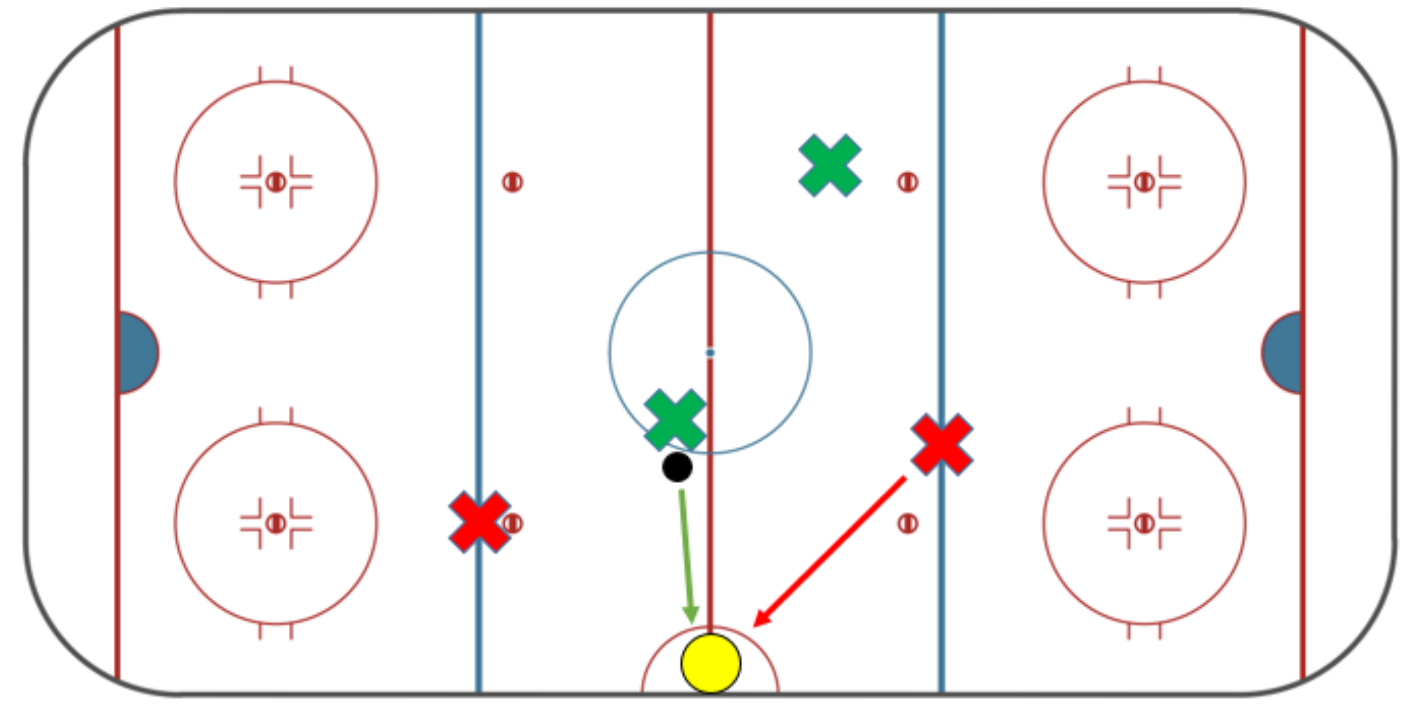

Figure A.4: Scene 4

\section{A.2 Scenario 2}

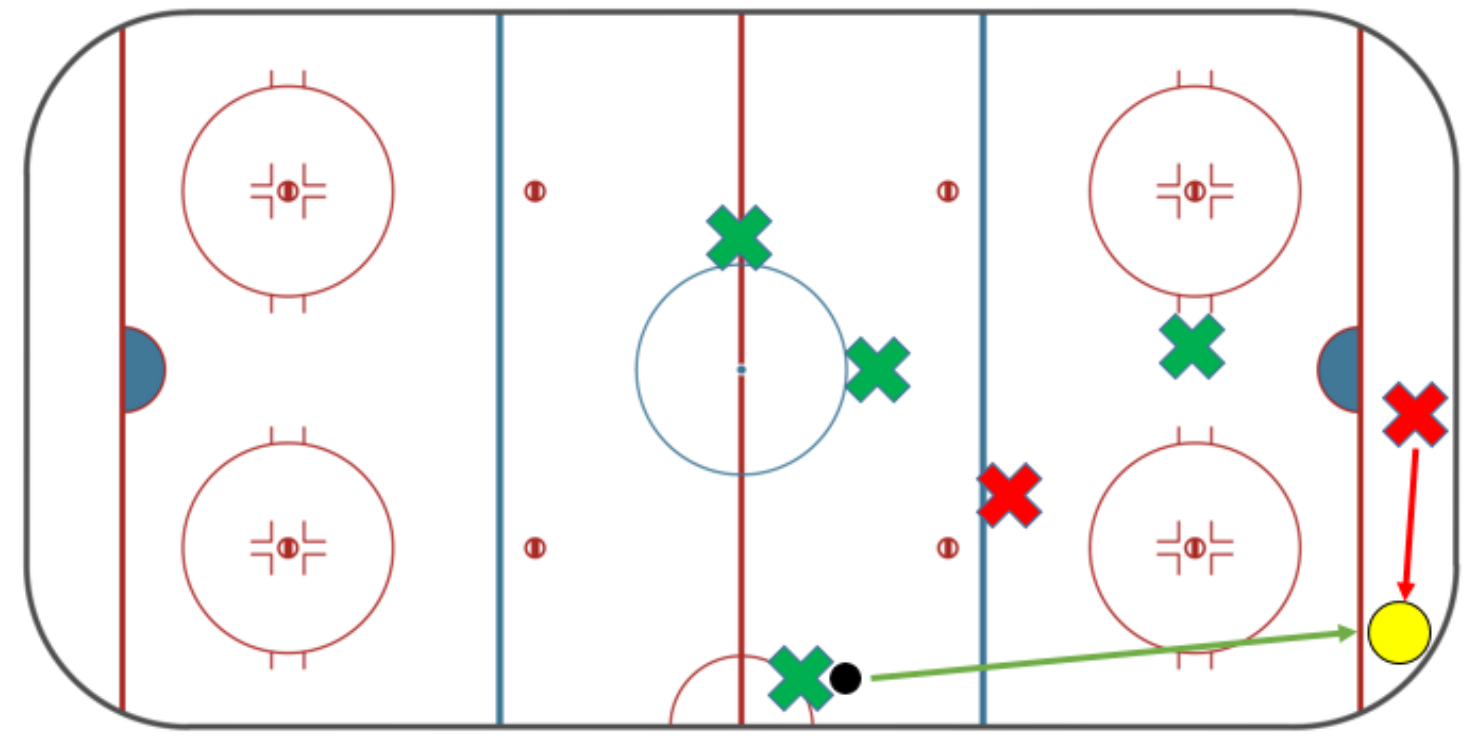

Figure A.5: Scene 5 


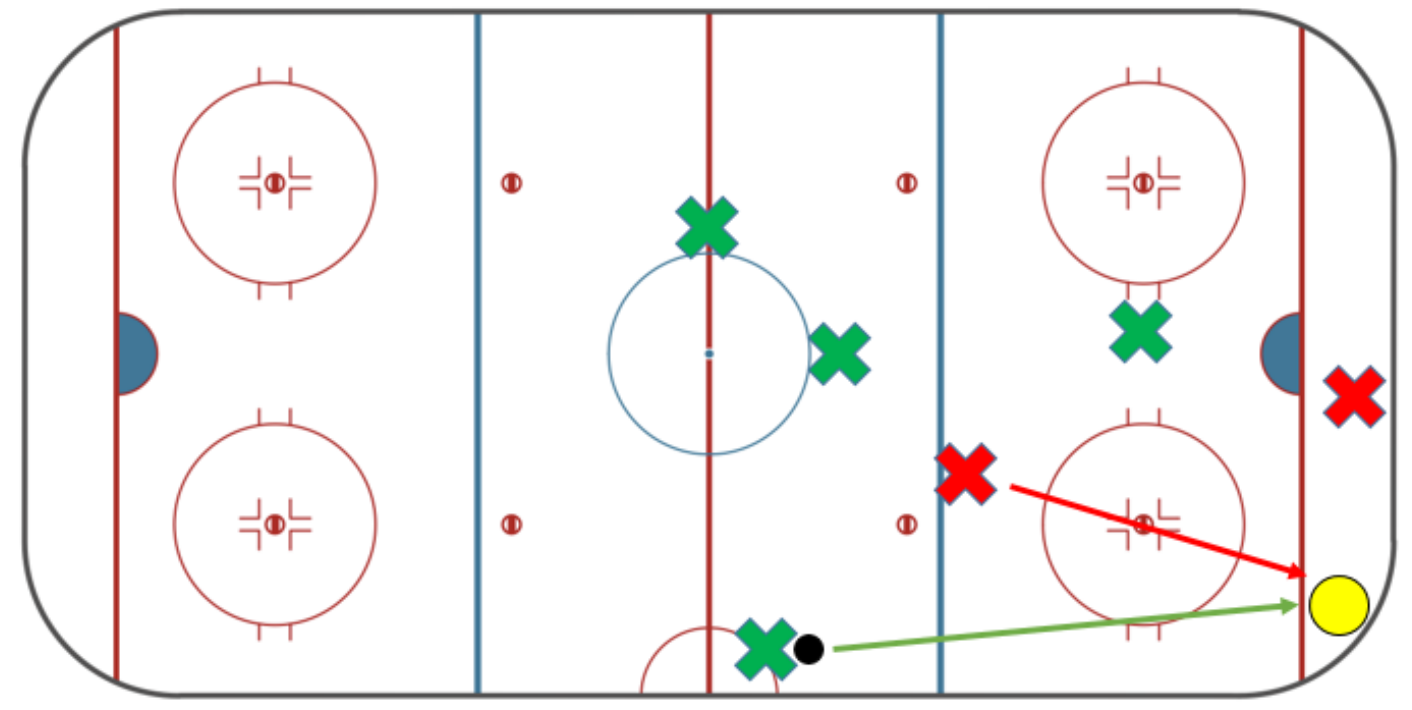

Figure A.6: Scene 6

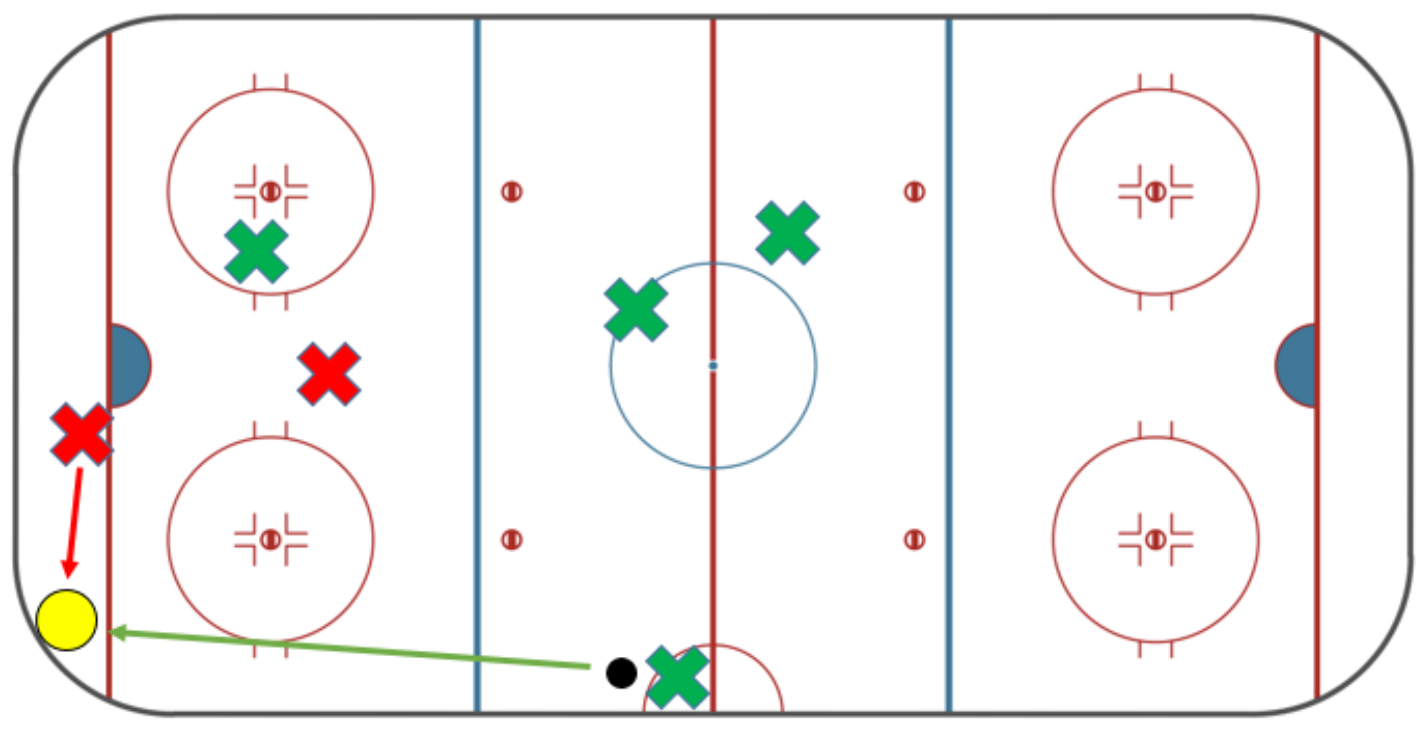

Figure A.7: Scene 7 


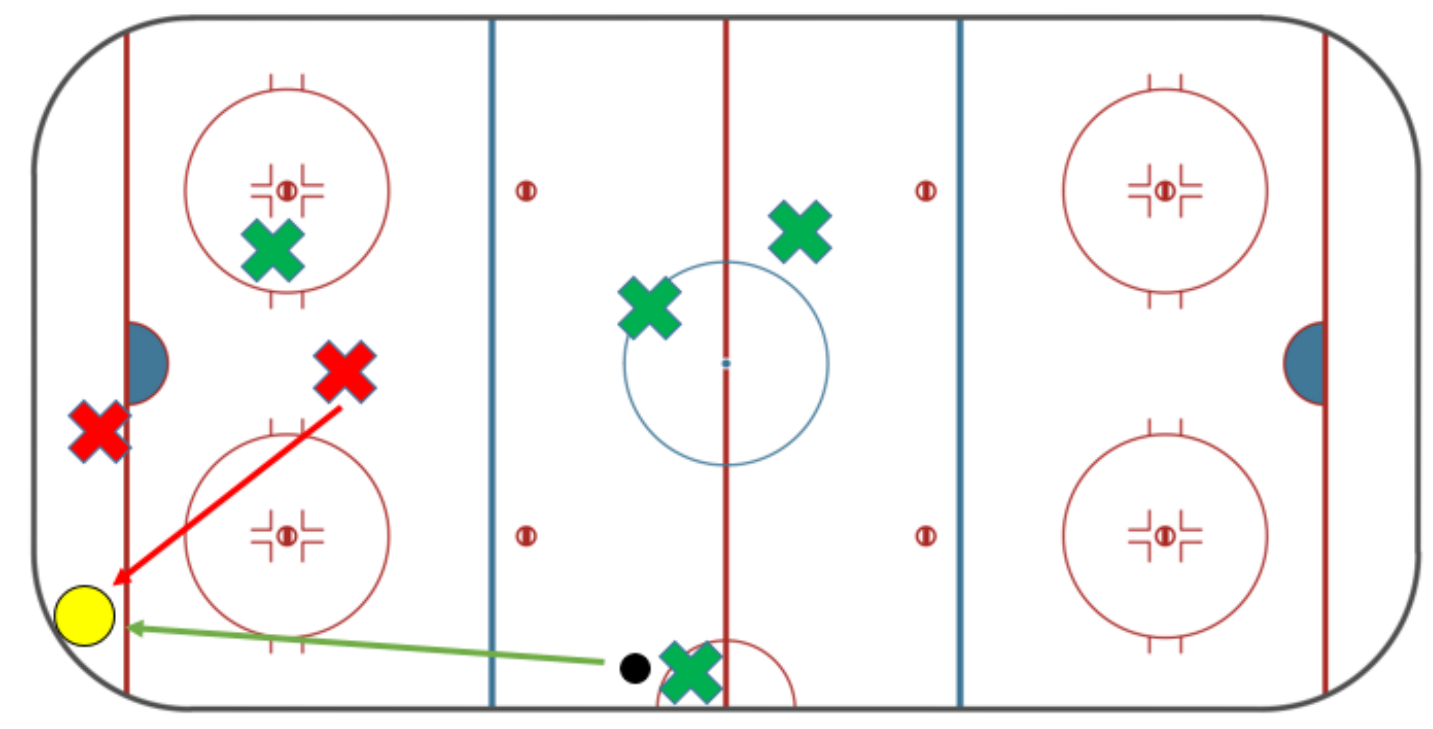

Figure A.8: Scene 8

\section{A.3 Scenario 3}

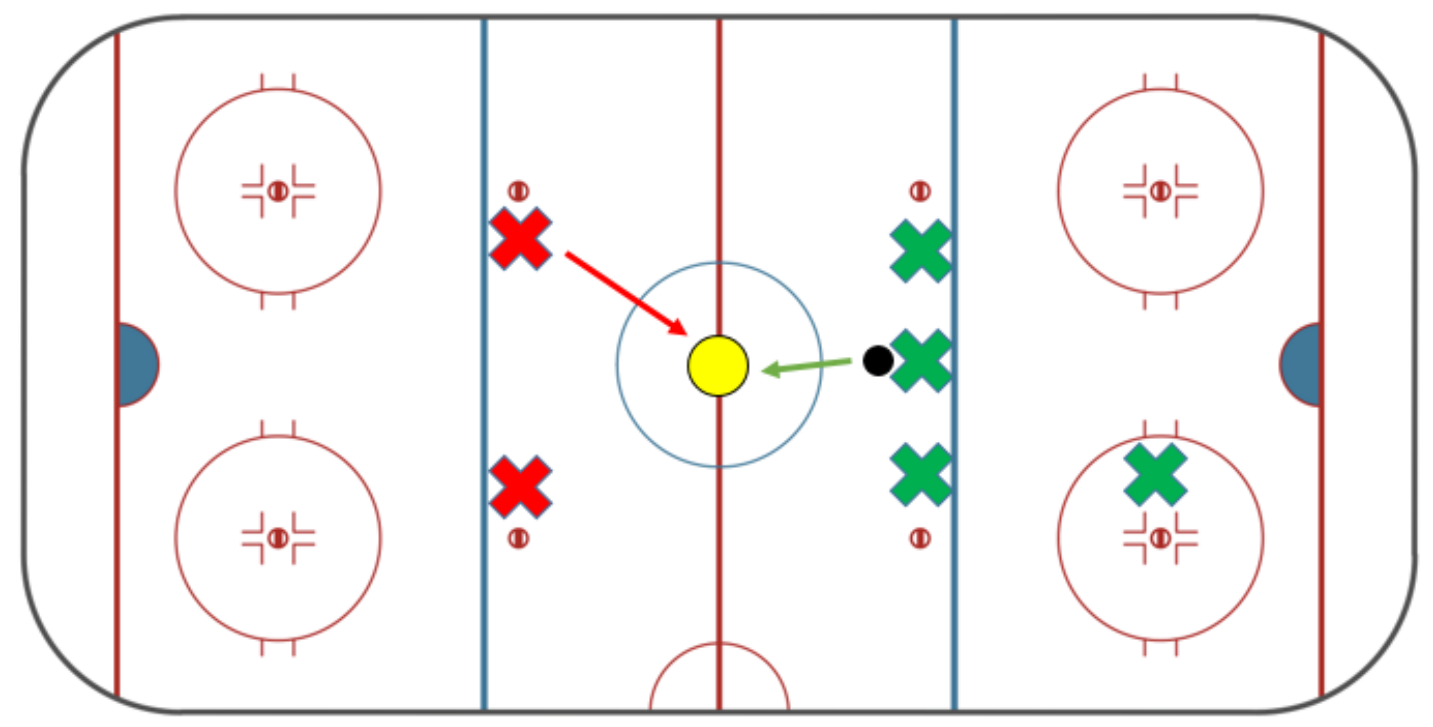

Figure A.9: Scene 9 


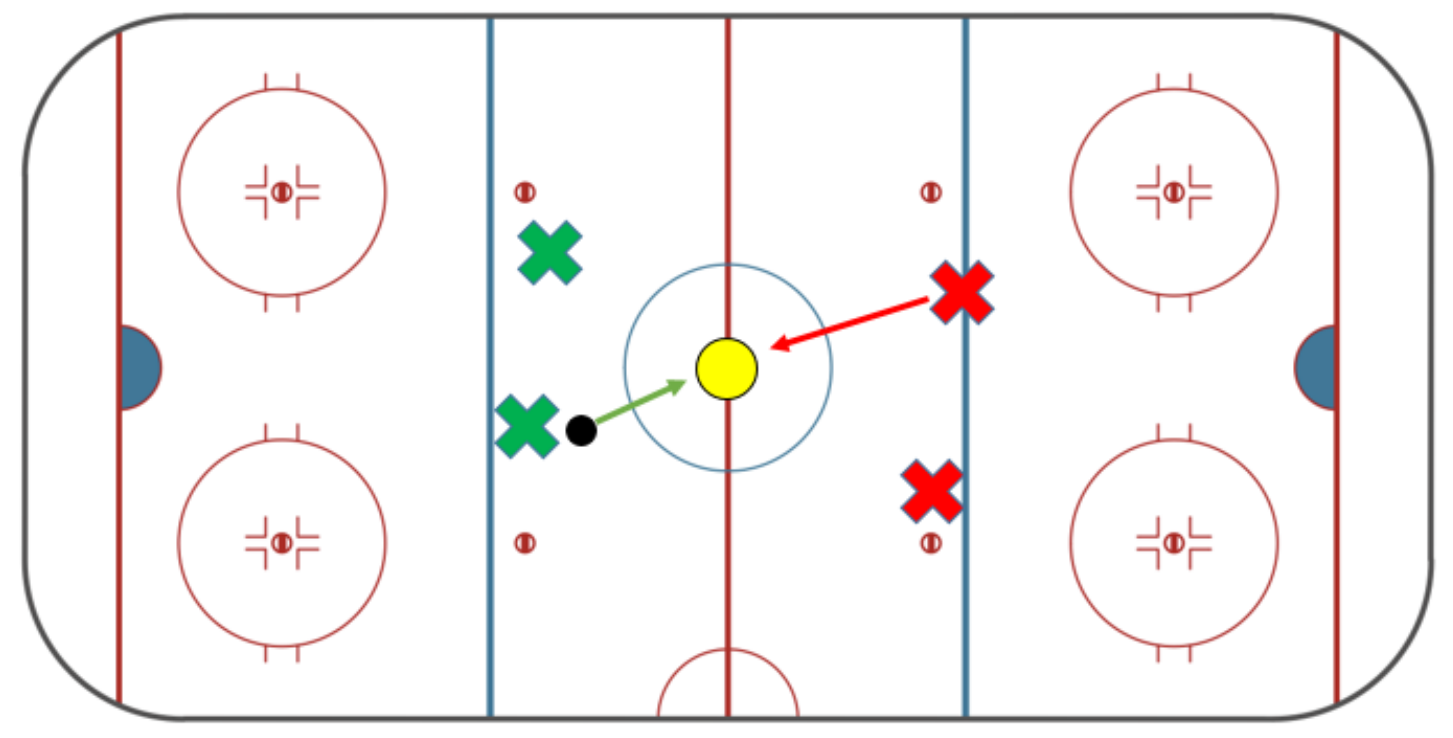

Figure A.10: Scene 10

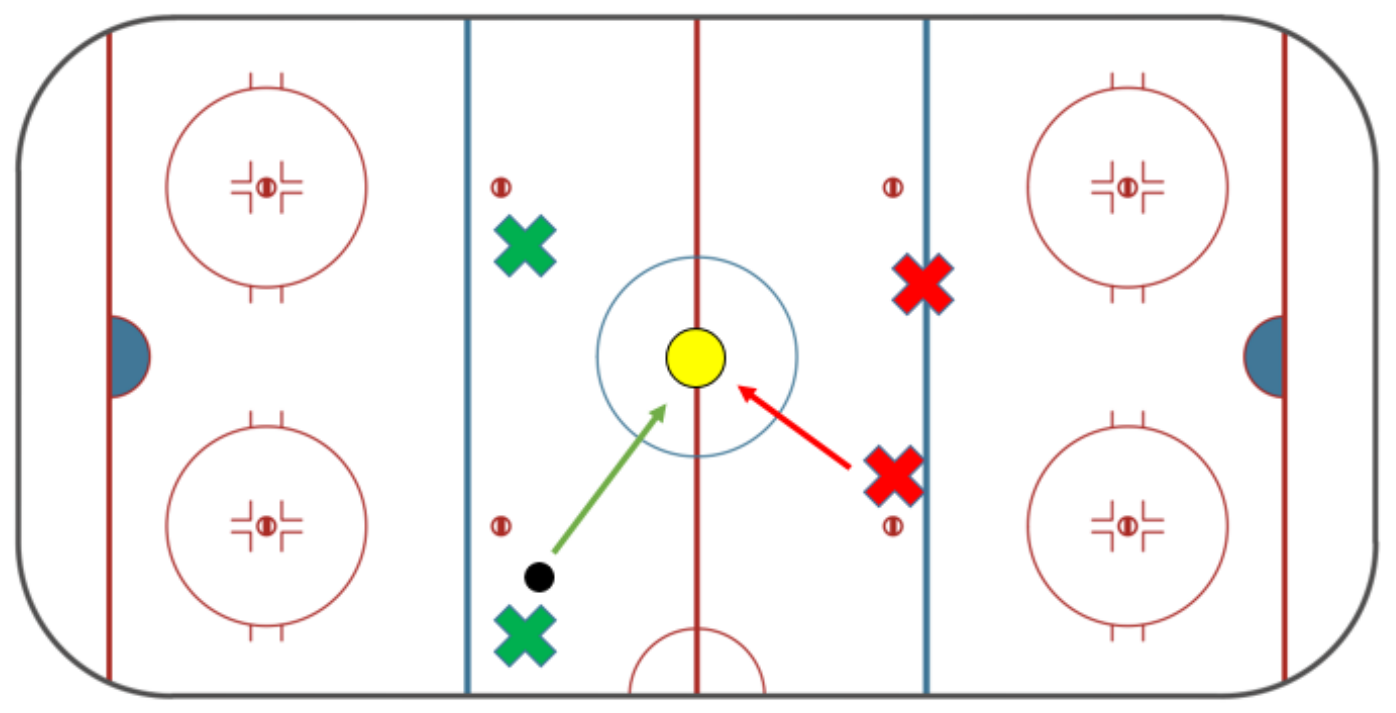

Figure A.11: Scene 11 


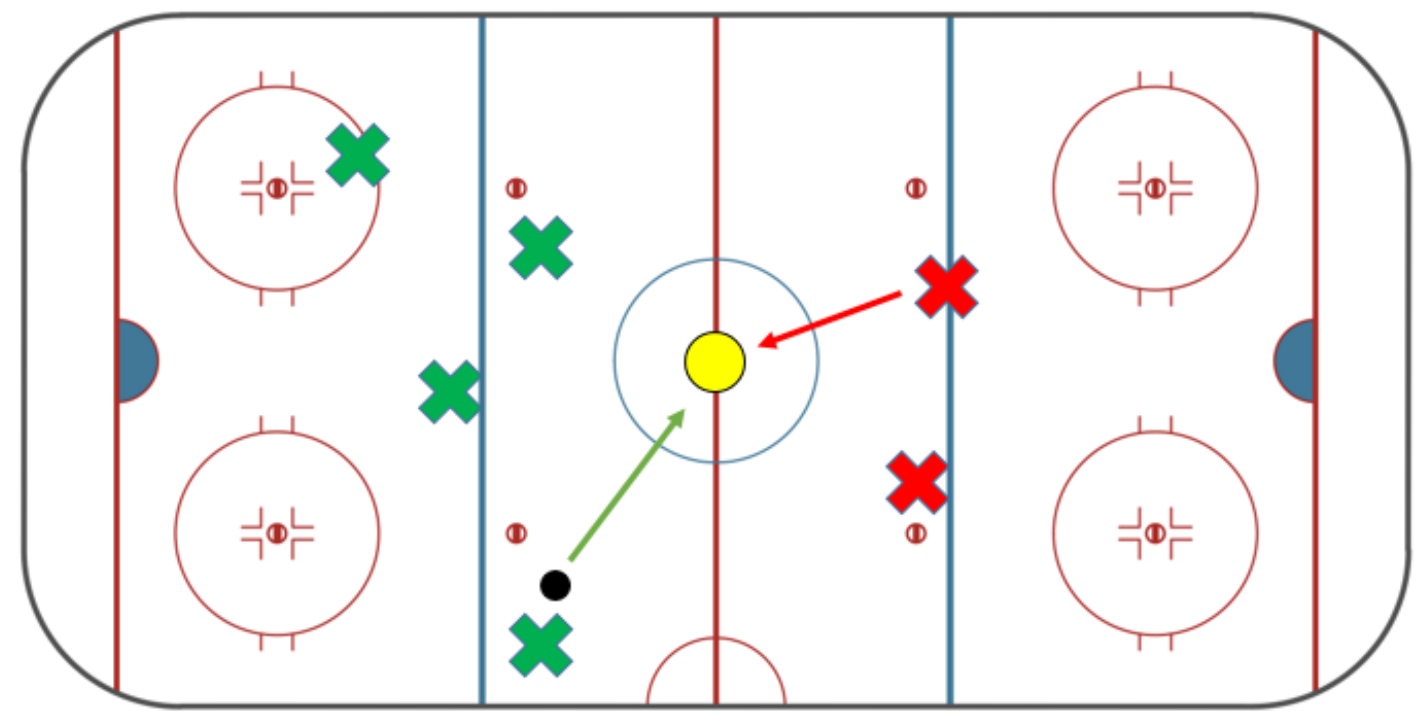

Figure A.12: Scene 12

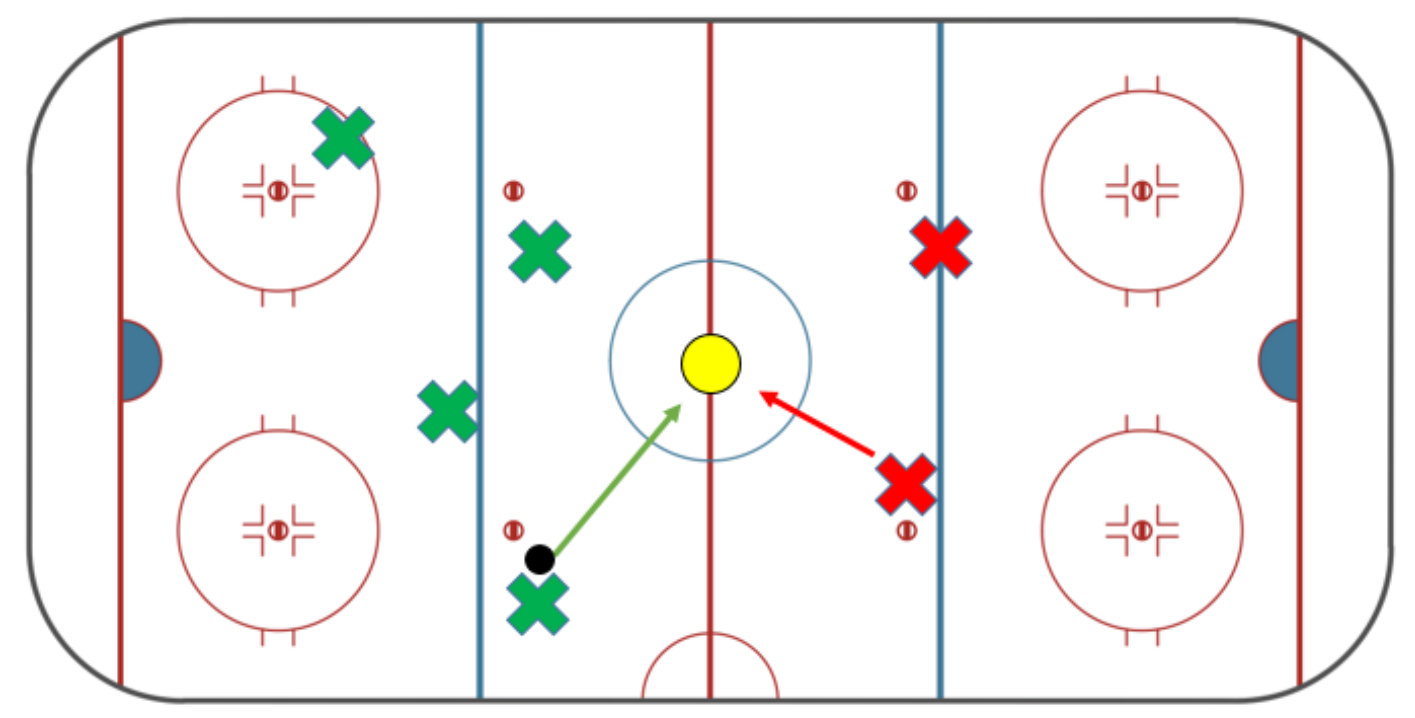

Figure A.13: Scene 13 


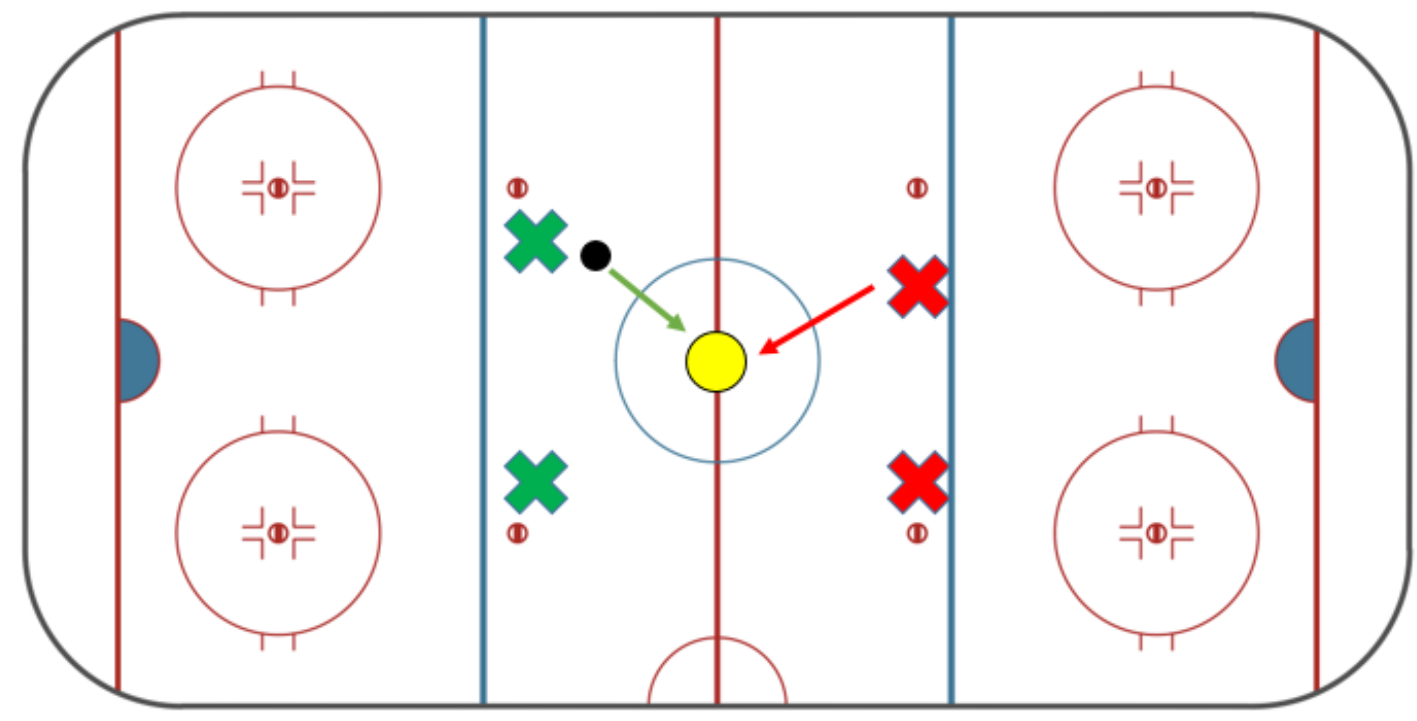

Figure A.14: Scene 14

\section{A.4 Scenario 4}

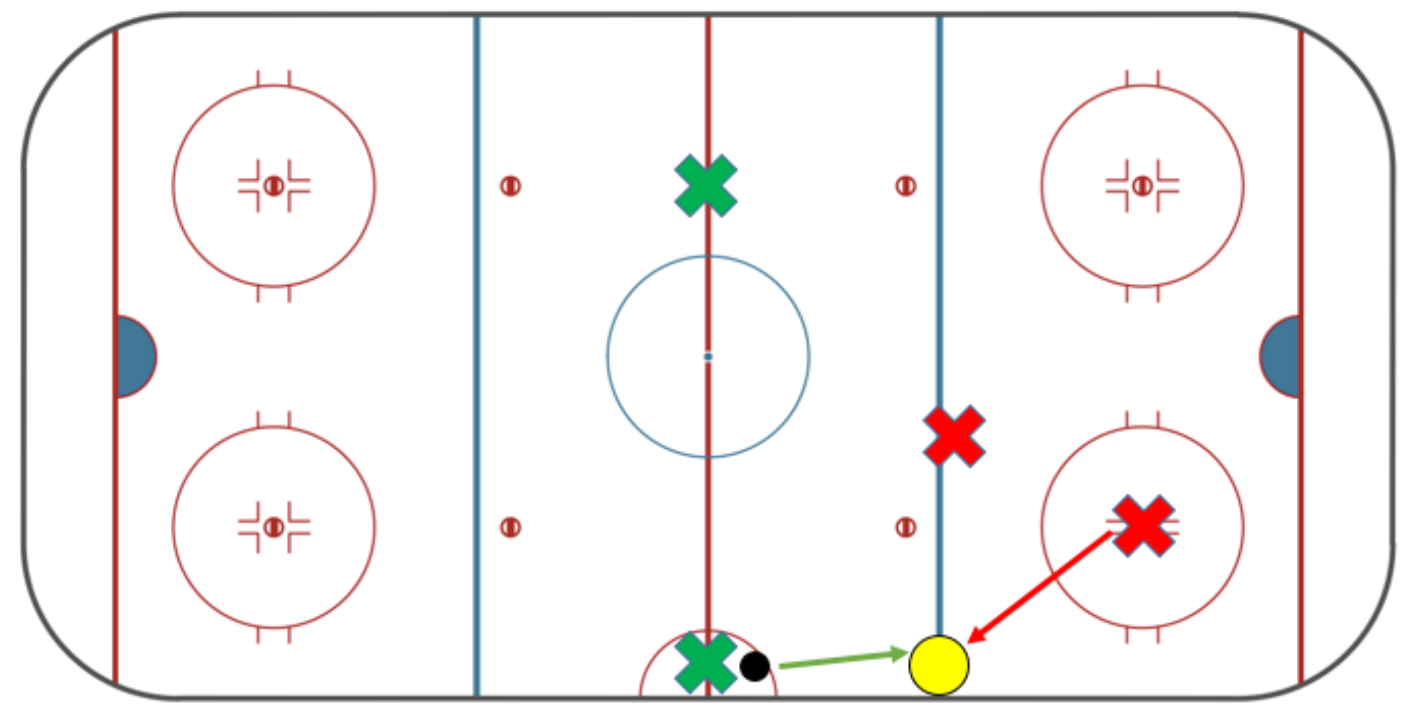

Figure A.15: Scene 15 


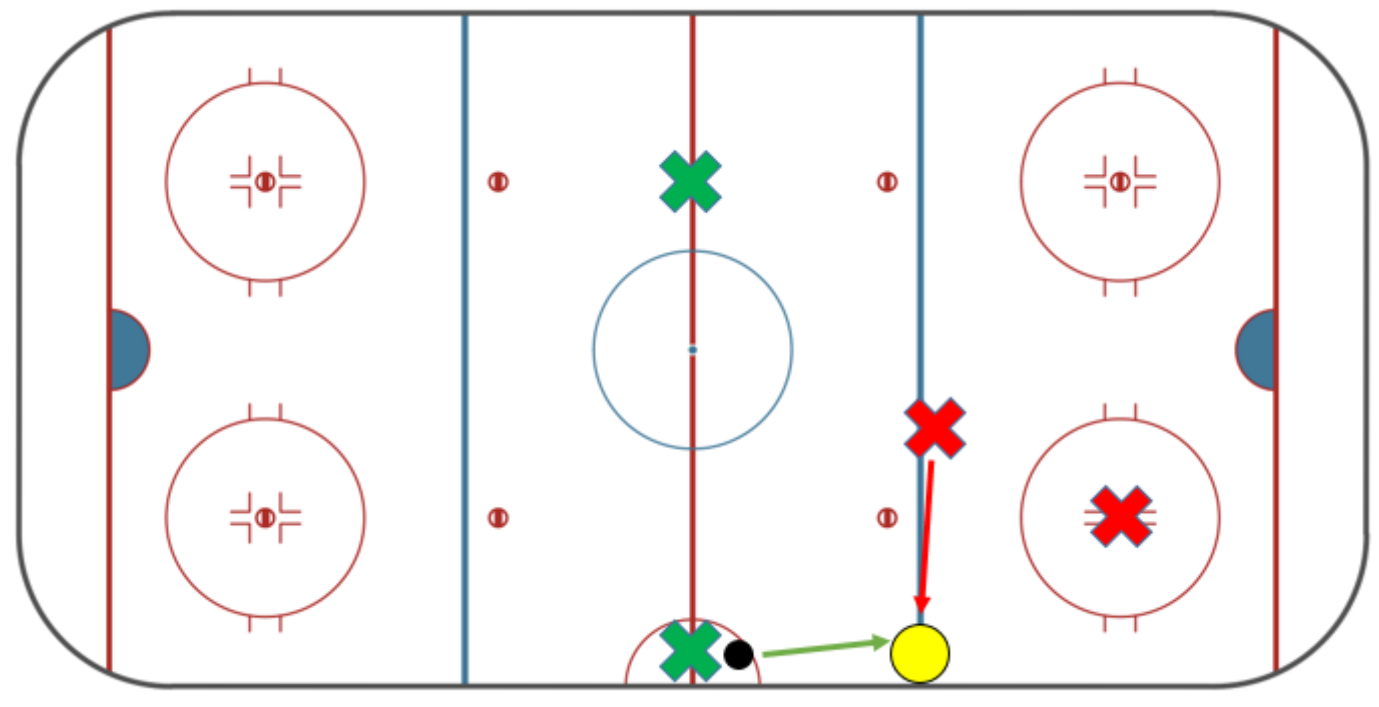

Figure A.16: Scene 16

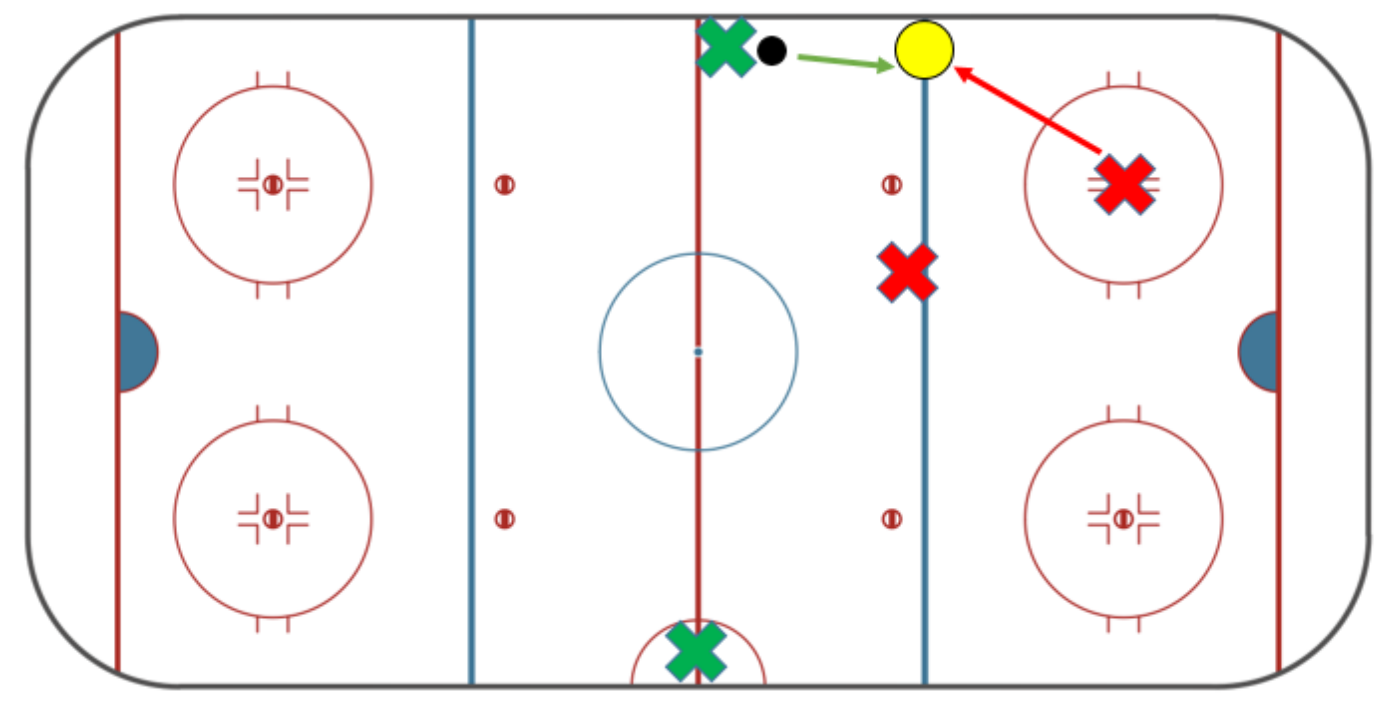

Figure A.17: Scene 17 


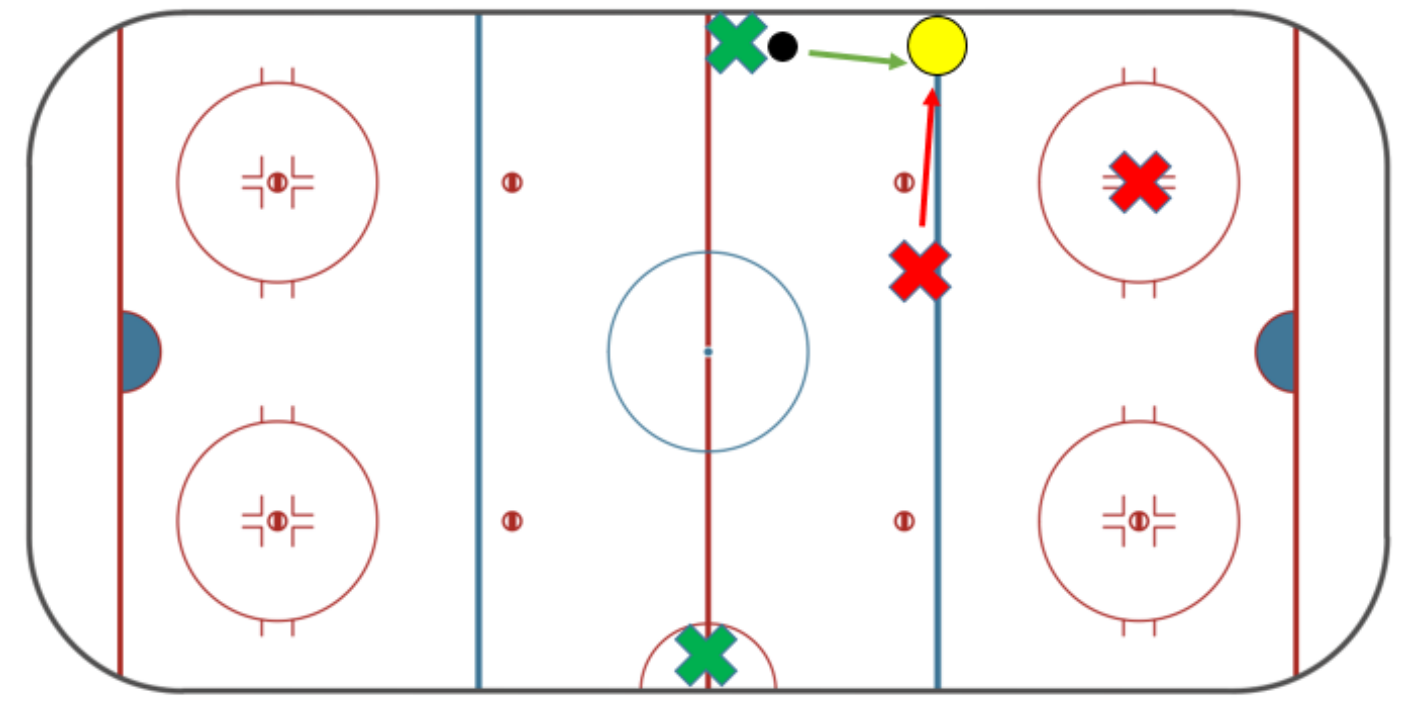

Figure A.18: Scene 18

\section{A.5 Scenario 5}



Figure A.19: Scene 19 


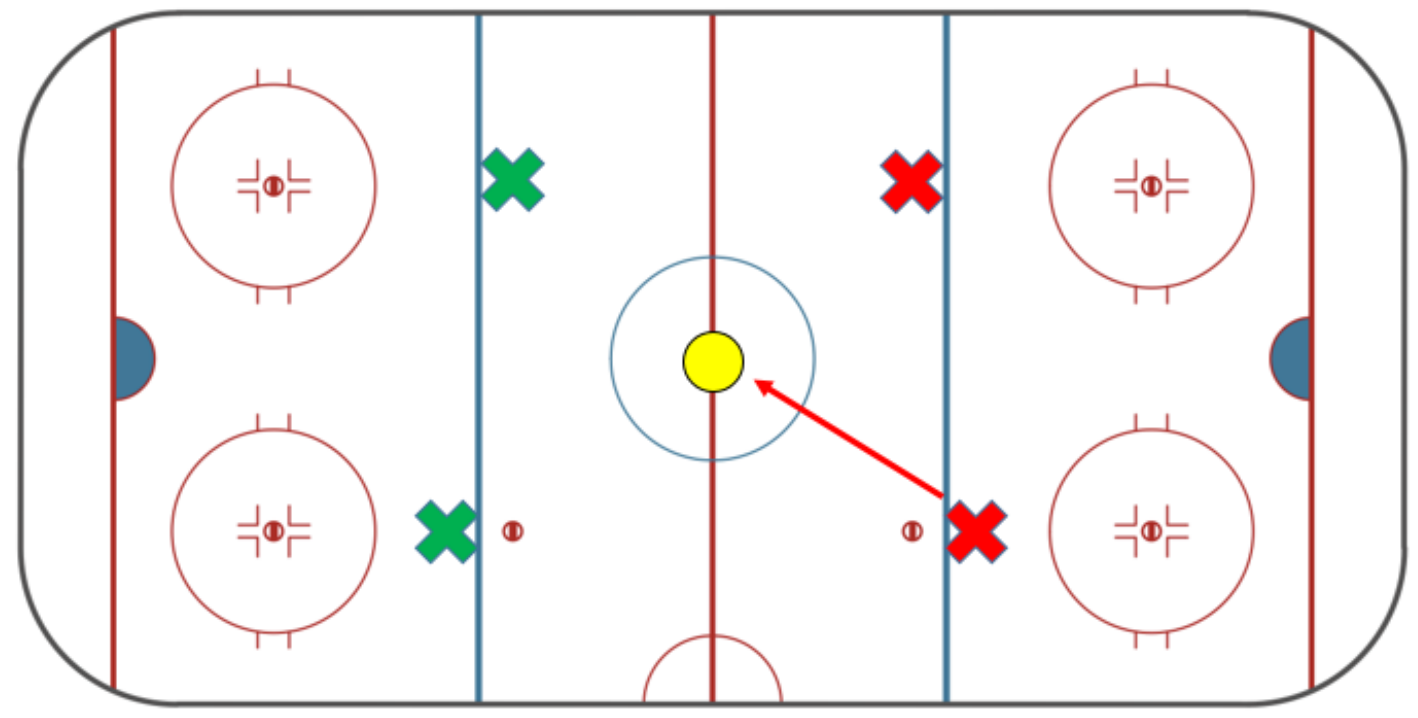

Figure A.20: Scene 20

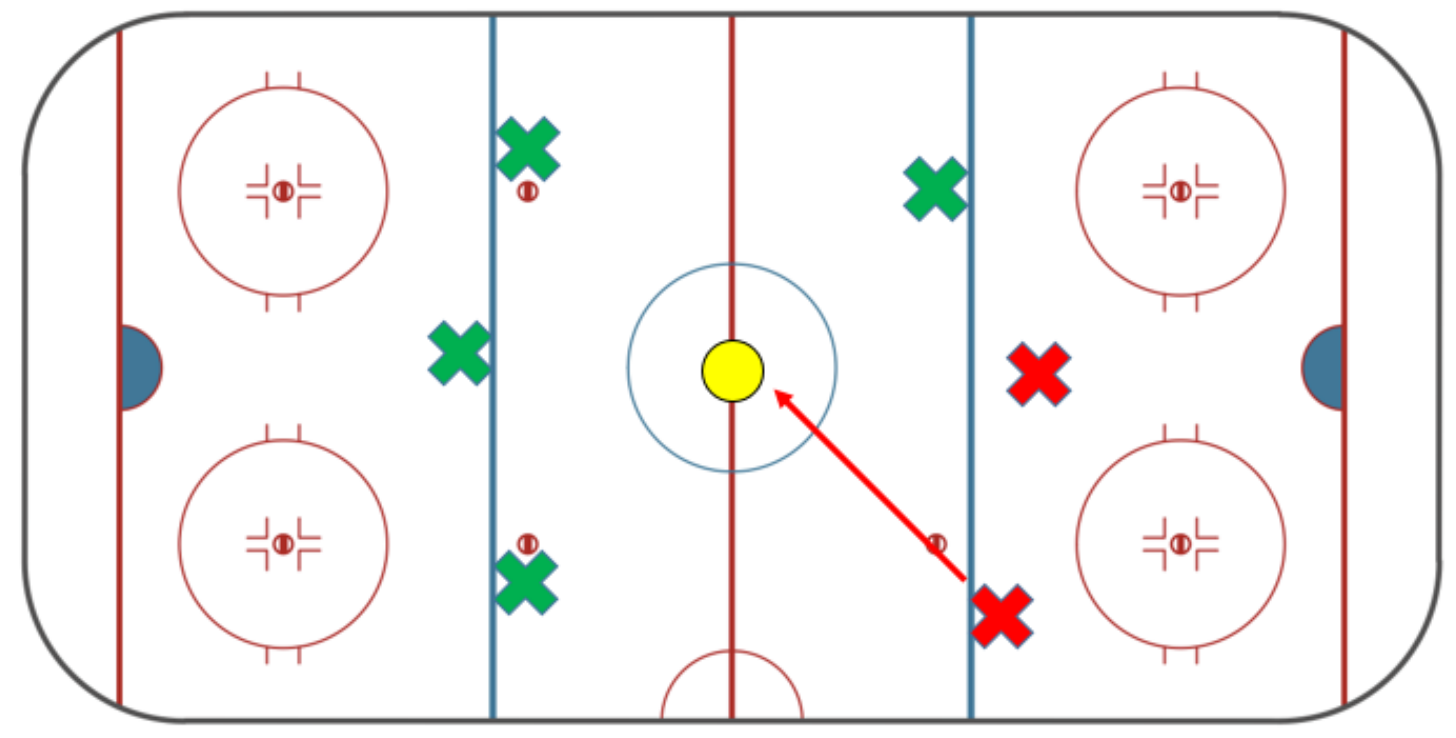

Figure A.21: Scene 21 


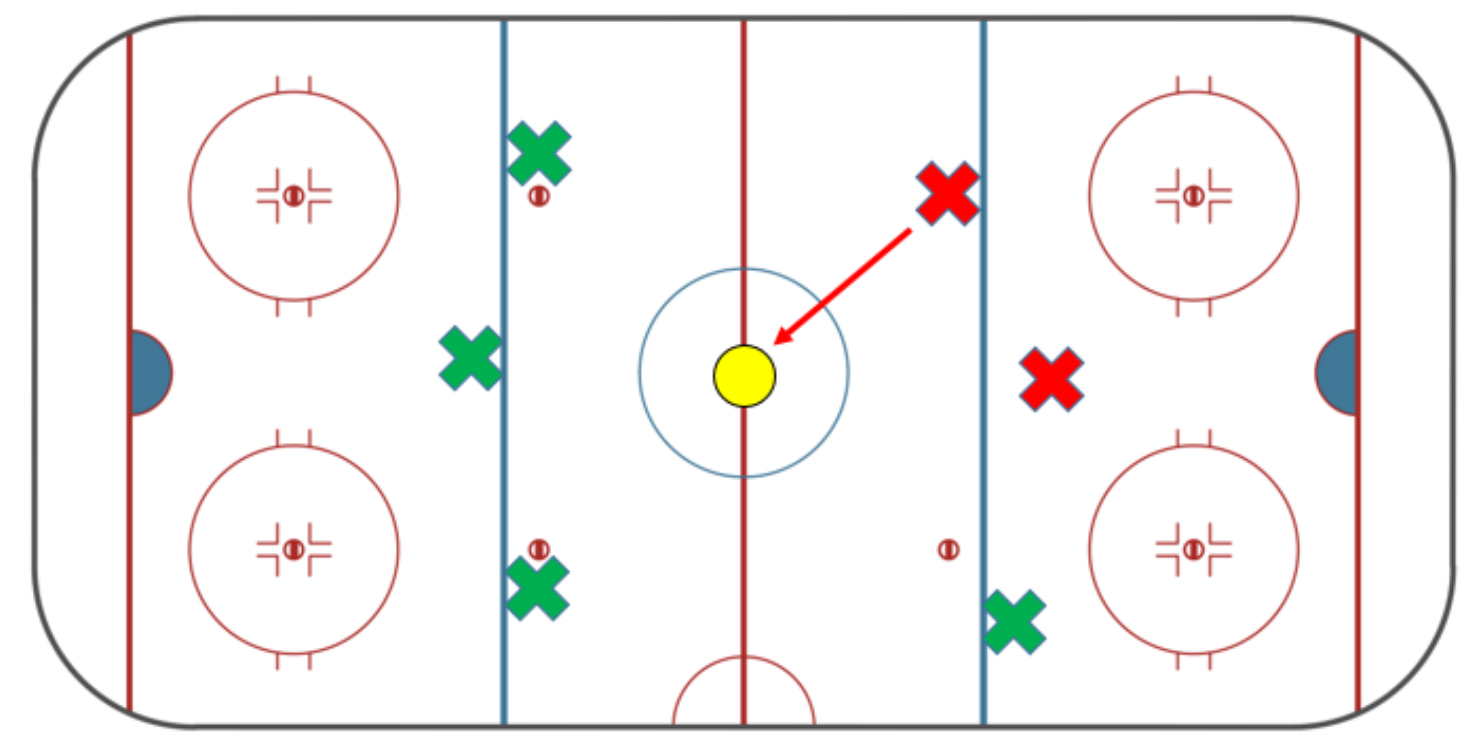

Figure A.22: Scene 22

\section{A.6 Scenario 6}

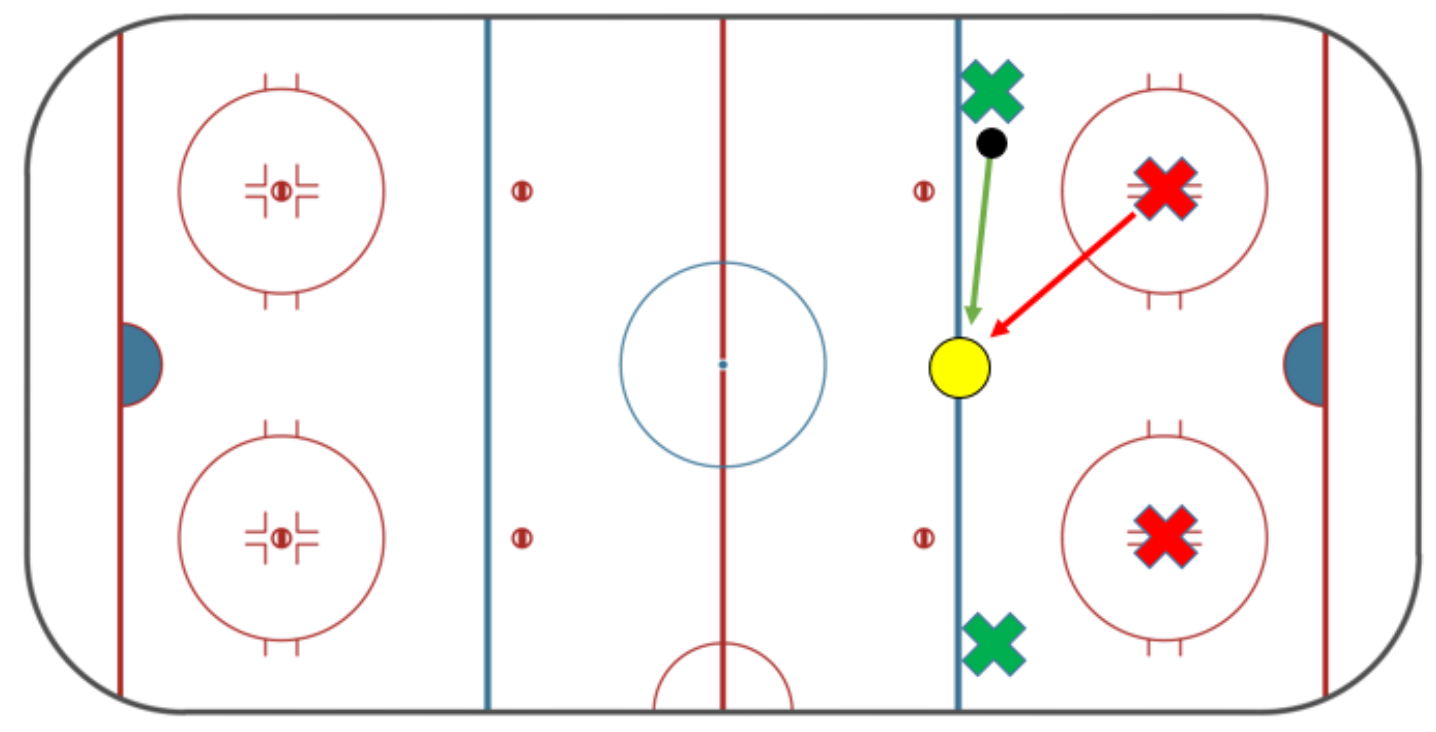

Figure A.23: Scene 23 


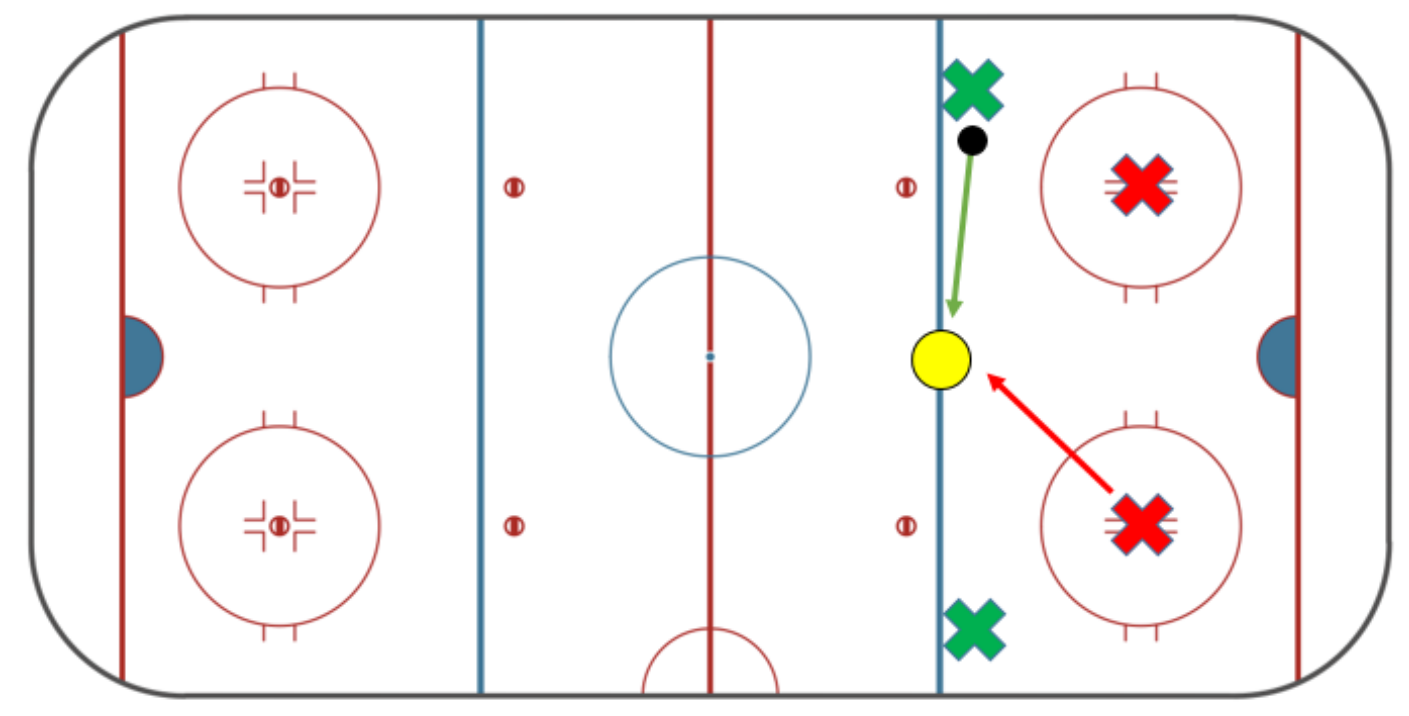

Figure A.24: Scene 24

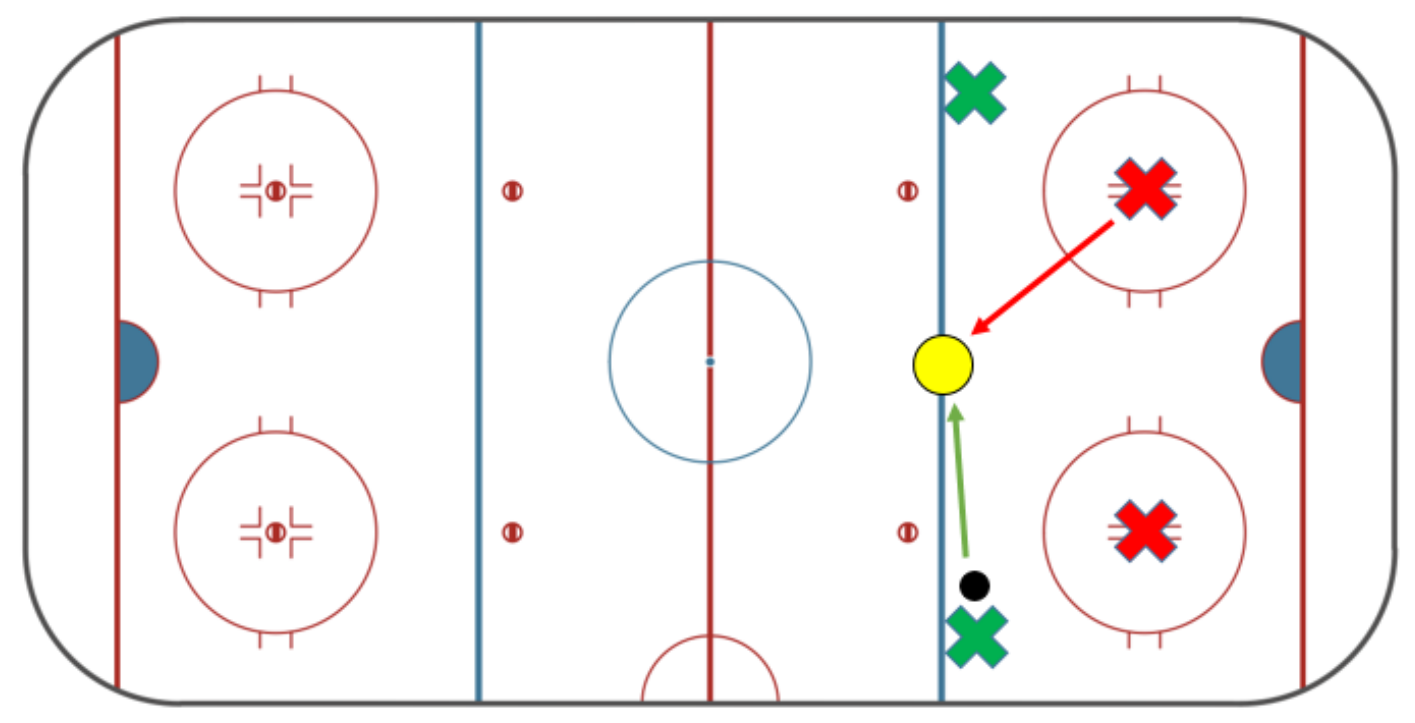

Figure A.25: Scene 25 


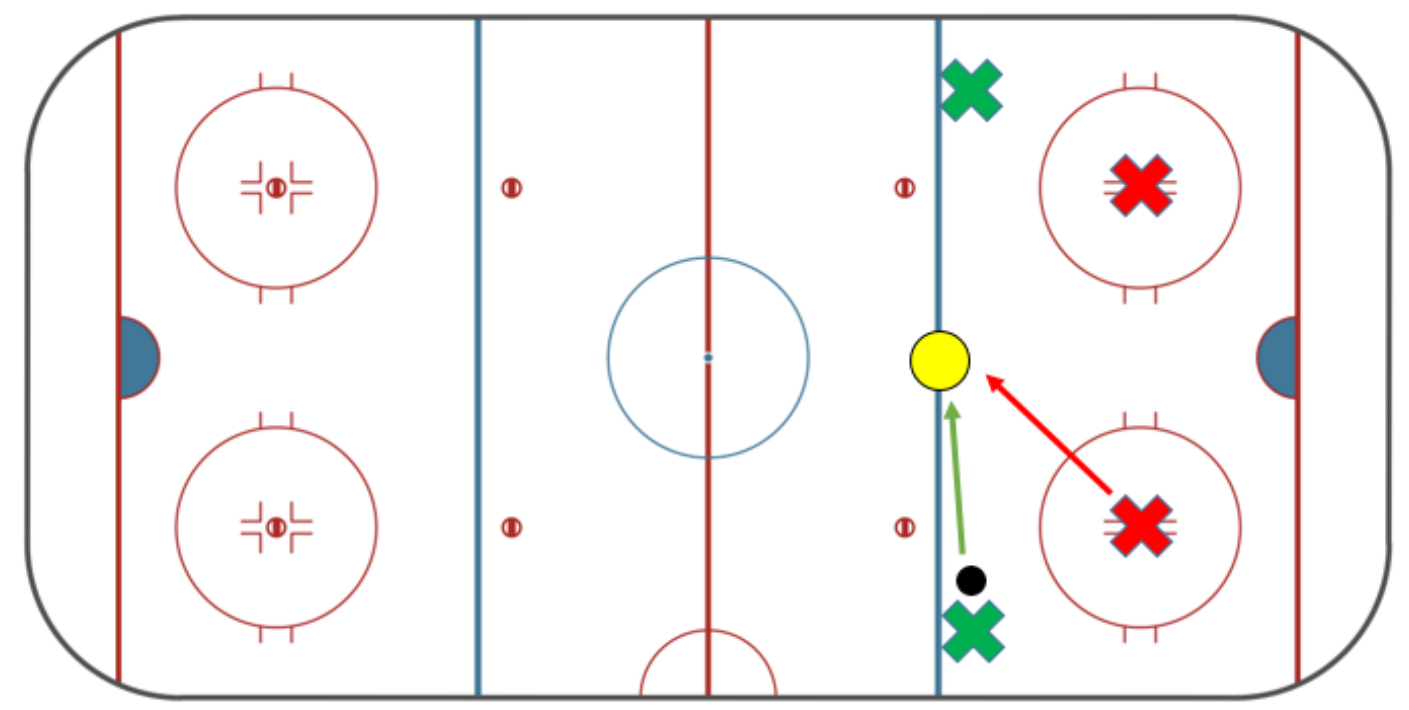

Figure A.26: Scene 26

\section{A.7 Scenario 7}

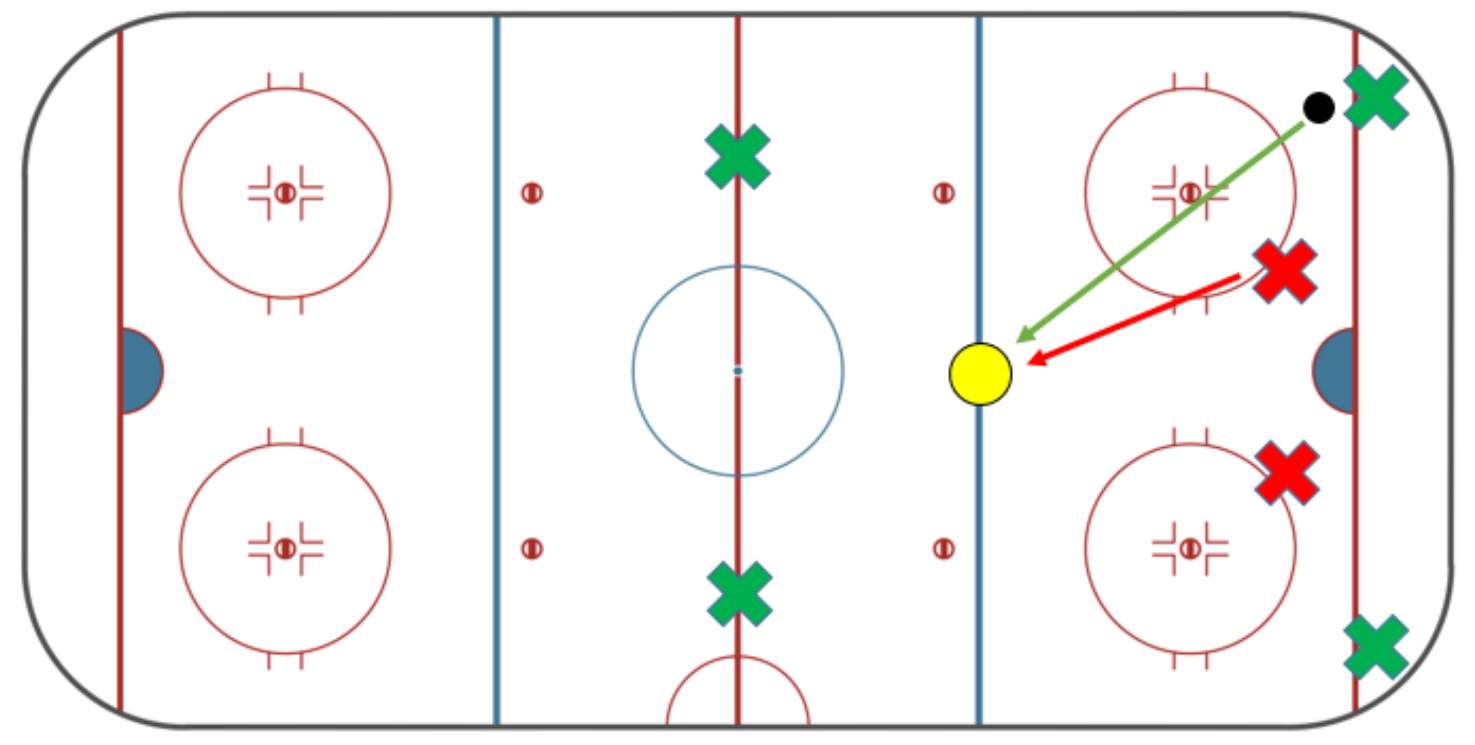

Figure A.27: Scene 27 


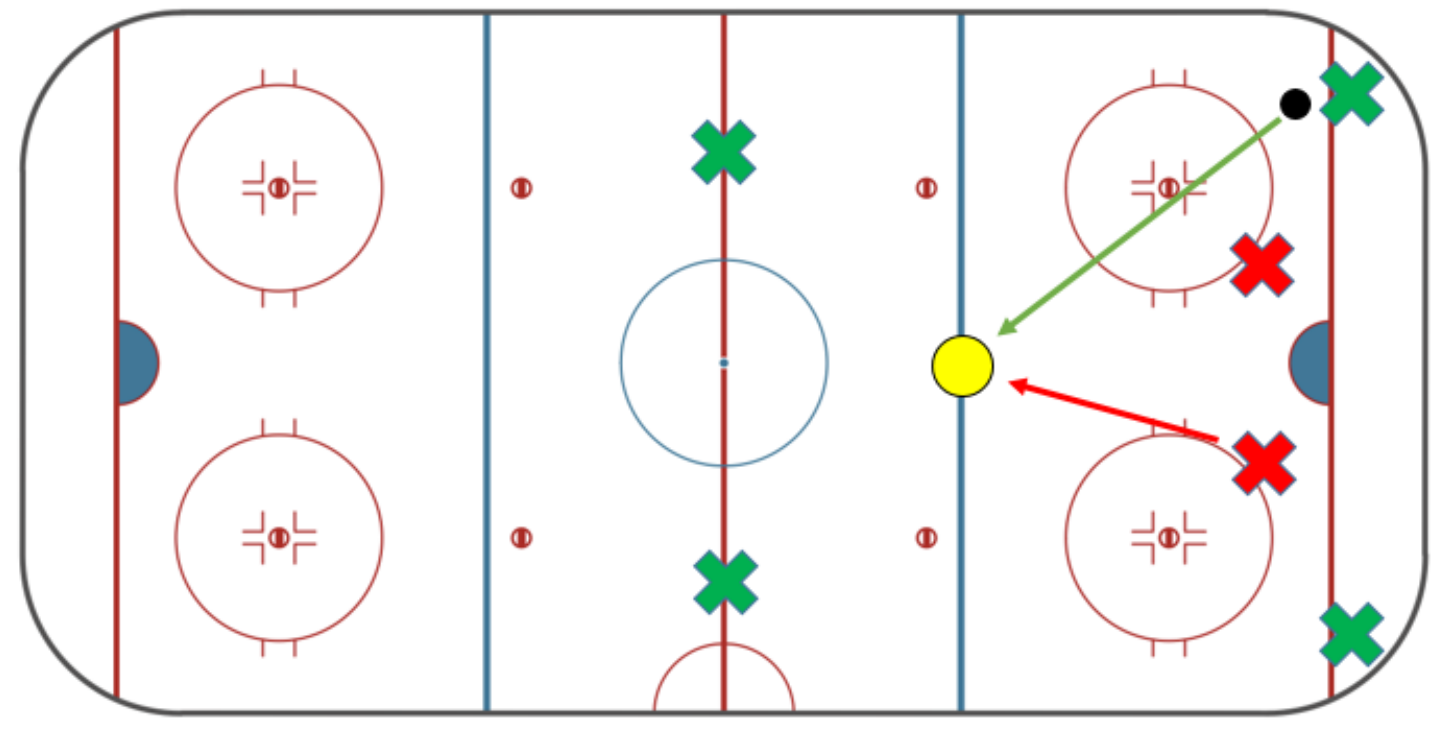

Figure A.28: Scene 28

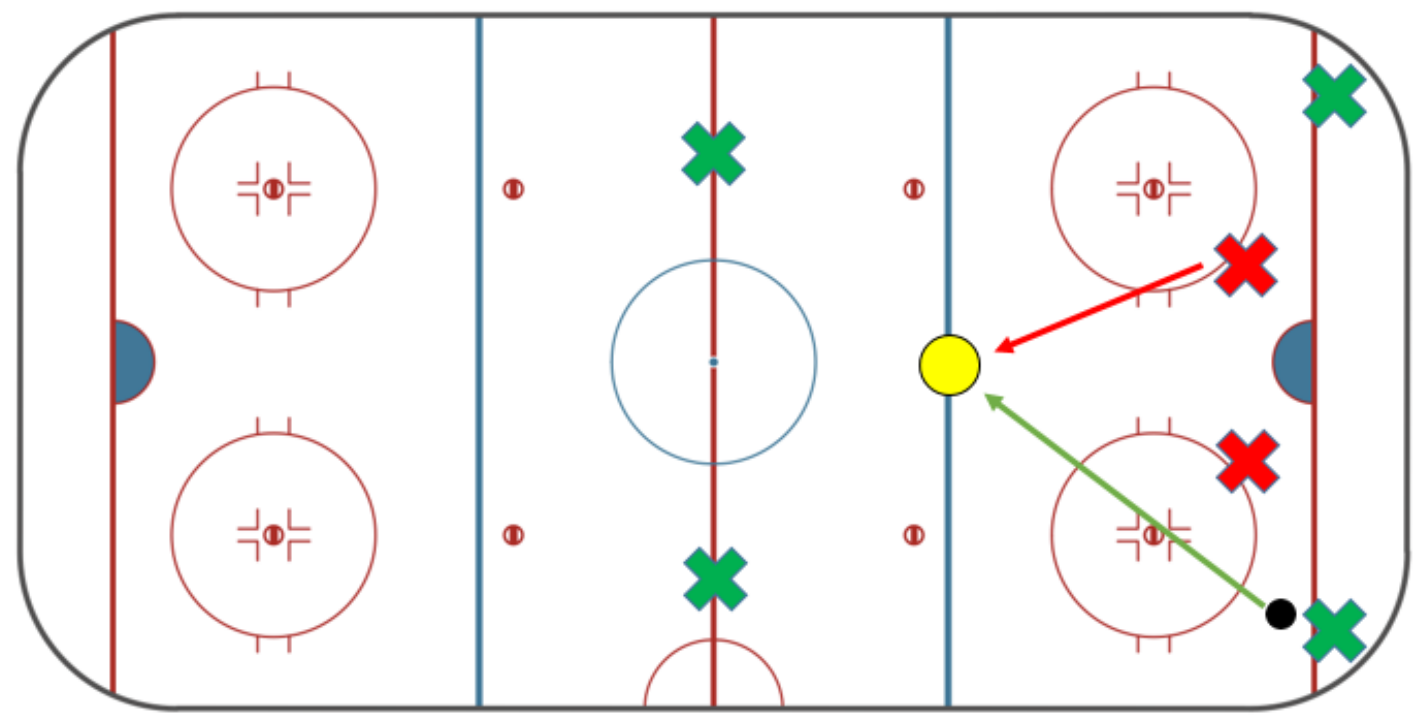

Figure A.29: Scene 29 


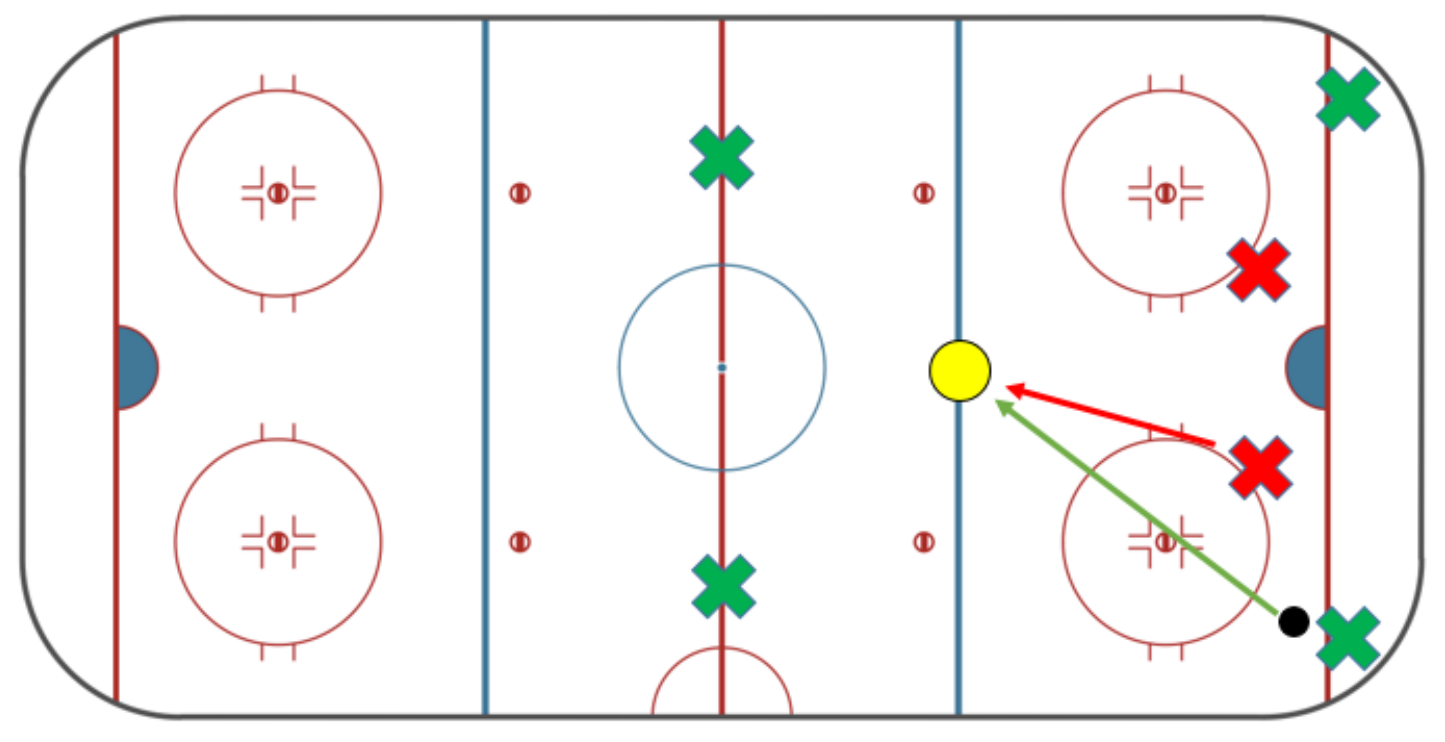

Figure A.30: Scene 30 


\section{Appendix B}

\section{Survey Questions and Ethics Documents}

The sections below show the surveys and data collection form that were filled out by each participant. 


\title{
B.1 SCAT Survey
}

\author{
How do you feel?
}

You should score yourself on the following symptoms based on how you feel now

Headache

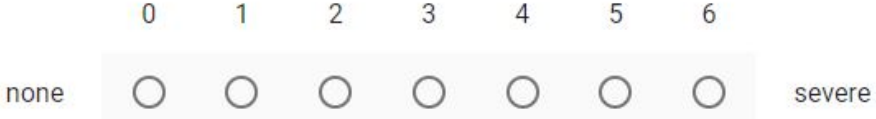

"Pressure in head"

$\begin{array}{lllllllll} & 0 & 1 & 2 & 3 & 4 & 5 & 6 & \\ \text { none } & \bigcirc & \bigcirc & \bigcirc & \bigcirc & \bigcirc & \bigcirc & \bigcirc & \text { severe }\end{array}$

Neck pain

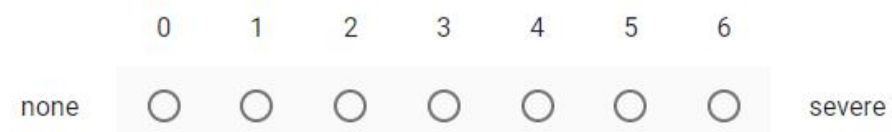

Nausea or vomiting

$\begin{array}{lllllllll} & 0 & 1 & 2 & 3 & 4 & 5 & 6 & \\ \text { none } & \bigcirc & \bigcirc & \bigcirc & \bigcirc & \bigcirc & \bigcirc & \bigcirc & \text { severe }\end{array}$

Dizziness

$\begin{array}{lllllllll} & 0 & 1 & 2 & 3 & 4 & 5 & 6 & \\ \text { none } & \bigcirc & \bigcirc & \bigcirc & \bigcirc & \bigcirc & \bigcirc & \bigcirc & \text { severe }\end{array}$




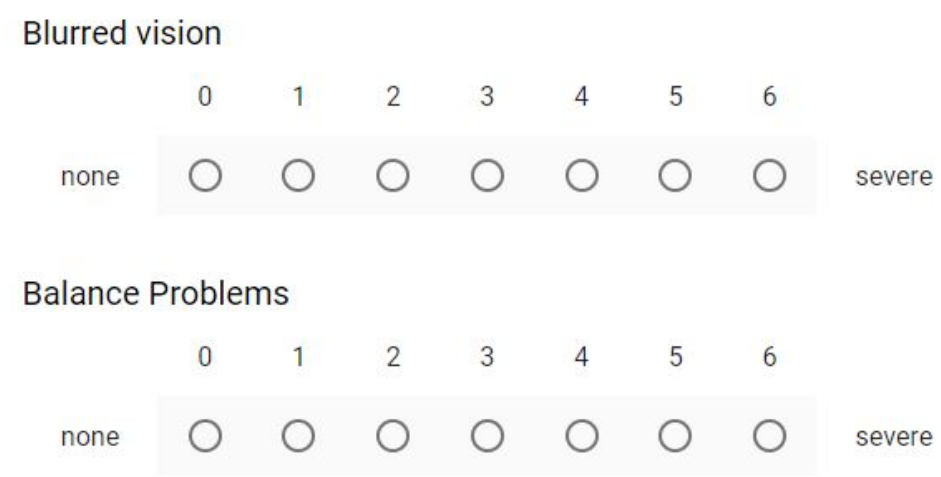

Sensitivity to light

$\begin{array}{lllllllll} & 0 & 1 & 2 & 3 & 4 & 5 & 6 & \\ \text { none } & \bigcirc & \bigcirc & \bigcirc & \bigcirc & \bigcirc & \bigcirc & \bigcirc & \text { severe }\end{array}$

Sensitivity to noise

$\begin{array}{lllllllll} & 0 & 1 & 2 & 3 & 4 & 5 & 6 & \\ \text { none } & \bigcirc & \bigcirc & \bigcirc & \bigcirc & \bigcirc & \bigcirc & \bigcirc & \text { severe }\end{array}$

Feeling slowed down

$\begin{array}{lllllllll} & 0 & 1 & 2 & 3 & 4 & 5 & 6 & \\ \text { none } & \bigcirc & \bigcirc & \bigcirc & \bigcirc & \bigcirc & \bigcirc & \bigcirc & \text { severe }\end{array}$

Feeling like "in a fog"

$\begin{array}{lllllllll} & 0 & 1 & 2 & 3 & 4 & 5 & 6 & \\ \text { none } & \bigcirc & \bigcirc & \bigcirc & \bigcirc & \bigcirc & \bigcirc & \bigcirc & \text { severe }\end{array}$


"Don't feel right"

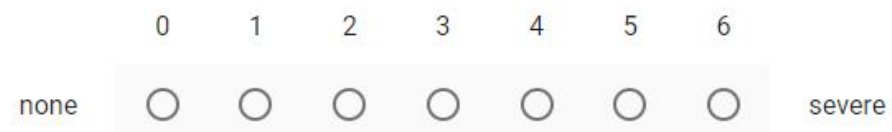

Difficulty concentrating

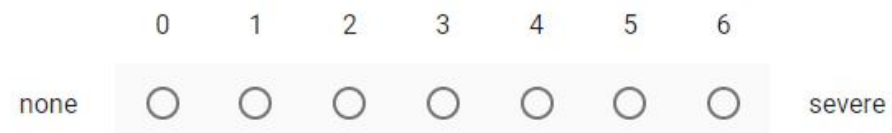

Difficulty remembering

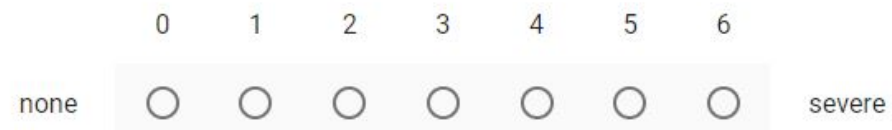

Fatigue or low energy

$\begin{array}{lllllllll} & 0 & 1 & 2 & 3 & 4 & 5 & 6 & \\ \text { none } & \bigcirc & \bigcirc & \bigcirc & \bigcirc & \bigcirc & \bigcirc & \bigcirc & \text { severe }\end{array}$

Confusion

$\begin{array}{lllllllll} & 0 & 1 & 2 & 3 & 4 & 5 & 6 & \\ \text { none } & \bigcirc & \bigcirc & \bigcirc & \bigcirc & \bigcirc & \bigcirc & \bigcirc & \text { severe }\end{array}$

Drowsiness

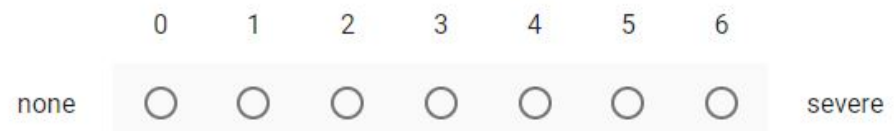


Trouble falling asleep

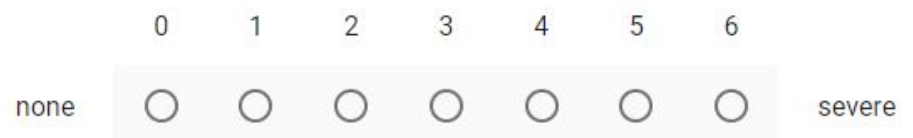

More emotional

$\begin{array}{lllllllll} & 0 & 1 & 2 & 3 & 4 & 5 & 6 & \\ \text { none } & \bigcirc & \bigcirc & \bigcirc & \bigcirc & \bigcirc & \bigcirc & \bigcirc & \text { severe }\end{array}$

Irritability

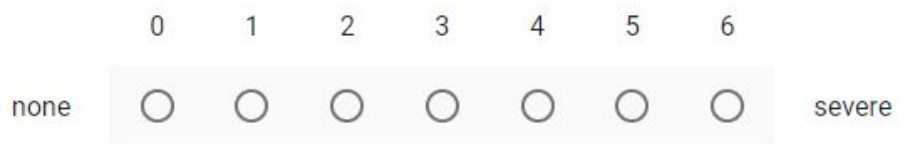

Sadness

$\begin{array}{lllllll}0 & 1 & 2 & 3 & 4 & 5 & 6\end{array}$

none $\bigcirc \bigcirc \bigcirc \bigcirc \bigcirc \bigcirc \bigcirc$ severe

Nervous or Anxious

\begin{tabular}{|c|c|c|c|c|c|c|c|}
\hline & 0 & 1 & 2 & 3 & 4 & 5 & 6 \\
\hline one & 0 & 0 & 0 & 0 & 0 & 0 & 0 \\
\hline
\end{tabular}

Do the symptoms get worse with physical activity?

$\bigcirc$ Yes

No 


\section{B.2 ACE Survey}

\section{Acute Concussion Evaluation (ACE)}

\section{B. Symptom Check List}

Have you experienced any of these symptoms any more than usual today? Indicate presence of each symptom $(0=\mathrm{No}, 1=\mathrm{Yes})$.

\section{Physical (10)}

Headache

$\bigcirc 0$

$\bigcirc 1$

Nausea

$\bigcirc 0$

$\bigcirc 1$

Vomiting

$\bigcirc 0$

$\bigcirc 1$

Balance Problems

$\bigcirc 0$

$\bigcirc 1$ 
Dizziness

$\bigcirc 0$

○ 1

Visual Problems

$\bigcirc 0$

○ 1

Fatigue

00

○ 1

Sensitivity to light

$\bigcirc 0$

○ 1

Sensitivity to noise

O 0

$\bigcirc 1$

Numbness/Tingling

$\bigcirc 0$

○ 1 


\section{Cognitive (4)}

Feeling mentally foggy

$\bigcirc 0$

O 1

Feeling slowed down

$\bigcirc 0$

○ 1

Difficulty Concentrating

$\bigcirc 0$

○ 1

Difficulty Remembering

$\bigcirc 0$

○ 1 
Emotional (4)

Irritability

$\bigcirc 0$

$\bigcirc 1$

Sadness

$\bigcirc 0$

$\bigcirc 1$

More emotional

$\bigcirc 0$

○ 1

Nervousness

○ 0

○ 1 
Sleep (4)

Drowsiness

○ 0

○ 1

Sleeping less than usual

$\bigcirc 0$

○ 1

Sleeping more than usual

$\bigcirc 0$

○ 1

Trouble falling asleep

$\bigcirc 0$

○ 1 


\section{Exertion}

Do these symptoms worsen with:

Physical Activity

Yes

$\bigcirc$ No

N/A

Cognitive Activity

Yes

No

N/A

\section{Overall Rating}

How different are you acting compared to your normal self?

$\begin{array}{lllllllll} & 0 & 1 & 2 & 3 & 4 & 5 & 6 & \\ \text { Normal } & \bigcirc & \bigcirc & \bigcirc & \bigcirc & \bigcirc & \bigcirc & \bigcirc & \text { Very Different }\end{array}$




\section{Risk Factors for Protracted Recovery}

Concussion History

$\bigcirc$ Yes

$\bigcirc$ No

Previous \# of Concussions

Choose -

Longest Symptom Duration

Your answer

If multiple concussions, less force cause reinjury?

$\bigcirc$ Yes

$\bigcirc$ No

$\mathrm{N} / \mathrm{A}$

When was your last concussion?

Less than 6 months ago

6 months to 1 year ago

1-2 years ago

More than 2 years ago 
Headache History

$\bigcirc$ Yes

$\bigcirc$ No

Prior Treatment for Headache

$\bigcirc$ Yes

$\bigcirc$ No

$\mathrm{N} / \mathrm{A}$

History of Migraine Headaches

$\bigcirc$ Personal

$\bigcirc$ Family

No

\section{Developmental History}

Learning Disabilities

$\bigcirc$ Yes

No

Attention-Deficit/Hyperactivity Disorder

$\bigcirc$ Yes

No

Other Developmental Disorders

Your answer 


\title{
Psychiatric History
}

Anxiety

$\bigcirc$ Yes

$\bigcirc$ No

Depression

$\bigcirc$ Yes

No

Other psychiatric disorders

\section{B.3 Data Collection Form}

\author{
(1). WNIVERSITY OF \\ Data Collection Form \\ Data in this form will not be associated with your name and is used purely to quantify the \\ participants involved. This information will be used collectively as part of a population \\ sample and will not be published on its own.

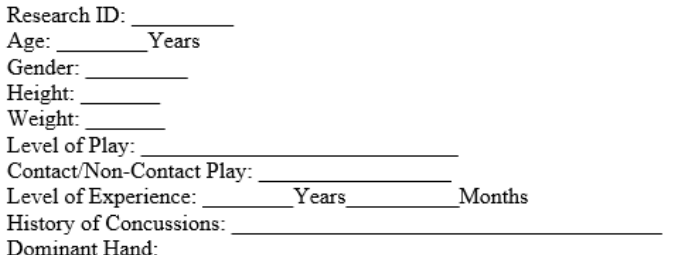

